\title{
GEOMETRY OF THE FANO THREEFOLD OF DEGRE 10 OF THE FIRST TYPE
}

Atanas Iliev Iliev

Department of Algebra

Institute of Mathematics

Bulgarian Acad. of Sciences

P.O. Box 373

1090 Sofia

Bulgaria

February 1991 
GEOMETRY OF THE FANO THREEFOLD OF DEGREE 10 OF THE FIRST TYPE

Atanas IIiev IIiev

Table of Contents

$\S$ 1. Preliminaries.

$\S 2$. Sore elementary incidences, connected with the Schubert calculus on the Grassmannian of the lines in $\mathrm{F}^{4}$.

$\S 3$. The elements of $X$ as three-tangents to the ramification hypersurface $R \subset \mathrm{P}^{4}$.

\& 4. A description of the conics on $X=X_{10}$.

$\S 5$. The family $G^{3}$ of rational normal cubic curves on $\mathrm{X}$.

§6. The factorfamily $\ell_{0}^{3}$ as a determinantal variety.

$\S 7$. The families $\ell^{3}$ and $\ell_{0}^{3}$ as zero schemes.

$\S 8$. Tangent Bundle Theorem for the family of rational normal cubics $\varphi^{3}$.

§9. Tangent Bundle Theorem for the family of conics on the Fano threefold $\mathrm{X}=\mathrm{X}_{10}$.

References . 
$\S 1$. Preliminaries.

(1.1) DEFINIMION. The smooth irreducible projective variety $X$ of dimension three over the field of complex numbers is called a Fano threefold, if the anticanonical sheaf $-K_{X}$ (resp. the anticanonical divisor $-K_{X}$ ) is ample.

Let $X$ be a Fano threefold and let $K_{X}$ be trie canonical divisor of $X$. From the Riemann-Roch formula and from the Kodaira vanishing theorem it follows, that:

(a) $h^{i}\left(X, O_{X}\left(-m \cdot K_{X}\right)\right)=0$, for $i=1,2$ and for any $m-i n-$ teger; or, for $i>0$, m $\geqslant 0$; or, for $i<3, m_{2}<0$;

(b) $h^{0}\left(x, O_{X}\left(-m \cdot K_{X}\right)\right)=\left(m(m+1)(2 m+1) \cdot\left(K_{X}\right)^{3}\right) / 12+$ $+2 m+1$.

In particular, $h^{i}\left(x, o_{X}\right)=0$ for $i>0$, and $h^{0}\left(X,-K_{X}\right)=\operatorname{dim}\left|-K_{X}\right|+1=-\left(K_{X}\right)^{3} / 2+1$.

(1.2) DErINITION. The integer $g=g(X)=-\left(K_{X}\right)^{3} / 2+1$ is called a genus of the Fano threefold $X$.

The Fano variety $X$ is called a Fano variety of the main series, if the anticanonical divisor $-K_{X}$ is very ample. Therefore the linear system $\left|-K_{X}\right|$ gives an embedding of $X$ in $P^{g+1}$ as a subvariety of degree $2 . g-2,(g \geqslant 3)$. The intersections of $\mathrm{X}$ with codimension 2 subspaces $\mathrm{P}^{\mathrm{g}-1} \subset \mathrm{P}^{\mathrm{g}+1}$ are canonical curves of degree $2 . g-2$ and of arithmetical genus $p_{a}=g(X)$.

The Fano variety $X$ is called a (Fano) variety of the $1^{\text {-st }}$ kind, if $\operatorname{Pic}(X) \simeq \mathbb{Z}$, where Pic $(X)$ is the Picard group of $X$. The Fano varieties of the $1^{- \text {st }}$ kind (i.e., with Pic $\simeq \mathbb{Z}$ ) are classified by V.A.Iskovskikh ([I1], [I2 $],[I 4]$ ) after G.Fano $([F 1],[F 2])$ and I.Roth $([R])$. 
In the papers [MM1], [MN2] of S.Nori and S.Mukai is Given a list of the Fano threefolds of rank Pic $\geqslant 2$ i.e., the Fano 3-folds of the $2^{-r d}$ kind). From a birational point of view both kinds of Fano threefolds are interesting, but non-trivial questions, connected with the problem of non-rationality, arise from the Fano 3-folds with Pic $\simeq \mathbb{Z}$. Such non-trivial examples are the cubic hypersurface in $p^{4}$ $([\mathrm{CG}],[\mathrm{B} 1])$, the quartic double solid $([\mathrm{W}],[\mathrm{C}],[\mathrm{m} 1]$, [T2], [V]), the complete intersection of three quadrics in $\mathrm{P}^{5}([\mathrm{~B}],[\mathrm{TN}],[\mathrm{D}])$, the two-sheeted covering of the cone over the Veronese surface $([\mathrm{T}],,[\mathrm{H}])$, the quartic hypersurface in $\mathrm{P}^{4}([\mathrm{IM}],[\mathrm{I} 3],[\mathrm{I}])$, etc.

(1.3) The Fano threefolds of the main series and of the $1^{\text {-st }}$ kind have genus $g \leqslant 12$. The 3-folds of $g=7$ and of $g \geqslant 9$ are rational. The variety of $g=8$ is interpreted as an intersection of the Grassmannian of the lines in $\mathrm{P}^{5}$, embedied by $\mathrm{P}$ ücker in $\mathrm{P}^{14}$, with a codimension 5 subspace $\mathrm{P}^{9} \subset \mathrm{P}^{14}$; the last is birational to a smooth cubic hypersurface in $\mathrm{P}^{4}$. The variety $\mathrm{X}_{8} \subset \mathrm{F}^{6}$ of genus 5 is a complete intersection of three quadrics in $P^{6}$, etc. (see $\left[\mathrm{I}_{1}\right],[\mathrm{B} 2]$ ). In particular, the Fano varieties $\mathrm{x}_{10} \subset \mathrm{p}^{7}$ of genus 6 are the last non-trivial examples of Fano threefolds for which Pic $\simeq \mathbb{Z}$ and which have very ample anticanonical class. They are divided into two types (see $[G]$ ) :

$1^{\text {-st }}$ type. An intersection of the Grassmannian of the lines in $\mathrm{P}^{4}$, embedded by Plücker in $\mathrm{P}^{9}$, with a codimension 2 subspace $P^{7} \subset P^{9}$ and with a quadric; 
$2^{\text {-nd }}$ type. An intersection of a cone ( in $\mathrm{P}^{7}$ ) over the threefold $x_{5} \subset P^{6}$ with a quadric, where $x_{5} \subset P^{6}$ is an intersection of the Grassmannian of the lines in $P^{4}$, embedded by Plücker in $P^{9}$, with a codimension 3 subspace $F^{6} \subset P^{9}$.

It is known, that the two types of Fano threefolds of genus $g=6$ belong to one type of deformations ( $[G],[I 4])$.

The Fano threefold of genus $g=6$ (resp. of degree $d=2 . g-2=10$ ) of the first of the abovementioned types is studied in the present paper.

(1.4) The Fano threefold of degree 10 of the $1^{\text {-st }}$ type is represented as a 3-dimensional family of lines, lying in $\mathrm{P}^{4}$ (see for ex. $\S(1.5)$ and $\S 2$ ). In $\S 2$ are derived some elementary incidences, which are fulfiled for the lines $I \subset \mathrm{P}^{4}$, belonging to $\mathrm{X}=\mathrm{X}_{10}$ (see Propositions (2.2.2), $(2.2 .3),(2.2 .4))$. It is showm also the existence of a special hypersurface $R=R_{6} \subset P^{4}$ such that the lines $I \subset P^{4}$, which belong to $X$, are at least three-tangents to $R_{6}($ see $(2.2 .5)$ and $(2.2 .6))$. In $\S 3$ is considered a two-dimensional analogue of the threefold $\mathrm{X}_{10}$. In fact, this is the Del Pezzo surface $S=S_{2.2}$ in $P^{4}$, represented as an intersection of the Grassmannian $G(2,4)=$ $=G\left(1: P^{3}\right)$, embedded as a Plücker quadric in $P^{5}$, with a hyperplane $P^{4} \subset P^{5}$ and with a quadric (see Example 3.2.). In this case, the lines $I \subset P^{3}$, which belong to $S$, are at least bi-tangents to a Kummer surface in $P^{3}$ (see (3.2.7)). 
In the rest of $\S \cdot 3$ is shown, that the propositions from $\S 2$ can be extended in an appropriate way (see Cor. (3.3.3)). $\S 5,6,7$ are devoted to a detailed study of the family $\mathrm{C}^{3}$ of rational normal cubic curves, lying on the threefold $X=X_{10}$. First, in $\delta 5$ a geometrical investigation of the possible bundles of lines in $\mathrm{P}^{4}$, which represent the cubics $c \in l^{3}(X)$, is made (see $\S 5.2$.). It is shown also the existence of a well-defined involution $\sigma: \zeta^{3} \longrightarrow \zeta^{3}$, inducing a natural embedding of the factorfamily $C_{0}^{3}=e^{3} / \sigma$ in the Grassmannian $G=$ $=G(2,5)$ ( see Cor. $(5.3 .2)$ ). It is shown in $\S 6$, that the factorfamily $l_{0}^{3}$ can be embedded in a special projective bundle over the Grassmannian $G=G(2,5)$ as a degeneration locus (more precisely, as a second determinantal) of an appropriate net of quadrics over the Grassmannian ( see $\S 6.3$. , $\S 6.4$. and Prop. (6.4.4)). The essential results in $\S 7$ are the Corollaries (7.2.4) and (7.2.6), the Proposition (7.3.2) and their geometrical description in the Corollary (7.3.9). They are technical consequences from the results in $\S 6$, but under some additional open conditions on the variety $x_{10}(\operatorname{see}(6.3 .5)$ (i), (ii) and $(7.1 .2)$ (iii)). In particular, it is shown, that the family $\ell^{3}$ of rational normal. cubic curves on $\mathrm{x}_{10}$ can be embedded naturally in the projectivized bundle $P_{Z}\left(\Sigma^{*}\right)=G_{Z}(4, \Sigma)$ as a zero-scheme of some section ( see Prop. (7.3.2)) ; see 
also (6.3.2) and (6.3.3) for the definitions of $z$ and The geometrical description of the embedding $\varphi^{3} \hookrightarrow P_{2}\left(\Sigma^{*}\right)$, given in Corollary $(7.3 .3)$ is used in the formulation and in the proof of the Tangent Bunale Theorem (T.B.T.) for the family $\zeta^{3}$, stated and proven in $\S 8$ ( see Proposition (8.1.9) anà Theorem (8.2.12) ). The last describes the tangent bunale of the family $e^{3}$ by means of some standard bundies, defined over $\ell^{3}$ ( see (8.2.12) and Remark (8.2.10)). In fact, the T.B.T., in its formulation presented in the paper, describes the disposition of the tangent spaces at the points of the Abel-Jacobi image of the family $e^{3}$, inside the Intermeciate Jacobian $J(X)$ of $X$.

In addition, some known results on the family of conics on $\mathrm{x}_{10}$ are followed out in $\S 4$ and in $\S 9$. The original approach to the family of conics, which inspired the : author to go on further and to apply an appropriate technique to the family of rational normal cubics on $\mathrm{x}_{10}$, is due to D. Iogachev ( see $\left[\mathrm{I}_{1}\right],\left[\mathrm{J}_{2}\right]$ and also $(9.2 .1)$, $(9.2 .5),(9.3 .6),(9.3 .8),(9.3 .10),(9.3 .11))$.

The use and the possible applications of the T.B.T. to the study of the principally polarized Intermediate Jacobian of $X$ (see for ex. [CG]) are made in the author's comments in $\S 9.1$. 
(1.5) Let $V=V_{5}$ be the five-dimensional space $\mathbb{C}^{5}$ and let $G=G(2,5)=G(2, V)=G(1: P(V))$ be the Grassmannian of the two-dimensional subspaces of $V$ (resp. of lines in $F(\nabla)=$ $=\mathrm{P}^{4}$ ). There is a standard Flücker embedding:

PI: $G=G(2, V) \longleftrightarrow P^{9}=P\left(\Lambda^{2} V\right)$, which can be cescribed as follows:

Let $0 \subset I \subset V$ be a 2-dimensional subspace of $V$ (i.e. $I \in G$ ), and let $I=\mathbb{C} \cdot u+\mathbb{C} \cdot v$ for some basis $\{u, v\}$ of $I$. Then, by definition, PI: I $\longmapsto$ (the 1-dim. subspace $\left.0 \subset \mathbb{C} . u \wedge \nabla \subset \wedge^{2} \mathrm{~V}\right)$, i.e. $P I(I) \in G\left(1, \wedge^{2} V\right)=$ $=F\left(\Lambda^{2} V\right)=P^{9}$. It is easy to see that the definition of $F I(I)$ is independent of the choice of the basis $\{u, v\}$ of $I$. Let $D^{4}=P(V)$ has homogeneous coordinates $x_{0}, x_{1}, \ldots$ $\ldots, x_{4}$, and let $e_{0}, e_{1}, \ldots, e_{4}$ the corresponding dual basis of vectors in $V$. Let $e_{i j}=e_{i} \wedge e_{j}, 0 i \leqslant j \leqslant 4$ and let $\left\{\mathbf{x}_{i j}\right\}$ be the corresponding to $\left\{\mathbf{e}_{i j}\right\}$ dual basis. The embedded Grassmannian $G=G(2, V) \subset P\left(\wedge^{2} V\right)$ is described as the set of all classes of decomposable 2-forms (bi-vectors) in $\Lambda^{2} \nabla$ modulo a multiplication with an element of $\mathbb{C}^{*}$. Therefore, the ideal $I(G)$ of $G \subset P^{9}$ is denerated by the five Plucker quadrics $P I_{\text {m }}=x_{i j} \cdot x_{k I}-x_{i k} \cdot x_{j I}+x_{i I} \cdot x_{j k}$, $0 \leqslant \mathrm{~m} \leqslant 4, \quad 0 \leqslant i<j<\mathrm{k}<1 \leqslant 4$ and $i, j, k, 1 \neq m$. The Flücker embeding represents the Grassmannian $G$ as a smooth subvariety $G \subset P^{9}$ of degree 5 and of dimension 6 . Let $P^{7} \subset P^{9}$ be a codimension 2 subspace of $P^{9}=$ $=P\left(\Lambda^{2} \nabla\right)$, which is in general position with the embedded Grassmannian $G$, let $H_{1}$ and $H_{2}$ be two hyperplanes in $P^{9}$ such that $\mathrm{H}_{1} \cap \mathrm{H}_{2}=\mathrm{P}^{7}$ and let $Q$ be a general (esp. smooth) quadric in $P^{9}$ (or, equivalently, in $P^{7}$ ). According to the 
choice of the $P^{7}$ and. $Q$, the variety $X=X_{10}=G \cap P^{7} \cap Q=$ $=G \cap \mathrm{H}_{1} \cap \mathrm{H}_{2} \cap Q$ is smooth, and it is easy to see, that $X$ is a Fano variety of dimension 3 , of degree 10 and of genus 6 ; the anticanonical divisor $-K_{X}$ represents the hyperplane section of the embedded $X=X_{10} \subset P^{7}$.

\section{§ 2. Some elementary incidences, connected with the Schubert calcuius on the Grassmannian of the lines in $\mathrm{p}^{4}$.}

2.1. It is well known, that the integer homologies of the Grassmannian have no torsion and are generated freely by the homology classes of the Schubert cycles (see for ex. [GH] or [F]). In particular, the Schubert classes $\sigma_{a, b}$, $3 \geqslant a \geqslant b \geqslant 0$ ( of real codimension 2. $(a+b)$ ) of the Grassmannian $G=G(2,5)=G(2, \nabla)$ are described as follows:

Let $\mathrm{F}^{4}=\mathrm{P}(\mathrm{V})$ for $\mathrm{V}=\mathbb{C}^{5}$ and let $\mathrm{P}^{0} \subset \mathrm{P}^{1} \subset \mathrm{P}^{2} \subset$ $C P^{3} \subset P^{4}=P(\nabla)$ be a flag in $P(\nabla)$. Using the natural isomorphism: $G(2, V) \simeq G(1: P(V))$, which identify the two-dimensional subspace $O \subset I \subset V$ and the projective line $I=P(I) \subset$ $C P(V)$, we define $\sigma_{a, b}=\left\{I: I\right.$ is a line in $P^{4}$ and $\left.I \cap P^{3-a} \neq \varnothing, I \subset P^{4-b}\right\}$, where $3 \geqslant a \geqslant b \geqslant 0$.

The intersections of cycles in the Chow ring A. (G) can be described by the Pieri-formula (see for ex.[GH,ch.I,§5]):

$$
\sigma_{a, b} \cdot \sigma_{c, 0}={ }_{a+e=a+b+c, d \geqslant a \geqslant e \geqslant b} \sigma_{d, e} \cdot
$$

The intersection of general cycles $\sigma_{a, b} \cdot \sigma_{c, a}$ can be obtained from the Pieri-formula by the additive properties of the intersections of cycles (see for ex. the Giambelli formula in $[\mathrm{GH}, \mathrm{ch} . I, \S 5]$ ). 
2.2 .

$(2,2.1)$ Iet $\mathrm{V}=\mathrm{V}_{5}, \mathrm{P}^{4}=\mathrm{P}(\mathrm{V}), \mathrm{P}^{7} \subset \mathrm{P}^{9}=\mathrm{P}\left(\wedge^{2} \mathrm{~V}\right), \mathrm{H}_{1}$, $\mathrm{H}_{2}\left(\mathrm{H}_{1} \cap \mathrm{H}_{2}=\mathrm{P}^{7}\right), \mathrm{G}=\mathrm{G}(2, \mathrm{~V})=\mathrm{G}(1: \mathrm{P}(\mathrm{V})), \mathrm{Q}$ and $\mathrm{X}=\mathrm{X}_{10}=$ $=G \cap P^{7} \cap Q=G \cap \mathrm{H}_{1} \cap \mathrm{H}_{2} \cap Q$ be as above.

Let $W=W_{5}=G \cap P^{7}=G \cap H_{1} \cap H_{2}$. The fourfold $W$ is represented by the homology class $\sigma_{1,0} \cdot \sigma_{1,0}$ in the Chow ring A. (G), i.e. $W=\sigma_{1,0} \cdot \sigma_{1,0}=\sigma_{2,0}+\sigma_{1,1}$ (here = means the homology equivalence of cycies). In Iact, the homology cycle of $\sigma_{1,0}$ represents the hyperplane section according to the Plücker embedding, so the quadrics are represented by the cycle. 2. $\widetilde{\sigma}_{1,0}$

Let $x$ be a general point of $\mathcal{P}^{4}$. Using the fact, that the set of the lines in $P^{4}$, which pass through $x$, is represented by the cycle $\sigma_{3,0}(x)=\{I \in G: \mathbf{x} \in I\} \quad$ ( $\mathbf{x}$ is the $P^{0}$. in the flag, see above), and the homological equivalence $Q=2 . \sigma_{1,0}$, we derive the following:

$(2.2 .2)$ PROPOSITION.

(1) The set of the lines in $\mathrm{P}^{4}$, which belong to $W$ and which pass through a general point $x \in \mathbb{P}^{4}$ is homologous to the cycle $\sigma_{3,2}$, which represents a plane bundle of lines with a center the point $x \in \mathrm{P}^{4} \quad$ (i.e. $\sigma_{3,2}$ is a Grassmann line in $G$ ) ;

(2) There are exactly two lines in $\mathrm{P}^{4}$, which belong to $\mathrm{X}$ and which pass through the general point $x \in \mathrm{P}^{4}$.

Proof. It rests only to apply the formulae for the intersections of cycles on $G$.

Let now $\mathrm{P}^{3} \subset \mathrm{P}^{4}$ be a general hyperplane. Using the fact, that the set (thie Grassmannian) of all the lines in the 
choosen $P^{3}$ is represented by the cycle $\sigma_{1,1}\left(P^{3}\right)=$ $=\left\{I \in G: I \subset P^{3}\right\} \quad\left(P^{3}\right.$ is the $P^{3}$ in the flag, see above), we obtain in a similar way:

(2.2.3) PROPOSITION.

(1) The set of the lines, which belong to $W$ and which lie in a fixed generally choosen hyperplane $\mathrm{P}^{3} \subset \mathrm{P}^{4}$, is homologous to the cycle $\sigma_{3,1}+\sigma_{2,2}$ and represents a twodimensional quadric $Q\left(P^{3}\right)=S\left(P^{3}\right)$, embedied in $G$;

(2) The set of the lines, which belong to $X$ and which lie in a fixed generally choosen $P^{3} \subset P^{4}$ is homologous to the cycle 4. $\sigma_{3,2}$ and represents an elliptic curve $C\left(P^{3}\right)$ of degree 4 in $G$. The curve $C\left(P^{3}\right)$ is an intersection of the quadric $Q\left(P^{3}\right)$ with the quadric $Q$ in the 3-dimensional projective space $Q\left(P^{3}\right)=\operatorname{Span} Q\left(P^{3}\right)$.

From another point of view, the quadric surface $Q\left(P^{3}\right)$ is an intersection of the four-dimensional Plücker quadric $G\left(1: P^{3}\right)$, embedded in $G\left(1: P^{4}\right) \subset P^{9}$ by the natural embedding: corresponding to the embeding $P^{3} \subset P^{4}$, with the subspace $\mathrm{P}^{7} \cap \mathrm{P}^{9}$. From the last it follows immediately, that, if $\mathrm{P}^{3}$ is choosen sufficiently general, then the quadric $Q\left(P^{3}\right)$ and the curve $C\left(P^{3}\right)$ are smooth subvarieties of $G$. As a set of lines in $P^{3} \subset P^{4}$, the quadric $Q\left(P^{3}\right)$ is described as the set of all the lines in $P^{3}$, which intersect the both of a given pair of lines $I_{1}$ and $I_{2}$ in $P^{3}$, which does not intersect between them.

Iset now $I \subset \mathrm{P}^{4}$ be a generally choosen line, and let $\sigma_{2,0}(I)=\{\mathrm{m} \in \mathrm{G}: \mathrm{m} \cap I \neq \phi\}\left(I\right.$ is the $\mathrm{P}^{1}$ in the flag, as above). In a similar way, we can prove the analogues 
of the propositions above, namely:

(2.2.4) PROPOSITION. Let $I$ be a general line in $\mathrm{P}^{4}$. Then:

(1) The set of all the elements of $W$, which intersect as lines in $\mathrm{P}^{4}$ the line 1 , is homologous to the Grassmann cycle 2. $\sigma_{3,1}+\sigma_{2,2}$ and represents a linearly normal surface $S_{1}$ of degree 3 ; more precisely, the surfàce $S_{1}$ is embedded as a rational normal ruled surface $S_{1}=\mathbb{F}_{1}$ in $G$ by the Iinear system $\left|c_{0}+2 . f\right|$, where $C_{0}$ is the $(-1)$-section and $f$ is the fiber of $\mathbb{F}_{1}$;

(2) The set of the elements of $X$, which intersect as lines in $P^{4}$ the line $I$, is homologous to the cycle $6 . \sigma_{3,2}$ and represents a linearly normal curve $c_{1}$ of degree 6 and of (arithmetical) genus 2 .

(2.2.5) REMARKS.

(1) It is clear, that the curve $C_{1}$ is an intersection of the surface $S_{I}$ with the quadric $Q$ in the four-dimensional projective space $\mathbf{P}^{4}(I)=\left\langle S_{1}\right\rangle=$ span $S_{1}$. The hyperelliptic linear system $g_{2}^{1}$ on the curve $C_{1}$ is described in concrete geometrical terms as follows:

Let $x$ be a general point of 1 . There are exactly two lines $I^{\prime}(x)$ and $I^{\prime \prime}(x)$, which are elements of $X$ and which pass through $x$ (see Prop. $(2.2 .2)$ ), in particular $I^{\prime}(x)$ and $I^{\prime \prime}(x)$ belong to $C_{1}$. The linear system $g_{2}^{1}=$ $=\left|I^{\prime}(x)+I^{\prime \prime}(x)\right|$ maps $c_{1}$ twice on the projective line $I \subset P^{1}$; : the point $x \in I$ has as preimages the two lines $I^{\prime}(x)$ and $I^{\prime \prime}(x)$ as elements of $C_{I}$.

(2) As in the previous case, it is easy to see that the curve $C_{1}$ is non-singular under the general choice of the line $I \subset \mathrm{P}^{4}$. 
(3) Let $I$ be choosen so that the curve $C_{1}$ is smooth. Then the hyperelliptic projection $g_{2}^{1}$ for $C_{1}$ has exactly 6 ramification points: $\mathbf{x}_{1}, \mathbf{x}_{2}, \ldots, \mathbf{x}_{6}$; the last means, that the corresponding lines $I^{\prime}\left(x_{i}\right)$ and $I^{n}\left(x_{i}\right)$ (see (1)) coincide, $i=1,2, \ldots, 6$.

The arosed situation outlines the existence of a hypersurface $R=R_{6} \subset P^{4}$ of degree 6 , which is defined as the closed completion of the set of all the points ' $x_{i}$, $i=1,2, \ldots, 6$ of ramification of the hyperelizptic systems $g_{2}^{1}=g_{2}^{1}(I)$ (see (3)) corresponding to the Iines $I \in X$, for which $C_{I}$ is smooth.

(2.2.6) DEFINITION - COROIJARY. The closed completion $R=\overline{R^{0}}$ of the set $R^{0}=\left\{x \in P^{4}\right.$ : there is exactly one line ( a "double" line); which pass through $\mathbf{x}$ and which belongs to $X$ as an element of $G\}$ is a hypersurface of degree 6 in $P^{4}$. We call $R=R_{6}$ the ramification hypersurface for $X$.

$\S$ 3. The elements of $X$ as three-tangents to the ramification hypersurface $R \subset P^{4}$.

3.1. Let $I \subset P^{4}$ be a line, which belongs, as a point of the Grassmannian, to $X$. We shall show, that (when $I \in X$ is choosen sufficiently general) the line 1 has three points of a simple contact with the ramification hypersurface $R$ (see $(2.2 .6)$ ). The point here is not in proving this fact (by the way proving the threetangency is not so difficult), but in the suggestion that the geometrical interpretation of the elements of the subvariety $X$ of the Grassman- 
nian as lines, which are totally tangent to some hypersurface, is somewhat natural. The following example clarifies the situation in one more simple case.

3.2. Example.

Let $\mathrm{F}^{3}=\mathrm{P}\left(\mathbb{C}^{4}\right)$ be the projective 3 -space, and let $G(2,4)=G\left(2, \mathbb{C}^{4} ;=G\left(1: P^{3}\right)\right.$ be the Grassmannian of the Iines in $\mathrm{P}^{3}$, embedded as a Plücker quadric in the projective 5-space $p^{5}=P\left(\Lambda^{2} \mathbb{C}^{4}\right)$. Let $S=G\left(1: P^{3}\right) \cap H \cap Q$ be a smooth intersection of the embedded Grassmannian with the hyperplane $H=\mathrm{P}^{4} \subset \mathrm{F}^{5}$ and with the quadric $Q \subset \mathrm{P}^{5}$. Using, as in the case of $X$, the Schubert representation of $S$, we obtain easily:

(3.2.1) PROPOSITION.

(1) There are exactly two projective lines which pass through the general point $\mathbf{x} \in \mathrm{P}^{3}$ and which, as points of the Grassmannian $G\left(1: P^{3}\right)$, belong to $S$.

(2) There are exactly two lines,... wich lie in the generally choosen plane $\mathrm{P}^{2} \subset \mathrm{P}^{3}$ and which belong to $S$ as points of the $G\left(1: P^{3}\right)$.

(3) Let $I \subset \mathrm{P}^{3}$ be a.general Iine. Then the set $\mathrm{C}_{1}=$ $=\left\{m \subset P^{3}-a\right.$ line $: m \in S$ and $\left.m \cap I \neq \phi\right\}$, is an elliptic curve of degree 4 (the curve $C_{1}$ is, in the general case, a non-singular, though special element of the anticanonical system $\left|-K_{S}\right|$ )

(3.2.2) The four points of ramification $x_{1}, x_{2}, x_{3}$ and $x_{4}$ of the hyperelliptic Iinear system $g_{2}^{1}=\left|I^{\prime}(x)+I "(x)\right|$ (here $I^{\prime}(x)$ and $I^{\prime \prime}(x)$ are the two elements of $S$, which pass through the point $\mathbf{x} \in I$, see $\operatorname{Prop} .(3.2 .1)(1))$, 
define, just as in the case of $X$ (see Remark (2.2.5) (3)), a surface $R=R_{4} \subset P^{3}$ of degree 4 - the ramification (hyper) surface for $S$.

(3.2.3) Let now $P^{2} \subset \mathrm{P}^{3}$ be a sufficientiy generai piane. Let $I^{\prime}$ and $I^{\prime \prime}$ be the two Iines in $P^{2}$, which belong to $S$ as Grassmann points (see Frop. (3.2.1) (2)). It is easy to see, that there exists a rational map $\varphi: S \ldots . .>P^{2}$ such that:

(1) If $x \in P^{2} \backslash\{I \cdot \cup I "\}$, and: $x \notin R \cap P^{2}$, then $\varphi^{-1}(x)=\left\{I^{\prime}(x), I^{\prime \prime}(x)\right\}$, where $I^{\prime}(x)$ and $I^{\prime \prime}(x)$ are as above;

(2) If $x \in P^{2} \cap R$ and $x \notin I^{\prime} \cup I^{\prime \prime}$, then $\varphi^{-1}(x)=$ $=I(x)$, where $I^{(x)} I^{\prime}(x)=I^{\prime \prime}(x)$ is the unique element of $S$, which passes through $x$ as a line in $P^{3}$;

(3) If $x \in I^{\prime}$ (or if $\left.x \in I^{\prime \prime}\right)$, but $x \neq p=I^{\prime} \cap I^{\prime \prime}$, then $\varphi^{-1}(x)=\left\{I^{\prime}, I^{\prime \prime}(x)\right\} \quad$ (or resp. $\left.\varphi^{-1}(x)=\left\{I^{\prime \prime}, I^{\prime}(x)\right\}\right)$;

(4) If $x=p=I^{\prime} \cap I^{\prime \prime}$, then $\varphi^{-1}(x)=\left\{I^{\prime}, I^{\prime \prime}\right\}$.

(3.2.4) It is clear, that there exists a regularization $\pi$ of the rational map $\varphi$, which makes the following diagram commutative:

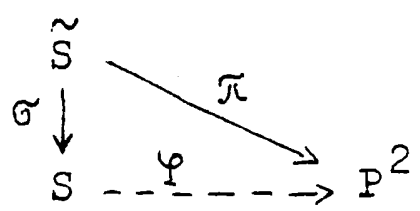

(3.2.5) In the diagram above the $\pi$-preimage of I' $U$ I" is an union of curves $I_{1}^{\prime}+I_{2}^{\prime}+I_{1}^{\prime \prime}+I_{2}^{\prime \prime}$ such that:

(i) If $\pi^{-1}(p)=\left\{p_{1}, p_{2}\right\}$, then $I_{1} \cap I_{1}^{\prime \prime}=p_{1}$, $I_{2}^{\prime} \cap I_{2}^{\prime \prime}=p_{2}, I_{1}^{\prime} \cap I_{2}^{\prime \prime}=\varnothing \quad, I_{1}^{\prime \prime} \cap I_{2}^{\prime}=\varnothing \quad ;$

(ii) The map $\sigma: \tilde{S} \longrightarrow S$ is a product of two $\sigma$-processes, which blow down the one of the two pairs of 
nor-intersecting lines (for ex. the lines $I_{1}^{\prime}$ and $I_{2}^{\prime \prime}$ ) to a pair of non-singular points ; moreover, the lines $I_{1}^{\prime}$ and $I_{2}^{n}$ are, of course, $(-1)$-curves on $S$.

It is clear now, that the lines $I^{\prime}$ and $I^{\prime \prime}$ are bi-tangents to the ramification curve $R \subset P^{2}$ of $\pi$.

(3.2.6) There are exactly 56 lines on the surface $\widetilde{S}$, which are $(-1)$-curves and which are also the 56 preimages of the 28 bi-tangent Iines to the plane quartic curve of ramification $R \subset P^{2}$; that is, the surface $S$ is a Del Pezzo surface, which is obtained from the projective plane after blowing-up of 7 points in the general position with respect to the lines, conics and cubics on the plane. On the other hand, there are exactly 16 lines $((-1)$-curves ) lying on the surface $S$.

Let $\mathrm{y}_{1}, \mathrm{y}_{2}, \ldots, \mathrm{y}_{7}$ be the seven points of the projective plane $P=P^{2}$, after blowing-up of which is obtained the surface $\tilde{S}$ and let the lines $I_{1}^{\prime}$ and $I_{2}^{\prime \prime}$ (see above) be the preimages of the points $\mathrm{y}_{6}$ and $\mathrm{y}_{7}$. Then the 16 lines on $S$ correspond to: (1) the conic, which pass through the points $\mathrm{y}_{1}, \mathrm{y}_{2}, \ldots, \mathrm{y}_{5} ;$ (2) the exceptional divisors over the points $\mathrm{y}_{1}, \mathrm{y}_{2}, \ldots, \mathrm{y}_{5} ;$ (3) the lines through the 10 pairs of points $\left(y_{i}, y_{j}\right), 1 \leqslant i<j \leqslant 5$.

(3.2.7) The interpretation of the surface $S$ as a subvariety of the Grassmannian $G\left(1: P^{3}\right)$ permits to get some interesting conclusions about the ramification surface $R \subset P^{3}$.

(1) Let $I_{0}$ be a line on the surface $S$. As a set of projective lines in $\mathrm{P}^{3}$, the grassmann line $I_{0}$ is described by a plane pencil of lines through a fixed point $x_{0}=$ $=x\left(I_{0}\right)$. The plane of this pencil $P_{0}^{2}=P^{2}\left(I_{0}\right)=$ 
$=\left(\bigcup \lambda: \lambda \subset P^{3}-a\right.$ line $\left.\& \lambda \in I_{0}\right)$ stands in a special position with respect to the ramification surface $R \subset F^{3}$. As tire line $I_{0} C S$ intersects five other lines $I_{1}, I_{2}, \ldots$ $\cdots, I_{5}$ Iying on the Del Fezzo suriace $S=S_{2.2}=$ $=S_{4} C P^{4}$, se there are five points $x_{1}, x_{2}, \ldots, x_{5}$ in the plane $F_{0}^{2}=P^{2}\left(I_{0}\right)$ each of them being the centre of the corresponding plane pencil of lines. In this case the analogue of tre map $\varphi$ from above $\varphi: S-\rightarrow \rightarrow F_{0}^{2}$ is a birational morphism. More concretely, by definition, the formal preimage $\varphi^{-1}(x)$ of the point $x \in P_{0}^{2}$ is defined to be the set of all the elements of $S$, which pass through $x$ as lines in $\mathrm{p}^{3}$. The regularization $\pi$ of the rational map $\varphi$ can be given by the commutative diagram

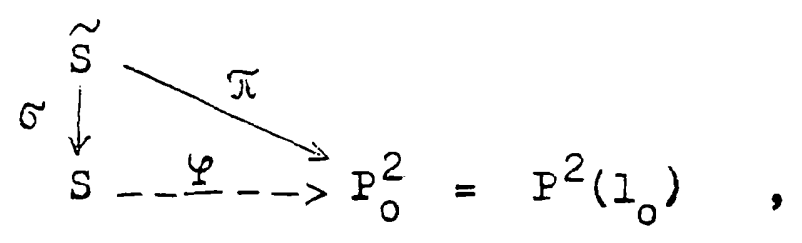

winere the regular map $\pi$ is a composition of six blow-ups over the points $x_{i}, i=0,1, \ldots, 5$, and the map $\sigma: \tilde{S} \longrightarrow S$ blows down the line $I \subset S$, which is the preimage of the conic $C$ in $P_{0}^{2}$, passing through the five points $x_{1}, x_{2}, \ldots, x_{5}$.

(2) The plane $P_{0}^{2}=P^{2}\left(I_{0}\right)$ is totally bi-tangent to the ramification surface $R$, along the conic $C$. It follows immediately from the description above that the projective lines, which are elements of $S \subset G\left(1: P^{3}\right)$ are bi-tangents to the ramification surface $R$; but not every bi-tangent to $R$ belongs to $S$. For examole, there are 28 bi-tangent 
lines to $R$, which lie in the general plane $\mathrm{P}^{2} \subset \mathrm{P}^{3}$, but only 2 of them belong to the surface $S \subset G\left(1: P^{3}\right)$. There are 16 planes, corresponding to the 16 lines on $S$, each plane being totally tangent to the suriace $R \subset P^{3}$; moreover, the 16 points, which are centers of the corresponding pencils of lines in $\mathrm{P}^{3}$, are singular points for the quartic $R \subset P^{3}$ and there are exactly 6 singular points of $\mathbf{R}$, which lie on a fixed totally tangent plane, etc.

(3.2.8) The facts, stated till now, give us a sufficient reason to claim, that the surface $R$ is a Kummer surface in $\mathrm{P}^{3}$; for example, there are 16 singular points on the quartic $R$, but the quartic surface with a maximal number of singular points is a surface of Kummer and the 16 singular points are simple nodes (see [B3, ch.VIII, ex.]).

The family of the bi-tangent lines to a Kummer surface splits into 28 (rational) components; 16 of them are isomorphic to the projective plane, and they are the dual planes to the 16 totally tangent planes to $R$; the rest 12 components are isomorphic to Del. Pezzo surfaces of degree 4 (as the surface $S$ ). The described set of 28 surfaces is a degeneration of the surface of the bi-tangents to a general quartic surface in $\mathrm{P}^{3}$, the last has been studied by Welters (see $[W$, part $1, \S 3]$ ) and Tikhomirov $\left(\sec \left[\mathrm{T}_{1}\right]\right)$. 
3.3. The elements of $x$ as three-tangent lines to $R$. (3.3.1) Let $I$ be a line in $\mathrm{P}^{4}$, which belongs, as a point of the Grassmannian $G=G\left(1: P^{4}\right)$, to $X$, and let $P^{2} \subset P^{4}$ be a sufficiently general plane through the line 1 . It follows from the adjunction formula, that the surface $S\left(P^{2}\right)=\left\{m \subset P^{4}\right.$ - a line $\left.: m \cap P^{2} \neq \varnothing\right\}$ is a K3-surface of degree 10 , which represents a (special) hyperplane section of the threefold $X=X_{10}$. It is easy to see, that we can choose the line $I$ and then the plane $P^{2}$ through 1 sufficiently general, such that the surface $S\left(P^{2}\right)$ be non-singular and moreover, such that the line $I$ be the unique element of $X$, which lies on the plane $P^{2}$. Iet $\varphi: S=S\left(P^{2}\right) \ldots \ldots P^{2}$ be, as in 8.2. , the natural rational map; that is, the formal preimage $\varphi^{-1}(x)$ of the point $x \in P^{2}$ is equal, by definition, to the set of all tine lines in $P^{4}$, which pass through $x$ and which belong, as elements of the Grassmannian, to $X$. In the abovedescribed case the rational map $\varphi$ can be regularized by the commutative àiagram

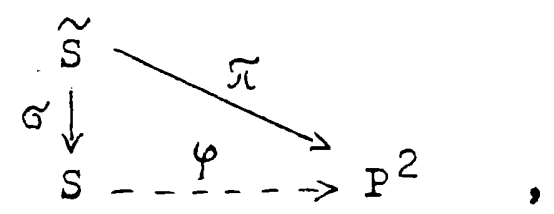

where $\sigma$ is a blowing-down of one of the two preimages of the line $I$, to a non-singular point of $S$ and where $\pi$ is a regular two sheeted covering with a ramification curve $\mathrm{R} \cap \mathrm{P}^{2}$ of degree 6 . It is clear, that the two $\pi$-preimages of the line $I$ are $(-1)$-curves on $\tilde{S}$ and hence, the line $I$ is a three-tangent to the ramification curve $R \cap \mathrm{P}^{2}$; in particular, the line $I$ is a threetangent line to $R$. 
(3.3.2) Let $\mathrm{P}^{5} \subset \mathrm{P}^{7}$ be any subspace of codimension 2 ; the intersection $C=X \cap P^{5}$ is a canonical curve of degree 10 and of arithmetical genus $p_{a}(c)=6$.

Let now $I \subset P^{3}$ be a pair of a line $I$ and a hyperplane $P^{3}$ through 1 . The curve $c=X \cap \sigma_{2,0}(I)+x \cap \sigma_{1,1}\left(P^{3}\right)=$ $=C_{I}+C\left(P^{3}\right)$ (see $\left.\S 2.2.\right)$ is an intersection of $X$ with a special codim. 2 subspace of $P^{7}$. In particular, the curve $C$ is a can. curve of $\operatorname{deg}(C)=10$ and of $p_{a}(C)=6$. The Schubert calculus gives, that $\operatorname{deg}\left(C_{1}\right)=6, \operatorname{deg}\left(C\left(P^{3}\right)\right)=$ $=4$ and $\left(C_{1}, C\left(P^{3}\right)\right)=4$. As $p_{a}\left(C_{m}\right)=2$ for the general line $m \subset P^{4}$ (see Prop. (2.2.4) (2)) and $P_{a}\left(C\left(P^{3}\right)\right)=$ $=1$ for the Eeneral hyperplane $\mathrm{P}^{3} \subset \mathrm{P}^{4}$ (see Prop. (2.2.3) (2)), then we derive from the numerical equalities above that $p_{a}\left(C_{1}\right)=2$ and that $p_{a}\left(C\left(P^{3}\right)\right)=1$ for any line $-1 \subset P^{4}$ and for any $\mathrm{P}^{3} \subset \mathrm{P}^{4}$. In fact, .. $\mathrm{p}_{a}\left(\dot{\mathrm{C}}_{1}\right) \geqslant 2$ and $p_{a}\left(C\left(P^{3}\right)\right) \geqslant 1$ follow from the correspondins equalities, mentioned just above. The equality $\left(C_{1}, C\left(P^{3}\right)\right)=4$ for $I C P^{3}$ implies that $p_{a}(C)=p_{a}\left(C_{1}\right)+p_{a}\left(C\left(P^{3}\right)\right)+3$. But $p_{a}(C)=6$, since $C$ is a canonical curve of aegree 10 . mherefore, the strong inequalities are impossible, that is $p_{3}\left(C_{1}\right)=2$ and $p_{a}\left(C\left(P^{3}\right)\right)=1$ for any line $I \subset P^{4}$ and for any hyperplane $\mathrm{P}^{3} \subset \mathrm{P}^{4}$.

(3.3.3) COROILARY. Let $I \subset P^{4}$ be any line, and let $P^{3} \subset P^{4}$ be any hyperplane. Let $C_{I}=\{m \in X$ - a line: $: \mathrm{m} \cap I \neq \phi\}, \mathrm{C}\left(\mathrm{P}^{3}\right)=\left\{\mathrm{m} \in \mathrm{X}\right.$ - a line: $\left.\mathrm{m} \subset \mathrm{P}^{3}\right\}$ ( see (2.2.3) (2) and (2.2.4) (2)). Then:

(1) $\mathrm{C}_{1}$ is a curve of degree 6 and of arithm. genus 2 ;

(2) $C\left(P^{3}\right)$ is a curve of degree 4 and of arithm. genus 1 . Noreover, if $I \subset P^{3}$, then the curves $C_{1}$ and $C\left(P^{3}\right)$ 
intersect between themselves in four points (with multiplicities) and the curve $C=C_{1}+C\left(P^{3}\right)$ is a canonical curve of aegree 10 .

(3.3.4) In particular, let the line $I$ be a three-tangent to the ramification hypersurface $R=R_{6} \subset P^{4}$. Then the normalization of the curve $C_{1}$ is $P^{1}$ and the line $I$ is a triple point of the curve $C_{I}$; moreover, the tangents to the three branches at the point $I \in C_{I}$ do not lie in one plane (see also the Corollary above).

\section{§ 4. A description of the conics on $\mathrm{X}=\mathrm{X}_{10}$.}

4.1. The family of the conics on the $F$ ano threefold $\mathrm{x}_{10}$ of the $1^{- \text {st }}$ type $(\operatorname{see}(1.3)$ ) is studied in the papers of $P$. Puts $[P]$ and $D$. Logachev $\left[I_{1}\right],\left[I_{2}\right]$. In many respects the analysis of the family $\ell^{3}$ of the rational normal cubics in the present paper is similar to Logachev's study of the family of conics on $\mathrm{x}_{10}$; because of that we shall give a brief expose of some results about the family of conics on $\mathrm{X}=\mathrm{x}_{10}$ "in the sense of Logacher" :

4.2. Let $X=G \cap H_{1} \cap H_{2} \cap Q=G \cap P^{7} \cap Q \subset P^{7} \subset P^{9}=$ $=P\left(\Lambda^{2} \nabla\right), \nabla=\nabla_{5}$ be as before. The hypersurfaces $H_{1}$ and $\mathrm{H}_{2}$, regarded as elements of $\mathrm{P}\left(\left(\Lambda^{2} \mathrm{~V}\right)^{*}\right)=\mathrm{P}\left(\Lambda^{2} \mathrm{~V}^{*}\right)$ (i.e., considered as one-dimensional subspaces of the vector space $\Lambda^{2} \mathrm{~V}^{*}$ ), determine a 2-dimensional subspace $\mathrm{E} C \cdot \Lambda^{2} \mathrm{~V}^{*}$. There exists a natural mapping $S: E \otimes V \rightarrow V^{*}$ defined as follows:

Let $\mathrm{H} \in \mathrm{I}$ and let $\mathrm{V} \in \mathrm{V}$; then $\mathrm{S}(\mathrm{H} \otimes \mathrm{v})=\mathrm{S}_{\mathrm{H}}(\mathrm{v}) \in \mathrm{V}^{*}$, where $S_{H}: \nabla \longrightarrow \nabla^{*}$ is the skew-symmetric linear mapping, corresponding to the element $H \in \Lambda^{2} \nabla^{*}$. The condition - 
- the variety $W=G \cap \mathrm{H}_{1} \cap \mathrm{H}_{2}$ to be smooth - means that tine equaity rank $S_{\mathrm{H}}=4$ is valid for any $\mathrm{H} \in \mathrm{E}$. Then tre 2-aimensioral subspace $E \subset \Lambda^{2} \mathrm{~V}^{*}$ defines the projective line $P(E)$ and the embeding $\psi_{0}: F(E) \longleftrightarrow P(V)$, where 0 is defined by the rule: $\psi_{O}(\mathrm{I})=\mathrm{Yer}_{\mathrm{H}}, H \in E$. In fact, Ker $S_{H}$ is one-dimensional subepace of $V$ for any $H \in E$, since $W$ is smooth (see above); hence, the derinition of $\psi_{0}$ is correct. The image $C_{0}=\Psi_{0}(P(E))$ is a conic in $P^{4}=P(V)$. The projective plane $\mathrm{P}_{0}^{2} \subset \mathrm{P}^{4}$, spanned on the conic $\mathrm{C}_{0}$ corresponds to some fixed 3-dirensional subspace $U \subset V$, such that $P_{0}^{2}=P(U)$. The "dual" plane $\left(P_{0}^{2}\right)^{*}=P\left(U^{*}\right)$ represents the set of the projective lines, lying in the plane $P_{0}^{2}$, that is, $\left(P_{0}^{2}\right)^{*}$ is a $\rho$-plane, lying in the fourfold $\mathrm{W} \subset \mathrm{P}^{7}=\mathrm{H}_{1} \cap \mathrm{H}_{2}$; moreover, the $\left(\mathrm{P}_{0}^{2}\right)^{*}$ is the unique $\rho$-plane in $W$ (see $[P]$ or $\left[I_{1}\right]$ ).

Let $I_{0}=P\left((V / U)^{*}\right) \subset P\left(V^{*}\right)$ be the "ortogonai" line to. the plane $P_{0}^{2}=P(U) \subset P(V)$, that is, $I_{0}=\left\{\nabla_{4} \subset \nabla\right.$ - a four-dimensional subspace : $\left.U \subset V_{4} \subset V\right\}$. There exists a ratural isomorphism $s: C_{0} \stackrel{\sim}{\longrightarrow} I_{0}$, defined by trie rule : $s(v)=S(E \otimes v) \in P\left(V^{*}\right)$, for $v \in I_{0}$.

The element $\nabla \in C_{0}$ defines the Schubert cycle $\sigma(v)=$ $=\sigma_{3,1}(v, s(v))=\left\{l \in G=G\left(1: P^{4}\right): v \in I \subset s(v)\right\}$. The schubert cycle $\sigma(v)$ represents the set of all the projective lines, passing through the point $v \in C_{0} \subset P(V)$ and lying in the three-dimensional embedded subspace $\mathrm{P}^{3}(\mathrm{v})=\mathrm{s}(\mathrm{v}) \subset \mathrm{P}^{4}=\mathrm{P}(\mathrm{V})$, that is, $\sigma(\mathrm{v})$ is a $\sigma-p l a n e$ in the Grassmannian $G$ (ibid.). 
(4.2.1) CIAIM (Logachev, $\left.\left[I_{1}\right]\right)$. The set $\left\{\sigma(v): v \in C_{0}\right\}$ describes all the $\sigma$-planes, lying in the fourfold $w=$ $=G \cap \mathrm{H}_{1} \cap \mathrm{H}_{2}$. Moreover, for any $\mathrm{v} \in \mathrm{C}_{0}$, the set $I(\mathrm{v})=$ $=\sigma(v) \cap\left(P_{0}^{2}\right)^{*}=\sigma(v) \cap\left(S_{p a n} C_{0}\right)^{*}$ is a line, which is tangent to the dual conic $C_{0}^{*} \subset\left(P_{0}^{2}\right)^{*}$. For any $\nabla_{4} \in I_{0}$ the surface $S\left(P\left(V_{4}\right)\right)=W \cap \sigma_{1,1}\left(P\left(V_{4}\right)\right)$ is a degenerated quadric, which splits into two planes: $S\left(P\left(\nabla_{4}\right)\right)=$ $=\left(P_{0}^{2}\right)^{*} \cup G\left(s^{-1}\left(\nabla_{4}\right)\right)$. The opposite is also true, namely: If $S\left(P\left(\nabla_{4}\right)\right.$ ) splits into two planes, then $\nabla_{4} \in I_{0}$ (for the definition of $S\left(P\left(V_{4}\right)\right)$ see (2.2.3)).

4.3. Let now $q \subset \mathbb{X}$ be a conic, lying on the Fano threefold $X=G \cap \mathrm{H}_{1} \cap \mathrm{H}_{2} \cap Q$. As a subvariety of the Grassmannian $G=G(1: P(V))$, the conic $q$ represents an one-dimensional bundle of projective lines in $P^{4}=P(V)$, which is of degree 2 with respect to the cycle $\sigma_{1,0}$ corresponding to the hyperplane section of the embedded by Plücker Grassmannian $G \subset P^{9}$. Consequently, the surface $S_{q}=$ $=\left(\bigcup_{I}, I-\right.$ a line in $\left.P^{4} \& I \in q\right) \subset P^{4}$ is one of the following:

(1) $S_{q} \subset \operatorname{Span} \cdot S_{q}=P_{q}^{3} \subset P^{4}$ is a non-singular quadric surface and $q$ corresponds to one of the two bundles of lines on the quadric $S_{q}$, that is, $q$ is a $\tau$-conic on $\mathrm{X}$ (see $[\mathrm{P}])$;

(2) $S_{q} \subset \operatorname{span} S_{q}=P_{q}^{3} \subset P^{4}$ is a quadratic cone and $q$ corresponds to the one-dimensional family of lines, lying on the cone $S_{q}$, that is, $q$ is a 5 -conic on $X$ (ibia.): (3) Span $S_{q}=P_{q}^{2} \subset F^{4}$ is a plane and $q$ corresponds to 
the set of all the tangent lines to a fixed conic in $\mathrm{F}_{q}^{2}$, that is, $q$ is a $\rho$-conic on $x$ (ibid.).

Since $X=W \cap Q$ is a general intersection of $W$ with a quadric $Q \subset P^{7}=\mathrm{H}_{1} \cap \mathrm{H}_{2}$, we can suppose that the above cases of possible conics, Iyiñ on $X$, are realized "in general position" that is, in particular, we can suppose, that the $\rho$-conics (resp., the $\sigma$-conics) on $X=W \cap Q$ are obtained as intersections of the $\rho$-planes (resp., of the $\sigma$-planes) on $W$ with the quadric $Q$. In the last context, the opposite is also true ( see $[P]$ or $\left[\mathrm{I}_{1}\right]$ ). In fact, the general conic on $X$ is of type $\tau$; the $\widetilde{S}$-conics on $X$ correspond to the points of the curve $C_{0}$ ( see Claim $(4.2 .1)$ ), and there is an unique $\rho$-conic on $X$, which corresponds to the unique $\rho$-plane $\left(P_{0}^{2}\right)^{*}$ on $W$.

4.4. Let now $q$ be a $\tau$-conic or a $\mathbb{G}$-conic on $X$ ( irrat is, $q \neq$ the unique $\rho$-conic $q_{0}=\left(P_{0}^{2}\right)^{*} \cap Q$ on $\mathrm{X})$; in particular, $q \subset G\left(1: \mathrm{P}_{\mathrm{q}}^{3}=\operatorname{Span} S_{\mathrm{q}}\right)$. As we know (see (2.2.3) and (3.3.31), the surface $S\left(P_{q}^{3}\right)=$ $=W \cap \sigma_{1,1}\left(P_{q}^{3}\right)$ is a quadric and the curve $C\left(P_{q}^{3}\right)=$ $=S\left(P{ }_{q}^{3}\right) \cap Q \cap \mathbb{X}$ is a space curve of degree 4 and of arithmetical genus 1 . As $q$ is a component of the curve $C\left(P_{q}^{3}\right)$, there exists an additional conic $\bar{q} \subset X$, such tinat $C\left(P_{q}^{3}\right)=q+\bar{q}$ and $(q, \bar{q})=2$. In particular, $P_{q}^{3}=P_{\bar{q}}^{3}=P^{3}(q, \bar{q})$. The conic $\bar{q}$ can be $\tau$-conic, $\sigma$-conic, or $p$-conic. In fact, if $\bar{q}=q_{0}$, then the symbol $P_{\frac{q}{q}}^{z}$ does not make sense. Then, under $P \frac{3}{q}$ we mean the 3 -space $P_{q}^{3}$. The incidence $\bar{q}=q_{0}$ is possible 
iff $q$ is a $\sigma$-conic (see (4.2.1)). From the Claim (4.2.1) we derive also that in the case when $q$ is a $\mathbb{s}$-conic, the quadric $S\left(P_{q}^{3}\right)$ splits into two plane : $S\left(P_{q}^{3}\right)=\left(P_{0}^{2}\right)^{*}+$ $+\sigma\left(x_{0}(q)\right)$ (here $x_{0}(q) \in C_{0}$ is the center of the cone $\left(\bigcup_{1}: I \subset p^{4}-\right.$ a line \& $\left.I \in q\right)$, i.e. $\bar{q}=q_{0}$ is the unique $\rho$-conic on $X$. In the case, when $q$ is $a$ $\tau$-conic, $\bar{q}$ is also a. $\tau$-conic and the surface $S\left(P_{q}^{3}\right)=$ $=S\left(\mathrm{P}_{\bar{q}}^{3}\right)$ is a non-singular quadric surface in $W$.

(4.4.1) DEFINITION. The set of conics :

$$
F_{c}=F_{c}(X)=\{q-a \operatorname{conic}: q \subset X\}
$$

is called a surface of the geometrical conics on $Z$.

The aajective "geometrical" is necessive in the context of the following investigations; see, for example, the difficulties, arising from the attempts to define correctly the natural involution on the surface of the conics on $\mathbb{Z}$.

(4.4.2) DEFINITION. We call the set of pairs :

$F=F(X)=\left\{\left(q, \nabla_{4}\right): q\right.$ is a conjic on $Z$, and $v_{4}$ is a subspace of dim. 4 of $\nabla$, such that $\left.S_{q} \subset P\left(\nabla_{4}\right)\right\}$ an extended surface of conics on $X$ ( or, simply, a surface of conics for $Z$ ).

4.5. The mapping $q \longmapsto \bar{q}, q \neq q_{0}$ defines a birational isomorphism of $F_{c}$, which can be completed to an involution $i$ on the surface $F=F(X)$ in view of the following considerations :

(4.5.1) It follows immediately from the definitions of $F$ and $F_{C}$ that the projection $\left(q_{i}, v_{4}\right) \longrightarrow q$ on the first factor defines naturally a morphism $r_{F}: F \longrightarrow F_{C}$, winich is one-to-one over $F_{C} \backslash\left\{q_{0}\right\}$. The curve $r_{F}^{-1}\left(q_{0}\right) \subset F$ 
is described by the set of all the four-dimensional subspaces $\mathrm{V}_{4} \subset \mathrm{V}$, for which $\mathrm{P}\left(\mathrm{V}_{4}\right)$ contains the projective plane $P_{q_{0}}^{2}=P_{0}^{2}$. Evidently, tine curve $r_{F}^{-1}\left(q_{0}\right)$ is naturaliy isomorpinic to the curve of $\checkmark$-conics $\left(=\{q: q\right.$ is a $\sigma$-conic on $\left.X\} \subset F_{c}\right)$; moreover, the morphism $r_{F}$ is a $\sigma$-process over the point $q_{0} \in F_{c}$. In this way the mapping $\bar{\bullet}: F_{c} \longrightarrow F_{c}$ defines correctly the involution $i: F(X) \longrightarrow F(X)$.

(4.5.2) DEFINITION. We define $F_{0}=F_{0}(X)$ to be the factorsurface $F / i$ of $F$ under the involution $i$; that is $F_{0}$ is identified with the set of all (non-ordered) pairs of involutive elements of $F$.

4.6. The projection on the second factor $\left(q, V_{4}\right) \longmapsto V_{4}$ defines a morphism $\varphi: F \longrightarrow\left(P^{4}\right)^{*}:=P\left(V^{*}\right)$.

Let $P_{F}: F \longrightarrow F_{0}$ be the natural two-sheeted covering, defined by the involution $i$. Clearly, there exists a commutative diagram:

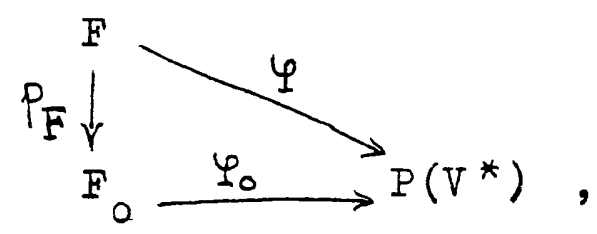

because the involutive elements of $F$ have same $\varphi$-images. It is easy to see also that the mapping $\varphi_{0}$ provides an embeding of the factorsurface $F_{0}$ in the projective space $P\left(V^{*}\right)$. 
$\S 5$. The family $\psi^{3}$ of rational normal normal cubic curves on $Z$.

5.1. PROPOSITION ( see $[G, \S 2$. Prop. 2.1 and 2.2]). $\left(\mathrm{X}=\mathrm{X}_{10}\right.$ is, as usual, a general Fano threefold of àegree 10 and of the $1^{\text {-st }}$ type, see $(1.3)$ )

(1) Let $C$ be a rational normal cubic curve on $\mathbf{X}$. Then the normal sheaf $\mathrm{N}_{C / X}$ is one of the following :

$$
N_{C / X}=\left\{\begin{array}{l}
o_{C} \oplus o_{C}(1) \\
o_{C}(-1) \oplus o_{C}(2) \\
o_{C}(-2) \oplus o_{C}(3) ; ;
\end{array}\right.
$$

(2) There are rational normal cubic curves on $\mathrm{X}$;

(3) Let $T$ be an irreducible reduced component of the family $\varphi^{3}$ of all the rational normal cubic curves on $x$, and let $\mathbf{x}$ be a sufficiently general point of $\mathrm{X}$. Then :

(a) $\operatorname{dim}\{C \in \mathbb{T}: C$ passes through $x\}=1$,

(b) The normal sheaf $\mathbb{N}_{C / X}$ of the general $C \in T$ is isomorphic to $O_{C} \oplus O_{C}(1)$.

5.2. A geometrical representation of the rational normal cubics on $Y$ as cubic scrolls.

(5.2.1) Let $C \subset X$ be a rational normal cubic, lying on the Fano threefold $X=G \cap H_{1} \cap H_{2} \cap Q$. The points of the curve $C$, regarded as lines in $P^{4}=P(V)$, sweep out a surface $S=S_{C}$ of degree 3 in $P^{4}$. The ruled surface $S_{C} \subset P^{4}$ is one of the following :

Case 1. $S_{C} \subset$ Span $S_{C}=P^{4}$, and :

(1.a) $S=F_{1}$ is a rational normal cubic scroll embedded in $\mathrm{P}^{4}$ by means of the linear system $|s+2 . f|$, where $s$ 
is the $(-1)-$ section and $f$ is the fiber of the ruled surface $\mathbb{F}_{1}=P\left(0_{P^{1}} \oplus O_{P^{1}}(1)\right.$,

(1.b) $S_{C}$ is a cone over a rational normal cubic curve $Z$ of degree 3 , with a center outside of the "ambient" space $\operatorname{Span} Z$;

Case 2. $S_{C} \subset \operatorname{span} S_{C}=P^{3} \subset P^{4}$, and :

(2.a) $S_{C}$ is a projection of a cubic scroll $\mathbb{F}_{1}$ (see (1.a) ) on $\mathrm{P}^{3} \subset \mathrm{P}^{4}$,

(2.b) $S_{C}$ is a projection of a rational cubic cone ( see (1.b) ) on $P^{3}$.

(5.2.2) CIAIM. The cases (1.b) and (2.b) do not occur for the general $X$.

Proof. We shall consider simultaneously the cases (1.b) and $(2 . b)$.

Let $S=S_{C}$ be a rational cubic cone ( in $P^{4}$, or resp. in some $P^{3} \subset P^{4}$ ), corresponding to some rational normal cubic $C \subset \mathbb{X}$. As we know ( see 4.2 ) the set of all $\sigma$-conics on $\mathbb{X}$ describes a rational curve on the surface of geometrical conics $F_{c}($ see Def. (4.4.1)). The lines, which are points of such a conic, sweep out a quadratic cone in $\mathrm{P}^{4}$. The rationaz curre on $F_{C}:$ which points corresponds to the $\sigma$-conics on $X$, is naturally isomorphic to the conic $C_{0}=\Psi_{0}(P(E))$ (see Claim (4.2.1)). Therefore, the set of the centers of the corresponding quadratic cones describes some rational curve $\hat{C}_{0}$ in $P^{4}$. In fact, the curve $\hat{c}_{0}$ is a non-singular conic, lying on the plane 
$P_{0}^{2} \quad($ see $\S 4.2$ and $\S 4.3)$; it is not hard to see also that the curve $\hat{c}_{0}$ coincides with the conic $C_{0}$ (see claim (4.2.1) ), but we don't need such a precision.

Let $x_{0}$ be the center of the cubic cone, corresponding to the cubic $C \subset X$ and let $y$ be a center of some quadratic cone, corresponding to some $\sigma$-conic $q$ on $x$. Let $I=\left\langle x_{0}, y\right\rangle$ be the line through the poirits $x_{0}$ and $y$. As we know, the curve $C_{1}=\sigma_{2,0}$ (I) $\Omega \mathbf{z}$ has a degree 6 and an arithmetical genus 2, independently of the choice of the line $l \subset P^{4}$ (see Corollary (3.3.3) (1); . Consequently, the curve $C_{1}$ has an additional component $I$ of degree 1 , such that $C_{I}=C+q+I$, where $C$ and $q$ are the cubic and the conic from above (all the lines of $C$ pass through $x_{0} \in I$, and all the lines of $q$ pass through $\mathrm{y} \in I$ ). As the curve $C_{I}$ is connected and all the projective lines, corresponding to the points of the "Grassmann" line $I$, intersect the line $I \subset P^{4}$, the center $z$ of the plane pencil of lines, which describes $I$, lies on the line $I=\left\langle x_{0}, y\right\rangle$. Obviously, the point $z$ is the unique point on the line $\left\langle x_{0}, y\right\rangle$ with the property, that $z$ is a center of some plane pencil of lines in $\mathrm{P}^{4}$, corresponding to some line, lying on $\mathrm{x}$.

The last considerations show, that the existence of a cubic $C \subset X$ of the type (1.b) or (2.b) implies an existing of an isomorphism between the rational curve $C_{0}$ ana some component of the curve $\Gamma$ (see $[P]$ or $[M]$ ), parametrizing the lines, lying on the Fano threefold $X$. But, as it is known $([P, \S 8])$, if $X$ is general, then 
the family $\Gamma$ has only one component ; moreover, the geometrical genus of $\Gamma$ is 71 , which leads us into a contradiction. Therefore, the cases (1.b) and (2.b) do not occur, if $X$ is sufficiently general .

(5.2.3) COROILARY. Let $C$ be a rational nomal cubic curve, lying on $X$. Then, the corresponaing to $C$ bunale of lines in $\mathrm{P}^{4}$ describes either the ruled surface $\mathbb{F}_{1}$, realizea as a cubic scroll in $P^{4}$, or some projection of a cubic scroll $\mathbb{F}_{1}$ on some $P^{3} \subset P^{4}$.

(5.2.4) In both the cases (1.a) and (2.a) there exists an unique line $I=I(C)$ in $P^{4}$, which coincides geometrically with the $(-1)$-section of the corresponding $\mathbb{F}_{1}$ (or of its projection) and such that the cubic curve $C$ is a component of the curve $C_{1}=\ldots \sigma_{2,0}$ (1) $\cap x$.

(5.2.5) On the other hand, $\operatorname{deg} c_{1}=6$ and $p_{a}\left(c_{1}\right)=2$. The last implies that there exists an additional rational normal cubic $\bar{C}$ on $\cdot \bar{x}$, such that $C_{I}=C+\bar{c}$ and $(C, \bar{C})=3$. It is possible curve $\bar{c}$ to be singular, which in our particular case means "a degenerated rational normal cubic" ; because of this, as in the case of the family of conics, we introduce the following refinement of the definition of the family of rational normal cubics on $\mathbf{X}$ :

$(5.2 .6)$ DEFINITION, We call the set $C^{3}=C_{e}^{3}(\mathrm{x})=\{c \subset \mathrm{x}$ : $\mathrm{c}$ is a rational normal (possibly, degenerate) cubic curve $\}$ a family of the rational normal cubic curves (or, simply r.n. cubics) on $\mathrm{X}$. 
5.3. The rational mapping $\mathrm{C} \longmapsto \overline{\mathrm{C}}$ defines an involution on the family of rational normal cubics on $X$. To be precise, we need to consider separately the special cases of "degenerate" rat. normal cubics on $\vec{x}$.

Let $c \in C_{e}^{3}$ be a degenerate r.n. cubic on $X$. We can separate the following possible cases for $C$ :

(i) $C=q+I$, where $q \in F_{C}(X)$ is a conic on $X$ and $I \subset X$ is a line, $\operatorname{such}$ that $(q, I)=1$;

(ii) $C=I_{1}+I_{2}+I_{3}$, where $I_{1}, I_{2}$ and $I_{3}$ are lines on $X$ and $\left(I_{1}, I_{2}\right)=\left(I_{2}, I_{3}\right)=1,\left(I_{1}, I_{3}\right)=0$. We shall find the involutive curve $\bar{C}$ in the cases and (ii).

Case (i') Let $q$ be a $\tau$-conic. Then the surface $\mathrm{s}_{\mathrm{q}}=\left(\bigcup_{1}, I \subset \mathrm{p}^{4}-\mathrm{a}\right.$ line $\left.\& I \in \mathrm{q}\right)$ is a nonsingular quadric and the set of lines $\{I: I \in q\}$ describes one of the two pencils of lines on $S_{q}$. Let $P_{I}^{2}$ be the plane $\left(\bigcup I, I \subset P^{4}\right.$ - a line $\left.\& I \in I\right)$, and let $y \in P_{I}^{2}$ be the center of the plane pencil of lines $\{I: I \in I\}$. The equality $(q, I)=1$ means that there exists a line $I_{0} \subset F^{4}$, which belongs to both of the pencils; evidently, $y \in I_{0}$. Let $I^{\prime}<S_{q}$ be the line from the second pencil of lines on $S_{q}$, which passes through the point $y$. Then the conic $q$ and the line $I$ are components of the curve $C_{I^{\prime}}=\sigma_{2,0}\left(I^{\prime}\right) \cap x$. Since $\operatorname{deg} C_{I^{\prime}}=6$ and $p_{a}\left(C_{I^{\prime}}\right)=2$, then the residue component $\bar{C}=C_{1},-q-I$ belongs to the family $\rho^{3}(x)$; moreover $(q+I, \bar{c})=3$, i.e. $\bar{c}=\overline{q+I}$ is the involutive of $C=q+I$. 
Case ( $i^{\prime \prime}$ ) Let $q$ be a $G$-conic. Then the surface $s_{q}=\left(\bigcup I, I \subset P^{4}-a\right.$ line \& $\left.I \in q\right)$ is a quadratic cone and the equality $(q, I)=1$ me ans that there is a line $I_{0}$, which is common for the pencils $\{I: I \in q\}$ and $\{I: I \in I\}$. Let $x_{0}$ be the singular point (the center) of the cone $S_{q}$ ana let $y$ be the center of the plane pencil of lines, corresponding to $I\left(\operatorname{see}\left(i^{\prime}\right)\right)$. Since $(q, I)=1$, then the plane of the second pencil $P_{I}^{2}$ does not lie in the subspace Span $\mathrm{S}_{\mathrm{q}} \subset \mathrm{P}^{4}$. In the opposite case the plane $P_{I}^{2}$ intersects the cone $S_{q}$ along a pair of lines $I_{0}$ and $I_{0}^{\prime}$. Both $I_{0}$ and $I_{0}^{\prime}$ are common members of the pencils $\{I: I \in q\}$ and $\{I: I \in I\}$, i.e. $(q, I)=2$ - a contradiction. Moreover, if the points $y$ and $x_{0}$ coincide, then the configuration $S_{q}+P_{I}^{2}$ corresponds to a degeneration of the case (1.b) (see (5.2.1)), which does not occur (see Claim (5.2.2)); the proof of the "degenerate" variant of the Claim (5.2.2) is, obviously, the same. Consequentiy, the points $x_{0}$ and $y$ determine the Iine $I_{0}=\left\langle x_{0}, y\right\rangle$. As in the case (i'), $C_{1}=q+I+\bar{C}$, where $(q+I, \bar{C})=3$, and the involutive curve $\bar{c}=\overline{q+I}$ belongs to $\ell^{3}$.

Case ( $i^{\prime \prime \prime}$ ) Let $q=q_{0}$ be the unique $\rho$-conic on $X$. Then, the center $\mathrm{J}$ of the burale $\{I: I \in I\}$ Iies on the plane $\mathrm{P}_{0}^{2}=\operatorname{Span} \mathrm{C}_{0}(\operatorname{see} \S 4.2)$. The points $\mathbf{v} \in \mathbf{C}_{\mathbf{a}}$ are centers of the bundles of lines in $\mathrm{P}^{4}$ corresponding to the Schubert cycles $(\mathbb{E}$-planes $) \mathbb{G}(v)=$ $=\mathbb{s}_{3,1}(\mathrm{v}, \mathrm{s}(\mathrm{v}))($ see $\S .4 .2)$ on the fourfold $W=$ $=G \cap \mathrm{H}_{1} \cap \mathrm{H}_{2} \subset \mathrm{H}_{1} \cap \mathrm{H}_{2}=\mathrm{P}^{7}$. The "Grassmann" 
quaaric $Q$ intersects each $\mathbb{S}$-piane $\mathcal{G}(v)$ in the $\widetilde{s}$-conic $q(v)=\widetilde{S}(v) \cap Q$; the center of the corresponding cone $S_{q(v)}$ coincides with the point $\nabla$, i.e. $x_{0}(v)=v, v \in C_{0}$. Iet now $I_{0} \subset I_{0}^{2}$ be a line through the center $y$ of the bundle $\{l: I \in I\}$. The line $I_{0}$ intersects the conic $c_{0}$ in two points: $v_{1}$ and $\mathbf{v}_{2}$; the last are the centers of the cones, correspond-

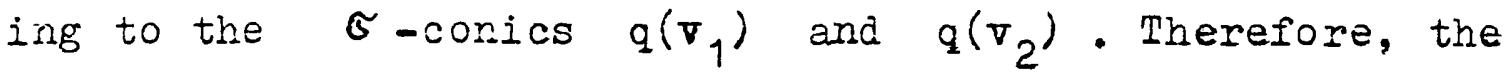
curve $C_{I_{0}}$ has as components the conics $q\left(v_{1}\right), q\left(\nabla_{2}\right), q_{0}$ and the line $I$ (the centers $\nabla_{1}, v_{2}$ and $y$ lie on $I_{0}$ and $I_{0}$. lies in the plane $P_{0}^{2}$ of the dual conic $\left.q_{0}\right)$. hence, $\sigma=\operatorname{deg} C_{I_{0}} \geqslant \operatorname{deg} q\left(\nabla_{1}\right)+\operatorname{deg} q\left(v_{2}\right)+\operatorname{deg} q_{0}+$ $+\operatorname{deg} I=7$ - a contradiction. Therefore, the case ( $\left.i^{\prime \prime \prime}\right)$ does not occur.

(5.3.1) RAMARK. As $q_{0}=\left(P_{0}^{2}\right)^{*} \cap Q$, the "Grassmann" conic $q_{0}$ is represented by the set of all lines in $P_{0}^{2}$ which are lines of intersection of the cones $S_{q(v)}, \nabla \in C_{0}$ and the plane $P_{0}^{2}$. For every $v \in C_{0}$ the cone $S_{q(v)}$ intersects $P_{0}^{2}$ in a pair of lines tinrough the point $v$; the set of all these lines coincides with the set of the tangent lines to some fixed conic $C_{0}^{1} \subset P_{0}^{2}$. In particular, every $\sigma$-conic $q(v), v \in C_{0}$ intersects its "involutive" $\overline{q(v)}=q_{0}$ in a pair of points (or, in a double point, if $\nabla$ is a point of an intersection of $c_{0}$ and $c_{0}^{\prime}$ ), which correspond to the pair of tangents to the conic $C_{0}^{\prime}$ through the point $v \in \mathrm{C}_{0}$.

Case (ii) Let the degenerate rational normal cubic $C$ splits into a chain of tinree lines : $C=I_{1}+I_{2}+I_{3}$, 
$\left(I_{1}, I_{2}\right)=\left(I_{2}, I_{3}\right)=1,\left(I_{1}, I_{3}\right)=0$. Let $P_{1}^{2}, P_{2}^{2}$ and $P \frac{2}{3}$ be the planes, and $y_{1}, y_{2}$ and $y_{3}$ be the centers of the corresponding to the "Grassmann" lines $I_{1}, I_{2}$ and $I_{3}$ plane pencils of lines in $\mathrm{P}^{4}$. The conditions for the intersections give, that the points $y_{1}$ and $y_{3}$ lie in the plane $\mathrm{P}_{2}^{2}$. The case $\mathrm{y}_{1}=\mathrm{y}_{2}=\mathrm{y}_{3}$ represents a special degeneration of the case (1.b) or (2.b) (see (5.2.1)), which does not occur for the general $x$ ( see Claim (5.2.2)). . If $\mathrm{y}_{1}=\mathrm{y}_{3} \neq \mathrm{y}_{2}$ or if $\mathrm{y}_{1}, \mathrm{y}_{2}$ and $\mathrm{y}_{3}$ are collinear, then the line $I=\left\langle y_{1}, y_{2}\right\rangle=\left\langle y_{3}, y_{2}\right\rangle$ is a common point of the "Grassmann" lines $I_{1}$ and $I_{3}$; but the last two do not intersect between them - a contradiction. Therefore, the line $I_{0}=\left\langle y_{1}, y_{3}\right\rangle$ is correctly defined and moreover $I_{0}$ does not pass through the point $\mathrm{y}_{2}$. Just as in the case (i), $C_{I_{0}}=I_{1}+I_{2}+I_{3}+\bar{c}$, where the curve $\bar{c}$ belongs to the family $\ell^{3}$, and

$$
\left(I_{1}+I_{2}+I_{3}, \bar{C}\right)=3 \text {, i.e. } \bar{C}=\overline{I_{1}+I_{2}+I_{3}} \text { is }
$$
the correctly defined involutive of $c=I_{1}+I_{2}+I_{3}$. (5.3.2) COROIIARY. Let $\ell^{3}=\zeta^{3}(\mathrm{x})$ be the family of the rational normal cubics on $X$. Then, there is a correctly defined involution $: \sigma: \zeta^{3} \longrightarrow \zeta^{3}$, such that $\sigma(c)=\bar{c}, c \in \zeta^{3}$. The factorscheme $\zeta_{0}^{3}=$ $=e^{3} / \sigma=\{$ the non-ordered pairs $(c, \bar{c})$ of involutive elements of $\left.\varphi^{3}\right\}$ is naturaily embedded in the Grassmannian $G=G(1: P(V))$ by the rule :

$$
(C, \bar{C}) \longmapsto 1 \text {, }
$$

where the line $I \subset P^{4}=P(V)$ is defined as above ( see 
cases (5.2.1) (1.a), (2.a) and $\S 5.3$ (i),(ii)); i.e. $I$ is the unique line in $P^{4}=P(V)$, such that $C_{1}=$ $=\widetilde{S}_{2,0}(1) \cap \mathrm{X}=\mathrm{C}+\overline{\mathrm{C}}$. The involutive pair of rationai normal cubics $C$ and $\bar{C}$ is defined equivalently by the numerical condition $(\mathrm{C}, \overline{\mathrm{C}})_{\mathbf{X}}=3$.

To prove the Corollary, it remains to note that the uniqueness of the line $I$ is demonstrated separately at every of the cases above; from the last it follows immediately that $\overline{\bar{c}}=c$ for every $c \in \mathrm{C}^{3}(\mathrm{X})$.

5.4. COMVIENTS.

(5.4.1) Let $(c, \bar{c})$ be an involutive pair of $\ell_{0}^{3}$ and let $c \cdot \bar{c}=t_{1}+t_{2}+t_{3}$, where $t_{1}, t_{2}$ and $t_{3}$ are points of $\mathrm{P}^{7}=\mathrm{H}_{1} \cap \mathrm{H}_{2}$, possibly with multiplicities. As $C$ and $\bar{C}$ are rational normal cubic curves, the intersection $C \cdot \bar{C}$ determines correctly an unique plane (the plane, "spanned on the intersection points of $C$ and $\bar{C} "$ ). Let $I \subset P^{4}$ be the line, such that $C_{1}=c+\bar{c}$, and let $p_{i}=t_{i} \cap I, i=1,2,3$ (the points $t_{1}, t_{2}$ and $t_{3}$ are regarded here as lines in $P^{4}$ ). It is evident that the line $I$ is a three-tangent to the ramification hypersurface $R=R_{6} \subset P^{4}$ (see Def.-Cor. (2.2.6)), that is $1 \cdot R=2 \cdot p_{1}+2 \cdot p_{2}+2 \cdot p_{3}$.

(5.4.2) The involutive pairs of the factorfamily $C_{0}^{3}$ as sections of the rational cubic scrolls $S_{I} \subset W$ with the quazric $Q$.

As above, let $W=G \cap \mathrm{H}_{1} \cap \mathrm{H}_{2}$ and let $I$ be a line in $P^{4}$. The intersection $S_{1}=\mathbb{S}_{2,0}(1)$. W is homolo- 
gous to the cycle 2. $\sigma_{3,1}+\sigma_{2,2}$ and represents a surface of aegree 3 ; moreover, for the general line $I \subset P^{4}$, the surface $S_{I}$ coincides with a surface $\mathbb{F}_{1}=P\left(0_{P^{1}} \oplus 0_{P^{1}}(1)\right)$, embedded in some four-dimensional subspace of $P^{7}=\mathrm{H}_{1} \cap \mathrm{H}_{2}$ as a rational normal cubic scroll (see Proposition (2.2.4) (1)). As $\mathbf{X}=$ $=W \cap Q$, where $Q$ is a (sufficiently general) quadric, the curve $c_{1}=\sigma_{2,0}(1) \cdot \bar{I}$ is an intersection of the surface $S_{I}$ with $Q$; in the notations of Prop. (2.2.4), the curve $C_{1}$ belongs to the linear system $\left|2 . C_{0}+4 . f\right|$ ( $C_{0}$ is the $(-1)$-section, $f$ is the fiber of $F_{1}$ ). The splitting $C_{I}=C+\bar{C}$ means that the restriction of the quadric $Q$ on the surface $S_{1}\left(=F_{1}\right.$, in the general case) can be represented as a sum of two hyperplane sections ( elements of tine linear system $\left|c_{0}+2 . f\right|$ ) of the cubic scroll $\mathbb{F}_{1}$. In the degenerate cases the surface $S_{1}$ is a degeneration of a family of $\mathbb{F}_{1}$ 's; we need to be careful with the symbols, because the surfaces $S_{C}$ in the cases (1.a) and (2.a) (see $\S 5.2)$ and also the surfaces $S_{q}+P_{I}^{2}$ and $P_{1}^{2}+P_{2}^{2}+P_{3}^{2}($ see $\S 5.3)$, which are cubic scrolls or their degenerations, represent cubic surfaces in $\mathrm{P}^{4}$, which are swept out by lines in $\mathrm{P}^{4}$ corresponding to the points of the curves $c \in \ell^{3}$. We shall study the conditions for the spliting $C_{1}=C+\bar{c}$ in detail in the next paragraph. 
\& 6. The factorfamily $\ell_{0}^{3}$

as a determinantal variety.

6.1. Tauthological sequences on $P\left(V^{*}\right)$ and on $G(2, V)$.

In the present section we need some details, concerning the well-known standaró tauthologicai sequences on the Grassmannians.

(6.1.1) We shall regard the "dual" projective space $\left(P^{4}\right)^{*}=P\left(V^{*}\right)$ as a Grassmannian $: P\left(V^{*}\right)=G(4, \nabla)=$ $=\left\{\nabla_{4}: \nabla_{4}\right.$ - a four-dimensional subspace of $\left.\nabla\right\}$. In this interpretation the standard tauthological sequence on $P\left(V^{*}\right)$ :

$$
0 \longrightarrow \tau_{4, \nabla} \longrightarrow F\left(\nabla^{*}\right) \times V \longrightarrow \tau_{1, \nabla^{*}}^{*} \longrightarrow 0
$$

parametrizes the family of embedings

$$
\left\{0 \rightarrow V_{4} \longrightarrow V: \nabla_{4} \in G(4, V)=P\left(V^{*}\right)\right\} .
$$

Let $f={ }^{t}\left(f_{1}, \ldots, f_{4}\right)$ and $g={ }^{t}\left(g_{1}, \ldots, g_{4}\right)$ be the vector-columns of two given bases of the subspace $\nabla_{4} \subset \nabla$ and let $f=A \cdot g$ be a change of the basis $f$ by the basis $g$. Then $\wedge^{4} f=f_{1} \wedge f_{2} \wedge f_{3} \wedge f_{4}=$ $=\operatorname{det} A \cdot g_{1} \wedge g_{2} \wedge g_{3} \wedge g_{4}=\operatorname{det} A \cdot \wedge^{4} \mathrm{~g} \cdot$ On the other hand, the Plucker embedding $P I\left(\Lambda^{4}\right): G(4, V) \longrightarrow P^{4}$ provides the natural isomorphism: $G(4, V) \simeq P\left(V^{*}\right)$; $P I\left(\wedge^{4}\right): V_{4} \longrightarrow \Lambda^{4} f$ (mod. proportionality by elements of $\left.\mathbb{C}^{*}\right), V_{4} \in G(4, V)$. From here we get immediately, that det $\tau_{4, V}=O_{G(4, V)}(-1)$, according to the embedaing above. Keeping in mind the last, we replace $G(4, V)$ with $\mathrm{P}\left(\mathrm{V}^{*}\right)$; in particular, we write $\operatorname{det}\left(\tau_{1, \mathrm{~V}^{*}}^{*}\right)=\tau_{1, \mathrm{~V}^{*}}^{*}=$ $=O_{G(4, V)}(1)=O_{P\left(\nabla^{*}\right)}(1)$. 
$(6.1 .2)$ I.et now $G=G(2, V)=\left\{V_{2}: V_{2}\right.$ is a z-dim. subspace of $V\}$ be the Grassmannian and let

$0 \longrightarrow \tau_{2, V} \longrightarrow G(2, \mathrm{~V}) \times \mathrm{V} \longrightarrow \tau_{3, V^{*}}^{*} \longrightarrow 0$

be the stancard tauthological sequerce on $G$. Over the element $V_{2} \subset V$ of $G(2, V)$ the emieajing of the left sicie coinciảes witin the naturai embeding $0 \rightarrow V_{2} \rightarrow V$. If we perform a base change $f=A \cdot g$ in the fiber $\nabla_{2}\left(f={ }^{t}\left(f_{1}, f_{2}\right)\right.$ and $g={ }^{t}\left(E_{1}, g_{2}\right)$ are two bases of $\mathrm{V}_{2}$, as above), we obtain a change of the second exterior powers $\Lambda^{2} f=\operatorname{det} A \cdot \Lambda^{2} g$.

On the other hand, the Plücker embedding

$$
P I\left(\Lambda^{2}\right): G(2, \nabla) \longrightarrow P\left(\Lambda^{2} \nabla\right)=P^{9}
$$

maps the element $\nabla_{2} \in G$ to the class $\Lambda^{2} f$ (mod. proporticnality by elements of $\mathbb{C}^{*}$ ). In view of the last, the former change means that $\operatorname{det}\left(\tau_{2, \mathrm{~V}}\right)=0_{G(2, V)}(-1)$, according to the Plucker embeding $\operatorname{PI}\left(\Lambda^{2}\right)$.

6.2. Pfaff ideals on $P\left(\nabla^{*}\right)$ and $G(2, V)$.

(6.2.1) We shall define a subbundle $P f$ of the bundle of quadrics $S^{2} \Lambda^{2} \tau_{4, \nabla}$ over $P\left(V^{*}\right)=G(4, V)$.

Let $\nabla_{4} \subset \nabla$ be an element of $P\left(V^{*}\right)=G(4, V)$, let $f={ }^{t}\left(f_{1}, \ldots, f_{4}\right)$ and $g={ }^{t}\left(g_{1}, \ldots, g_{4}\right)$ be, as above, bases of $\mathrm{v}_{4}$ and let $\mathrm{y}=\left(\mathrm{y}_{1}, \ldots, \mathrm{y}_{4}\right)$ and $z=\left(z_{1}, \ldots, z_{4}\right)$ be the corresponding bases of coordinates on $V_{4}$. Assigned to the base $f$, we define the fibre of $\mathrm{Pf}$ to be: $\mathrm{Pf}\left(\mathrm{V}_{4}\right)_{f}=$ the Plücker quadric $\mathrm{y}_{12} \cdot \mathrm{y}_{34}-\mathrm{y}_{13} \cdot \mathrm{y}_{24}+\mathrm{y}_{14} \cdot \mathrm{y}_{23}$, where $\mathrm{y}_{i j}$, $1 \leqslant i<j \leqslant 4$ are the coordinates, which correspond to the 
basis $f_{i j}=f_{i} \wedge f_{j}, 1 \leqslant i<j \leqslant 4$ of $\wedge^{2} \nabla_{4}$ ( the same for the coordinates $z_{i j}$ and for the base vectors $g_{i j}$ of $\left.\Lambda^{2} \nabla_{4}\right)$. Let $f=A \cdot g$ be a base change of $\nabla_{4}$; the change of the coordinates is $\mathrm{y} \cdot \mathrm{A}=\mathbf{z}$, i.e. $y=B \cdot z$, where $B=A^{-1}$. The last allows to compute the change of Pf : Pf $\left(\mathrm{V}_{4}\right)_{f}=\mathrm{y}_{12} \cdot \mathrm{y}_{34}-\mathrm{y}_{13} \cdot \mathrm{y}_{24}+$ $+\mathrm{y}_{14} \cdot \mathrm{y}_{23}=\cdots=\operatorname{det} \mathrm{B} \cdot\left(\mathrm{z}_{12} \cdot \mathrm{z}_{34}-\mathrm{z}_{13} \cdot \mathrm{z}_{24}+\mathrm{z}_{14} \cdot \mathrm{z}_{23}\right)=$ $=(\operatorname{det} A)^{-1} \cdot \operatorname{Pf}\left(V_{4}\right)_{g}$; but $A$ changes the bases of the bundle $\tau_{4, V}(\operatorname{see}(6.1 .1))$, hence $P f=\left(\text { cet } \tau_{4, V}\right)^{-1}=$ $=O_{P}\left(V^{*}\right)(1)$. Here we shall describe one geometrical interpretation of the bunde of quadrics $P f$.

Taking the second exterior power of the embedding $0 \longrightarrow \tau_{4, \nabla} \longrightarrow P\left(V^{*}\right) \times V$ from the tauthological sequence, we obtain the embedding

$$
0 \longrightarrow \Lambda^{2} \tau_{4, V} \longrightarrow P\left(V^{*}\right) \times \Lambda^{2} V,
$$

which parametrizes the family of embeddings $\Lambda^{2} \mathrm{v}_{4} \hookrightarrow \Lambda^{2} \nabla$, $\mathrm{v}_{4} \in \mathrm{P}\left(\mathrm{V}^{*}\right)$. The fiber $\mathrm{Pf}\left(\mathrm{V}_{4}\right)$ coincides with the one-dimensional vector space, spanned on the equation of the embedded Grassmannian $G\left(2, v_{4}\right)=\sigma_{1,1}\left(P\left(\nabla_{4}\right)\right) C$ $C \operatorname{span} \sigma_{1,1}\left(P\left(\nabla_{4}\right)\right)=P\left(\Lambda^{2} \nabla_{4}\right)$ as a subvariety of $G(2, V) \subset P\left(\Lambda^{2} V\right)=P^{9}$.

(6.2.2) Let we take the exterior product of the embedding $0 \rightarrow \tau_{2, V} \longrightarrow G(2, V) \times V$ with the constant bundle $G(2, V) \times V$. The obtained embedding

$$
0 \longrightarrow \tau_{2, V} \wedge \nabla \longrightarrow G(2, V) \times \wedge^{2} v
$$

can be interpreted geometrically as follows: 
Let $V_{2} \subset V$ be an element of $G(2, V)$ and let $\sigma_{2,0}\left(P\left(V_{2}\right)\right)$ be the schubert cycle $\{I \subset P(V)$ - a line: : I $\left.\cap \mathrm{P}\left(\mathrm{V}_{2}\right) \neq \phi\right\}=\{I \subset \mathrm{V}$ - a subspace of dim. $=2$ : $\left.: \operatorname{aim}\left(I \cap V_{2}\right) \geqslant 1\right\}$, embedded in $P\left(\Lambda^{2} \nabla\right)$ as a subvariety of the embedded Grassmannian $G(2, V)$. We can check directly that Span $\sigma_{2,0}\left(P\left(\nabla_{2}\right)\right)=P\left(V_{2} \wedge V\right)$. Now we can define the "ideal" subbundle $\mathbb{I} \subset \mathrm{s}^{2}\left(\nabla_{2} \wedge \nabla\right)$, namely:

$$
\mathbb{I}\left(\mathrm{V}_{2}\right)=\left[\text { the set of all the quadrics in } P\left(\mathrm{~V}_{2} \wedge \mathrm{V}\right)\right.
$$
(regarded as elements of $\mathrm{S}^{2}\left(\mathrm{~V}_{2} \wedge \mathrm{V}\right)^{*}$ ) which vanish on the subvariety $\sigma_{2,0}\left(P\left(V_{2}\right)\right)=$ $\left.=\mathrm{H}^{\circ}\left(\mathrm{P}\left(\mathrm{V}_{2} \wedge \mathrm{V}\right), 0\left(2-\sigma_{2,0}\left(\mathrm{P}\left(\mathrm{V}_{2}\right)\right)\right)\right)\right] c \mathrm{~S}^{2}\left(\mathrm{~V}_{2} \wedge \mathrm{V}\right)^{*}$ As in (6.2.1) we compute the cocycle of the base changes of the bundle $\mathbb{I}$; as a result we obtain that $\mathbb{I}$ is isomorphic to the bunaile $\tau_{3, V^{*}}^{*}$.

(6.2.3) COROLLARY.

(i) Let $\operatorname{Pf} \subset s^{2} \Lambda^{2} \tau_{4, V}^{*}$ be the sheaf of quadrics over $P\left(\nabla^{*}\right)$ with a fibre $P f\left(\nabla_{4}\right)=$

$$
=H^{\circ}\left(P\left(\Lambda^{2} V_{4}\right), O\left(2-\sigma_{1,1}\left(P\left(V_{4}\right)\right)\right)\right)
$$

over the element $V_{4} \subset V$ of $P\left(V^{*}\right)=G(4, V)$. Then there is a natural isomorphism :

$$
P f \simeq O_{P\left(V^{*}\right)}(1) \quad ;
$$

(ii) Iet II $\subset s^{2}\left(\tau_{2, v} \wedge v\right)$ be the bundle of quadrics over $G=G(2, V)$ with a fibre $\mathbb{I}\left(V_{2}\right)=$ $=\mathrm{H}^{\mathrm{O}}\left(\mathrm{P}\left(\mathrm{V}_{2} \wedge \mathrm{V}\right), \mathrm{O}\left(2-\sigma_{2,0}\left(\mathrm{P}\left(\mathrm{V}_{2}\right)\right)\right)\right)$ 
over the element $V_{2} \subset V$ of $G(2, V)$. Then there is a natural isomorphism :

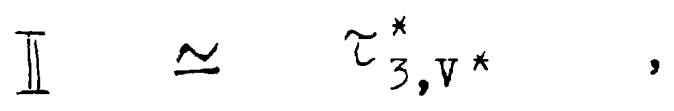

where $\tau_{3, V^{*}}^{*}$ is the tauthological factorbundle over $G(2, V)$.

(6. 1-2) COMMENTS. The bundies of quadrics $\mathrm{Pf} \longrightarrow P\left(V^{*}\right)$ and II $\longrightarrow G(2, V)$ are the components of degree 2 in the graded bundles of ideals of the families of the embedded Schubert cycles $\quad\left\{\sigma_{1,1}\left(P\left(V_{4}\right)\right) \subset P\left(\Lambda^{2} v_{4}\right): V_{4} \in P\left(V^{*}\right)\right\}$ and $\quad\left\{\sigma_{2,0}\left(F\left(V_{2}\right)\right) \in P\left(V_{2} \wedge V\right): V_{2} \in G(2, V)\right\}$ respectively. Obviously, the components $\mathrm{Pf}_{\mathrm{f}}$ and II generate the corresponding ideals.

6.3. The factorfamily $\zeta_{0}^{3}$ as a set of quadrics.

$$
\text { (6.3.1) Let } Q \subset S^{2}\left(\tau_{2, V} \wedge V\right)^{*} \text { be the }
$$

bundle of quadrics over $G=G(2, V)$, winich parametrizes tine family of restrictions of tine quadric $Q \in S^{2}\left(\Lambda^{2} V\right)^{*}$ on the subspaces of the form $\nabla_{2} \wedge \nabla, V_{2} \in G(2, V)$. As the quadric $Q$ is sufficiently general, the corresponding bundle of restrictions $Q\left(V_{2}\right)=\left[\right.$ the $\mathbb{C}^{*}$-class of the equation of the restriction of the quadric hypersurface

$$
\left.Q=0 \text { in } P\left(V_{2} \wedge V\right)\right] \text { is correctly defined. }
$$

(*) NOTE. We use the same symbol $Q$ for the ouadric $Q \in S^{2} \Lambda^{2} \nabla^{*}$, for the surface $(Q=0)$ in $P\left(\wedge^{2} \nabla\right)$ and for the bunde "of quadrics" $Q$ over $G(2, V)$.

obviously, the bundle $Q$, defined above, is trivial, i.e. the corresponding sheaf $Q$ is isomorphic to the structure

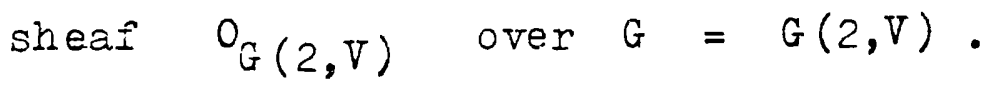


(6.3.2) Propositidun. Let $Z \subset P_{G}\left(S^{2}\left(\tau_{2, V} \wedge V\right)^{*}\right)$ be the set :

$$
z=P\left\{\left(V_{2}, q\right): V_{2} \in G, q \in H^{0}\left(P\left(V_{2} \wedge v\right), O\left(2-\sigma_{2,0}\left(P\left(V_{2}\right)\right), Z\right)\right)\right.
$$

Then $Z$ is naturally isomorpic to the projectivized burile $P_{G}\left(\tau_{3, V^{*}}^{*} \oplus O_{G}\right)$ over $G=G(2, V)$.

Procf. The proposition follows immediately from the Corollary (6.2.3) (ii) and from (6.3.1). It remains to see that the fiber of the natural projection $Z \longrightarrow G(2, \nabla)$ over the "point" $V_{2} \in G(2, V)$ coincides with the space $\operatorname{Span}\left\{\mathbb{I}\left(\mathrm{V}_{2}\right) \cup \mathrm{Q}\left(\mathrm{V}_{2}\right)\right\}=\mathbb{I}\left(\mathrm{V}_{2}\right) \oplus \mathrm{Q}\left(\mathrm{V}_{2}\right)$.

(6.3.3) Let now $\mathrm{X}=\mathrm{G}(2, \mathrm{~V}) \cap \mathrm{H}_{1} \cap \mathrm{H}_{2} \cap \mathrm{Q}$ and let $\nabla_{8} \subset \nabla_{10}=\Lambda^{2} \nabla$ be the subspace, such that $\mathrm{P}^{7}=$ $=\mathrm{H}_{1} \cap \mathrm{H}_{2}=\mathrm{P}\left(\mathrm{V}_{8}\right)$. We define a bundle $\sum$ over $G(2, V)$ with fibers $\sum\left(v_{2}\right)=\left(\nabla_{2} \wedge \nabla\right) \cap \nabla_{8}$ $\nabla_{2} \in G(2, \nabla)$. We can suppose that the subspace $P^{7}=P\left(\nabla_{8}\right)$ is choosen a "sufficiently general" in such a way, that all the intersections $\left(\nabla_{2} \wedge \nabla\right) \cap \nabla_{8}$ are transversal ( and, hence, are vector spaces of àimension 5 ) for every $v_{2} \in G(2, V)$. Considerations on the level "intersection of Schubert cycles"give that the last requirement is fulfilled for the elements $V_{8}$ of an open subset of the Grassmannian $G\left(8, V_{10}\right)$.

$$
\begin{aligned}
& (6.3 .4) \text { Let } V_{2} \in G(2, V) \text {. As we know, } S_{P}\left(V_{2}\right)= \\
= & \sigma_{2,0}\left(P\left(V_{2}\right)\right) \cap P\left(V_{8}\right)= \\
= & \left\{I \subset P^{4}-\text { a line }: I \in W, I \cap P\left(V_{2}\right) \neq \varnothing\right\} \text { (see }
\end{aligned}
$$

Proposition (2.2.4) (1) anà (5.4.2)). Let $\nabla_{8} \subset \nabla_{10}=$ 
$=\Lambda^{2} \mathrm{~V}$ be choosen general. Then, taking into account tine considerations above, we have that all the restriction maps :

$$
\begin{aligned}
\mathrm{H}^{\circ}\left(\mathrm{P}\left(\mathrm{V}_{2} \wedge \mathrm{V}\right), \mathrm{O}\left(2-\sigma_{2,0}\left(\mathrm{~F}\left(\mathrm{~V}_{2}\right)\right)\right)\right) \longrightarrow \\
\longrightarrow \mathrm{H}^{0}\left(\mathrm{P}\left(\sum\left(\mathrm{V}_{2}\right)\right), \mathrm{O}\left(2-\mathrm{S}_{\mathrm{P}\left(\mathrm{V}_{2}\right)}\right)\right),
\end{aligned}
$$

are isomorphisms. So we obtained

(6.3.5) COROLIARY. Let $\nabla_{8} \subset \nabla_{10}=\Lambda^{2} \nabla$ and $Q$ are choosen sufficiently general, such that :

(i) The variety $X=G \cap Q \cap P\left(\nabla_{8}\right)$ is smooth;

(ii) $\operatorname{dim}\left(v_{8} \cap\left(v_{2} \wedge v\right)\right)=5$, for every

$$
\nabla_{2} \in G=G(2, V) \text {. }
$$

Let $\mathbb{I} \longrightarrow G$ and $Q \longrightarrow G$ be the bundles, as in $(6.2 .3)$ (ii) and $(6.3 .1)$. Then we can consider that $I$ and $Q$ are embedded in $S^{2} \Sigma^{*}$ in such a way that

$$
\begin{gathered}
\mathbb{I}\left(\mathrm{V}_{2}\right)=\mathrm{H}^{\mathrm{O}}\left(\mathrm{P}\left(\sum\left(\mathrm{V}_{2}\right), \mathrm{O}\left(2-\mathrm{S}_{\mathrm{Q}\left(\mathrm{V}_{2}\right)}\right)\right),\right. \\
\mathrm{Q}\left(\mathrm{V}_{2}\right)=\mathrm{H}^{\circ}\left(\mathrm{P}\left(\sum\left(\mathrm{V}_{2}\right)\right), \mathrm{O}_{\mathrm{P}\left(\sum\left(\mathrm{V}_{2}\right)\right)}\right) \otimes \mathbb{C} \cdot \mathrm{Q},
\end{gathered}
$$

where $\nabla_{2} \in G(2, V)$; here $S_{P\left(\nabla_{2}\right)}=\sigma_{2,0}\left(P\left(\nabla_{2}\right)\right)$. W, $\nabla_{2} \in G$.

(6.3.6) Let now $C \subset X$ be a rational normal cubic curve. As we know ( see Corollary (5.3.2)), there exists a line $I \subset P^{4}=P(V)$ ( resp., a subspace $\nabla_{2} \subset V$, such that $\left.P\left(V_{2}\right)=I\right)$, such that $C_{1}=\sigma_{2,0}(I) \cdot X=$ $=c+\bar{C}(\bar{c}$ is the involutive of $C)$. But $C_{1}=$ $=S_{1} \cdot Q$, where $S_{1}, I=P\left(V_{2}\right)$, is as in the Corollary above. The last means that there exists a quadric $q$, which is a point of the space $\quad \mathbb{(}\left(\mathrm{V}_{2}\right) \oplus \mathrm{Q}\left(\mathrm{V}_{2}\right) \subset \mathrm{s}^{2} \Sigma^{*}\left(\nabla_{2}\right)$ and which splits, as a subvariety of $P\left(\sum\left(\nabla_{2}\right)\right)$. into 
two hyperplanes: $(q=0)=\langle c\rangle+\langle\bar{c}\rangle \subset P\left(\sum\left(v_{2}\right)\right)$. The last is equivaient, in the interpretation of the Corollary (6.3.5), to the existence of a quadric of rank $\leqslant 2$ in the vector-space "of quadrics" ( I $\oplus Q)\left(\nabla_{2}\right)$. UbviousIy, the opposite is aiso true : the existence of a quadric $q \in(\mathbb{I} \oplus Q)\left(V_{2}\right)$ of rank $\leqslant 2, q=\mathrm{H} . \overline{\mathrm{H}}$ means that the curves $C=S_{P\left(V_{2}\right)} \cdot P(H)$ and $C=S_{P\left(V_{2}\right)} \cdot P(\bar{H})$ are an involutive pair of rational normal cubics such that $\mathrm{C}_{\mathrm{P}\left(\mathrm{v}_{2}\right)}=\mathrm{C}+\overline{\mathrm{C}}$.

(6.3.7) CoRoliary. The factorfamily $l_{0}^{3}=\epsilon^{3} / \sigma$ (. see (5.3.2) ) is embedded naturally in the projectivized vector bundle $P_{G}(\mathbb{I} \oplus Q) \subset G(2, V) \times P\left(S^{2} \Sigma^{*}\right)$ as the set :

$$
\begin{aligned}
D_{2}=P\{\{ & \left(\nabla_{2}, q\right): \nabla_{2} \in G, q \in(\mathbb{I} \oplus Q)\left(\nabla_{2}\right) \subset s^{2} \Sigma^{*}\left(\nabla_{2}\right), \\
& , \operatorname{rank}(q) \leqslant 2\} .
\end{aligned}
$$

Postponing the comments of the Corollary till later on, we shall explain in brief some facts about the degeneration loci (determinantals) (see $[F$, ch. 14] ).

6.4. The factorfamily $\zeta_{0}^{3}$ as a determinantal. (6.4.1) DEFINITION.

(i) Let $\varphi: E \longrightarrow F$ be a homomorphism of vector bundies ( a vector bundle map) over the variety $Y$; let $\operatorname{rank} E=e, \operatorname{rank} F=f$. Let $k$ be a non-negative integer, such that $k \leqslant \min (e, f)$. The locus

$$
D_{\mathrm{K}}=D_{\mathrm{K}}(\varphi)=\{\mathrm{y} \in \mathrm{Y}: \operatorname{rank} \varphi(\mathrm{y}) \leqslant \mathrm{k}\} \subset \mathrm{Y}
$$

is called the $\mathrm{k}^{- \text {th }}$ degeneration locus ( the $\mathrm{k}^{- \text {th }}$ determinantal) of $\varphi$. 


\section{(ii) In particular, let $\bar{F}=E^{*} \otimes \bar{I}$ for some} invertible sheaf $I$ on $Y$. Nultipiyine by $I^{-1}$, we obtain tine map $\varphi \otimes I^{-1}: i \otimes I^{-1} \longrightarrow E^{*}$. Taking the dual $\left(Y \otimes I^{-1}\right)^{*}: E^{* *}=E \rightarrow E^{*} \otimes I$, we obtain another vector bundle map from $\bar{E}$ to $F=$ $=E^{*} \otimes I$. The vector bundle map $\varphi: I \longrightarrow E^{*} \otimes I$ is called symetric, if $\varphi$ is "selfdual", i. $\in$. if $\varphi=\left(\varphi \otimes I^{-1}\right)^{*}$. Using the natural correspondence between so defined symmetric maps and the "nets of quadrics" $\hat{\varphi}: I^{-1} \longrightarrow S^{2} B^{*}$, we define tre $K^{- \text {th }}$ degeneration locus (the $\mathbf{k}^{- \text {th }}$ determinantal) of the net of quadrics $\hat{\varphi}: I^{-1} \longrightarrow S^{2} E^{*}$ to be the $k^{-t h}$ determinantal of the corresponaing (symmetric) vector bunde map

$$
\varphi: E \longrightarrow E^{*} \otimes I \text {. }
$$

(6.4.2) As we know, II $\oplus Q \simeq \tau_{3, V^{*}}^{*} \oplus O_{G(2, V)}$, that is $Z=P_{G(2, V)}\left(\tau_{3, \nabla^{*}}^{*} \oplus O_{G(2, V)}\right) \simeq$

$$
\begin{aligned}
& \simeq P_{G(2, V)}(\mathbb{I} \oplus Q) \quad(\text { see Corollary }(6.2 .3 .)) \cdot \\
& \quad \text { Let } \pi: Z=P_{G}\left(\tau_{3, \nabla^{*}}^{*} \oplus O_{G}\right) \longrightarrow G=G(2, V)
\end{aligned}
$$

be the natural projection and let

$$
\tilde{\tau}_{3, V^{*}}^{*} \oplus 0_{G(2, \nabla)} \stackrel{\sim}{\longrightarrow} \oplus Q \subset s^{2} \Sigma^{*}
$$

be the abovedescribed embeding of vector bundles. Taking the tauthological sequence for the projectivized vector bunde $\pi: Z=P_{G}\left(\tau_{3, V^{*}}^{*} \oplus O_{G}\right) \longrightarrow G(2, \nabla):$ $0 \longrightarrow 0_{\pi}(-1) \stackrel{j}{\longrightarrow} \pi^{*}\left(\tau_{3, V^{*}}^{*} 0_{G}\right) \longrightarrow R \longrightarrow 0$, we obtain the composition of natural embeddings : 


$$
0 \rightarrow 0_{\pi}(-1) \stackrel{j}{\longrightarrow} \pi^{*}\left(\tau_{3, \nabla^{*}}^{*} \oplus 0_{G}\right) \stackrel{i}{\longrightarrow} \pi^{*}\left(s^{2} \Sigma^{*}\right)
$$

of vector bunòles over $Z$.

(6.4.3) By the construction of the tauthological secuences, the protectivization of the embeding of the sheaf

$0_{\pi}(-1)$ (= the relative $O(-1)$, or the tauthological subbundle ) :

$$
P\left(0_{\pi}(-1)\right) \subset P\left(\pi^{*}\left(\tau_{3, \nabla^{*}}^{*} \oplus O_{G}\right)\right)
$$

represents the embedaing of the points of the fibers of $P_{G}\left(\tau_{3, V^{*}}^{*} \oplus O_{G}\right)$, regarded, in view of the isomorphism $\tilde{L}_{3, V^{*}}^{*} \oplus O_{G} \simeq \mathbb{I} \oplus Q$,as quadratic hypersurfaces in the corresponding projective spaces $P\left(\Sigma\left(V_{2}\right)\right)$, $V_{2} \in G(2, V)$, lifted to $Z$. From the last and from the Corollary $(6.3 .7)$ we obtain :

$(6.4 .4)$ PFOEOSITION. Tine factorfamily $\zeta_{0}^{3}=\zeta^{3} / \sigma$ is embedded naturally in $Z=P_{G(2, V)}\left(\tau_{3, V^{*}}^{*} \oplus O_{G(2, V)}\right)$ as a second determinantal of the net of quadrics: $0 \longrightarrow 0_{\pi}(-1) \stackrel{j}{\longrightarrow} \pi^{*}\left(\tau_{3, V^{*}}^{*} \oplus 0_{G(2, V)}\right) \stackrel{i}{\longrightarrow} \pi^{*}\left(S^{2} \Sigma^{*}\right)$; i.e. $b_{0}^{3} \simeq D_{2}(\hat{\varphi})$, where $\hat{\varphi}=i \cdot j \quad$ (see $(6.4 .2))$ 
$\S 7$. The families $\epsilon^{3}$ and $\zeta_{0}^{3}$ as zero-schemes.

7.1. We shail use the same symbol $q$ for tine quadratic form $q \in S^{2} \Sigma^{*}\left(V_{2}\right)$ and for the corresponding symmetric operator $q: \sum\left(\mathrm{V}_{2}\right) \longrightarrow \sum^{*}\left(\mathrm{~V}_{2}\right)$. Then Proposition $(\epsilon .4 .4)$ means that $\epsilon_{0}^{3}=\left\{\left(V_{2}, q\right) \in Z: \operatorname{rank}(q) \leqslant 2\right\}=$ $=D_{2}(\hat{\varphi})$, ( the $q$ in the pair is regarded as a form, the $q$ after the colon is regarded as an operator). The condition $\operatorname{rank}(q)=k$ means that $\operatorname{dim} \operatorname{Im}(q)=k$ ( Im (q) is a subspace of $\sum^{*}\left(\nabla_{2}\right)$ ).

Let $0 \leqslant k \leqslant 5=\operatorname{dim} \sum\left(\mathrm{V}_{2}\right)$ and let

$$
\begin{aligned}
\tilde{D}_{k}= & D_{k}(\hat{\varphi})= \\
=\left\{\left(z ; \sigma_{k}\right) \in G_{z}\left(k, \Sigma^{*}\right)\right. & : \operatorname{Im}(q) \subseteq U_{k} \text {, where } \\
& \left.\therefore z \ni z=\left(V_{2}, q\right)\right\}
\end{aligned}
$$

The natural projection $\pi_{k}:\left(z ; U_{k}\right) \longmapsto z$ provides a map: $\pi_{k}: \tilde{D}_{k} \longrightarrow D_{k}$, which is an isomorphism outside the locus $D_{k-1} \subset D_{k}, 1 \leqslant k \leqslant 5=$ $=\operatorname{dim}$ (fibers of $\Sigma$ ). The natural embedaing $\tilde{D}_{k} C G_{Z}\left(k, \Sigma^{*}\right)$ can be included in the commutative diag ram :

$(7.1 .1)$

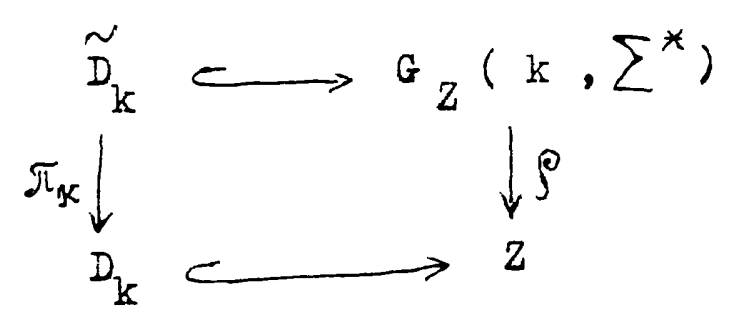

where $\rho: G_{Z}\left(k, \Sigma^{*}\right) \longrightarrow z$ is the natural projection. (7.1.2) RaVARK. Let $Q \in S^{2} \nabla_{8}^{*}(\operatorname{resp} .(Q:=0) \subset$ $C P\left(V_{8}\right)$ ) be a quadric. It is not hard to check that the . set of all the quadrics $Q$ in $P\left(V_{8}\right)$ ( resp. $Q \in S^{2} \nabla_{8}^{*}$ ), 
such that some of tine spaces $\prod_{2}\left(\nabla_{2}\right) \oplus Q\left(V_{2}\right)$ contains a cuadric of rark 1 ( a couble hyperplane in the corresponding $E\left(\sum\left(V_{2}\right)\right)\left(\right.$ resp., of $\left.s^{2} \sum^{*}\left(V_{2}\right)\right)$, has a coaimension $>1$ inside the space of quadrics in $P\left(\mathrm{~V}_{8}\right)$ ( resp., of $\left.S^{2} V_{8}^{*}\right)$. Therefore, for the general quaaric $Q$ in $\nabla_{8}$ the first determinantal $D_{1}(\hat{\varphi})$ vanishes. Because of that we can suppose in addition that the quadric $Q$ is choosen such that the open condition

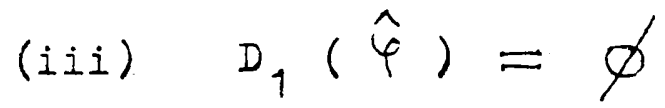

is fulfilled (see also the conditions (i) and (ii) in the oorollary $(6.3 .5)$ ).

Taking into account (iii) from the Remark, we obtain that the projection $\pi_{2}: \tilde{D}_{2} \longrightarrow D_{2}$ is an isomorphism.

7.2.

(7.2.1) Let

$$
0 \longrightarrow \tau_{2, \Sigma^{*}} \stackrel{\alpha}{\longrightarrow} G_{2}\left(2, \Sigma^{*}\right) \times \Sigma^{*} \stackrel{\beta}{\rightarrow} \tau_{3, \Sigma}^{*} \longrightarrow 0
$$

be the standard tauthological sequence on the grassmannization $\rho: G_{z}\left(2, \Sigma^{*}\right) \longrightarrow z$. The embedding $\alpha: \tau_{2, \Sigma^{*}} \hookrightarrow G_{z}\left(2, \Sigma^{*}\right) \times \sum^{*}$ defines an embeding $s^{2} \alpha: s^{2} \tau_{2, \Sigma^{*}} \longleftrightarrow G_{Z}\left(2, \Sigma^{*}\right) \times s^{2} \Sigma^{*}$. The embedding $s^{2} 2$ can be included in the exact sequence:

$$
0 \rightarrow s^{2} \tau_{2, \Sigma^{*}} \stackrel{s^{2} \alpha}{\longrightarrow} G_{2}\left(2, \Sigma^{*}\right) \times s^{2} \Sigma^{*} \stackrel{\gamma^{2}}{\rightarrow} \operatorname{Coker}(2) \longrightarrow 0 \text {, }
$$

where Coker(2) is the factor-bundle $S^{2} \Sigma^{*} / s^{2} \alpha\left(s^{2} \tau_{2, \Sigma^{*}}\right)$ on $G_{Z}\left(2, \Sigma^{*}\right)$.

(7.2.2) On the other hana, the net of quadrics $\hat{\varphi}$ de- 
fines the embeding $0 \rightarrow 0_{\pi}(-1) \stackrel{\hat{\varphi}}{\longrightarrow} S^{2} \Sigma^{*}$ of bundies over $Z$. Taking into account the projection

$\rho: G_{Z}\left(2, \Sigma^{*}\right) \longrightarrow Z$ we car jook at the sheaves $c_{\pi}(-1)$ and $\sum$ as sheaves on $G_{Z}\left(2, \Sigma^{*}\right)$ (here we omit, for simplicity, the symbol $\rho^{*}$ ).

Composing the morpinisms in the sequences above, we obtain the sequence

$$
0 \rightarrow 0_{\pi}(-1) \stackrel{\hat{\varphi}}{\longrightarrow} G_{2}\left(2, \Sigma^{*}\right) \times s^{2} \Sigma^{*} \stackrel{\gamma}{\longrightarrow} \text { Coker }
$$

of natural maps of sheaves (resp., of bundles) over $G_{z}\left(2, \Sigma^{*}\right)$. Multiplying by the sheaf $\left(0_{\pi}(-1)\right)^{*}=$ $=0_{\pi}(1)$, we obtain the composition :

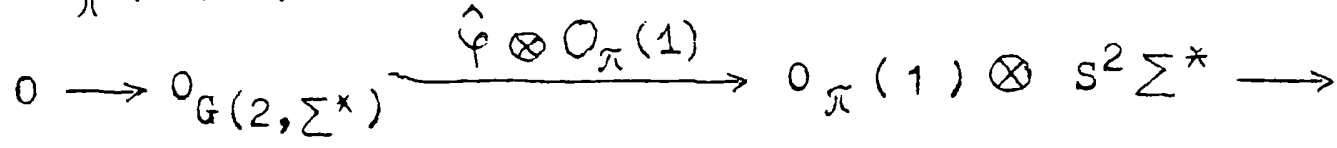

$$
\begin{aligned}
& \stackrel{\gamma \otimes O_{\pi}(1)}{\longrightarrow} O_{\pi}(1) \otimes \text { Coker (2) ; }
\end{aligned}
$$

the last defines a section $s_{0}$ of the sheaf $O_{\pi}(1) \otimes$ Coker (2) over the variety: $G_{Z}\left(2, \Sigma^{*}\right)$, $s_{0}=\left(\gamma \otimes 0_{\pi}(1)\right) \cdot\left(\hat{\varphi} \otimes 0_{\pi}(1)\right)$.

(7.2.3) By definition the zero scheme $2\left(s_{0}\right) \subset G_{Z}\left(2, \Sigma^{*}\right)$ of the section $s_{0} \in H^{a}\left(G_{Z}\left(2, \Sigma^{*}\right), O_{\pi}(1) \otimes \operatorname{Coker}(2)\right)$ coincides with the $0^{- \text {th }}$ determinantal $D_{0}\left(s_{0}\right)$ of the section $s_{0}$, regarded as a vector bundle map

$$
\begin{aligned}
& s_{0}: O_{G}\left(2, \Sigma^{*}\right) \longrightarrow O_{\pi}(1) \otimes \text { Coker (2). Consequently, } \\
& z\left(s_{0}\right)=D_{0}\left(s_{0}\right)= \\
& \quad=\left\{\left(z ; U_{2}\right) \in Z \times G\left(2, \Sigma^{*}\left(V_{2}\right)\right): z=\left(V_{2}, q\right) \& s_{0}\left(z ; U_{2}\right)=0\right\}
\end{aligned}
$$

So we have:

(1) $\hat{\varphi}=i \cdot j$, where the embeàing $j: O_{\pi}(-1)$ 
$\longrightarrow \pi^{*}\left(\tau_{3, \nabla^{*}}^{*} \oplus O_{G(2, V)}\right)$ represents the closed embedaing of the"point" $q$ in the fiber $\pi^{-1}\left(V_{2}\right) \subset z$ and the embeding $i: \pi^{*}\left(\tau_{3, V^{*}}^{*} \oplus O_{G(2, \nabla)}\right) \circlearrowright s^{2} \Sigma^{*}$ corresponas to the natural representation of the points in the fibers of $\tau_{3, V^{*}}^{*} \oplus O_{G(2, V)}$ as quaürics in the fibers $\sum\left(v_{2}\right)$ of $\sum$ (here we use the isomorphism $\tau_{3, \nabla^{*}}^{*} \oplus \mathrm{O}_{G(2, V)} \stackrel{\sim}{\longrightarrow} \mathbb{I} \oplus Q$, see $(6.2 .3)$ (ii) and $(6.3 .1))$;

\section{(2) The maps}

$$
\gamma(z)=\gamma\left(v_{2}, q\right): s^{2} \Sigma^{*}\left(v_{2}, q ; U_{2}\right) \longrightarrow \text { Coker (2), }
$$

as factor-maps, send the quadrics $q \in S^{2} \Sigma^{*}\left(V_{2}\right)$ to the corresponding classes $q\left(\bmod . S^{2} U_{2}\right)$, over the elements $\left(z ; U_{2}\right)=\left(V_{2}, q ; U_{2}\right) \in G_{Z}\left(2, \Sigma^{*}\right)$.

From (1) and (2) we derive, that

$Z\left(s_{0}\right)=\left\{\left(\nabla_{2}, q ; \sigma_{2}\right) \in G_{Z}\left(2, \Sigma^{*}\right): q\left(\bmod \cdot s^{2} U_{2}\right)=0\right.$; $q$ regarảed as an element of $\left.s^{2} \Sigma^{*}\left(\nabla_{2}\right)\right\}=$ $=\left\{\left(\mathrm{V}_{2}, \mathrm{q} ; \mathrm{U}_{2}\right) \in G_{2}\left(2, \Sigma^{*}\right): \mathrm{U}_{2} \subseteq\right.$ Image $(\mathrm{q}) \subset \Sigma^{*}\left(\mathrm{~V}_{2}\right)$; $q$ regarded as an operator $\left.q \in \operatorname{Sym}\left(\Sigma\left(\mathrm{v}_{2}\right), \Sigma^{*}\left(\mathrm{~V}_{2}\right)\right)\right\}$. Consequently, $z\left(s_{0}\right)=\widetilde{D}_{2}(\widehat{\varphi}) \subset G_{Z}\left(2, \Sigma^{*}\right)$, ( see 7.1 and $(7.1 .1)$ ) . But, by the condition (iii) (see Remark (7.1.2)), $D_{1}(\hat{\varphi})=\varnothing$, that is, there are no double hyperplanes in the fibers $\mathrm{H}^{\mathrm{O}}\left(\mathrm{P}\left(\sum\left(\mathrm{V}_{2}\right)\right), \mathrm{O}\left(2-\sigma_{2,0}\left(\mathrm{P}\left(\mathrm{V}_{2}\right)\right) . \mathrm{X}_{10}\right)\right)$, $V_{2} \in G(2, V)$ of the bundle "of. quadrics" $\mathbb{I} \oplus Q$. 
Consequently, the natural projection $\pi_{2}: \tilde{D}_{2}(\hat{\varphi}) \longrightarrow$ $\longrightarrow D_{2}(\hat{\varphi}) \quad($ cf. $(7.1 .2))$ is an isomorphism. Summing up the obtained incidences, we derive: (7.2.4) COROLIARY. Let $\mathrm{v}_{8} \subset \mathrm{v}_{10}=\wedge^{2} \mathrm{v}$ and $\mathrm{Q}$ fulfil the open conditions (i), (ii) from the corollary $(6.3 .5)$ and (iii) from the Kemarix (7.1.2). Iet $l_{0}^{3}=$ $=C^{3} / \sigma$ be the factorfamily of the family of rational normal cubics on $X=X_{10}=G(2, V) \cap P\left(V_{8}\right) \cap Q$ and let $s_{0} \in \mathrm{H}^{0}\left(G_{Z}\left(2, \Sigma^{*}\right), 0_{\pi}(1) \otimes\right.$ Coker (2)) be the section, described in (7.2.2). Then $\epsilon_{0}^{3}$ embeds naturally in $G_{Z}\left(2, \Sigma^{*}\right)$ as a zero scheme of the section $s_{0}$, that is:

$$
\zeta_{0}^{3} \sim 2\left(s_{0}\right) \subset G_{Z}\left(2, \Sigma^{*}\right) \text {. }
$$

(7.2.5) Nons. By convention, the symbol $\rho^{x}$, where $\rho: G_{Z}\left(2, \Sigma^{*}\right) \longrightarrow z$, is dropped, i.e. with some abuse of the notations we write $\rho^{*} O_{\pi}(1) \otimes$ Coker $(2)=$ $=0_{\pi}(1) \otimes$ Coker (2).

There exists a natural isomorphism of duality :

$$
\delta: G_{Z}\left(2, \Sigma^{*}\right) \sim G_{Z}(3, \Sigma) \text {, }
$$

induced by the natural duality of the fibers

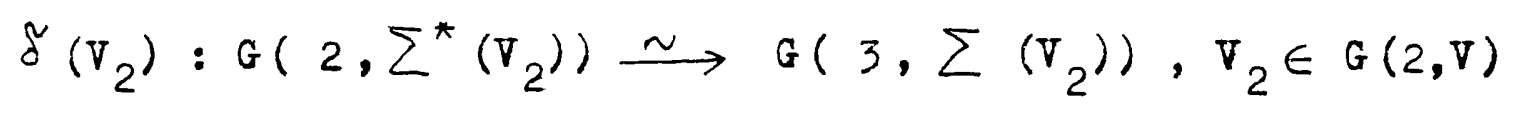

The composition $s_{0}^{*}=\delta \cdot s_{0}$ defines a section of (the isomorphic $\delta$-preimage of ) the sheaf $O_{\pi}(1) \otimes$ Coker (2) on $G_{Z}(3, \Sigma)$. So we obtained :

(7.2.6) COROILARY. In the conditions of the Corollary (7.2.4) there is a natural embedding $\zeta_{0}^{3} \hookrightarrow_{Z}(3, \Sigma)$, such that : 
(1) $\varphi_{0}^{3} \stackrel{\sim}{\longrightarrow}\left(s_{0}^{*}\right) \subset G_{Z}(3, \Sigma)$, where $s_{0}^{*}=\delta \cdot s_{0}$ is the section, defined just above;

(2) Let $(\mathrm{C}, \overline{\mathrm{C}})$ be an element of $\ell_{0}^{3}$ (i.e. (C, $\bar{c}$ ) is a pair of involutive rational normal cubics on $X=x_{10}$ ). Let $v_{2} \subset \mathrm{V}$ be the subspace ( of aim. $=2$ ), such that $C_{P\left(V_{2}\right)}=\sigma_{2,0}\left(P\left(\nabla_{2}\right)\right) \cdot X_{10}=C+\bar{C}$ ( see Corollary $(5.3 .2)$ ) and let $\mathrm{E}_{\mathrm{C}}$ and $\mathrm{H}_{\bar{C}} \in \Sigma^{*}\left(\mathrm{~V}_{2}\right)$ be the hyperplanes in $\sum\left(\mathrm{V}_{2}\right)$ such that $\langle\mathrm{C}\rangle=\operatorname{Span}(\mathrm{C})=\mathrm{P}\left(\mathrm{H}_{\mathrm{C}}\right)$ and $\langle\bar{C}\rangle=\operatorname{Span}(\bar{C})=P\left(H_{\bar{C}}\right)$. Then the embedding $\ell_{0}^{3} C G_{Z}(3, \Sigma)$ sends the pair $(C, \bar{C})$ to the triple $\left(\mathrm{V}_{2}, \mathrm{q}=\mathrm{H}_{\mathrm{C}} \cdot \mathrm{H}_{\overline{\mathrm{C}}} ; \mathrm{H}_{\mathrm{C}} \cap \mathrm{H}_{\overline{\mathrm{C}}}\right) \in\left(\mathrm{V}_{2}, \mathrm{q}=\mathrm{H}_{\mathrm{C}} \cdot \mathrm{H}_{\overline{\mathrm{C}}}\right) \times \mathrm{G}\left(3, \sum\left(\mathrm{V}_{2}\right)\right)$ $\subset G_{2}(3, \Sigma)$.

Proof. Point (1) follows immediately from the definition of the section $\mathbf{s}_{0}^{*}=\delta \cdot \mathbf{s}_{0}$ and from the previous Corollary. To prove (2) it is sufficient to see that the thira member $\mathrm{H}_{\mathrm{C}} \cap \mathrm{H}_{\overline{\mathrm{C}}}$ of the triple corresponds, by the duality $\delta$, to the subspace $U_{2}:=\operatorname{Image}(q) \subset \Sigma^{*}\left(\mathrm{~V}_{2}\right)$, where

$$
q=\mathrm{H}_{\mathrm{C}} \cdot \mathrm{H}_{\overline{\mathrm{C}}} \text { is regarded as an operator from }
$$

$\operatorname{Sym}\left(\Sigma\left(\nabla_{2}\right), \Sigma^{*}\left(\nabla_{2}\right)\right)$. Moreover, $\operatorname{dim}\left(\operatorname{Image}\left(q=\mathrm{H}_{\mathrm{C}} \cdot \mathrm{H}_{\bar{C}}\right)\right)=$ $=2$, because of the fact, that $D_{1}(\hat{\varphi})=\varnothing$. In particular, $\mathrm{H}_{\mathrm{C}}$ and $\mathrm{H}_{\overline{\mathrm{C}}}$ are not proportional, that is

$$
\begin{aligned}
& U_{2}=\text { Image }\left(q=H_{C} \cdot H_{\bar{C}}\right) \text { belongs to the Grassmannian } \\
& G\left(2, \Sigma\left(V_{2}\right)\right) \text {. }
\end{aligned}
$$




\section{3.}

$(7.3 .1)$ Let

$0 \longrightarrow \tau_{4, \Sigma} \longrightarrow P_{2}\left(\Sigma^{*}\right) \times \Sigma \longrightarrow \tau_{1, \Sigma^{*}} \longrightarrow 0$

be the standard tauthological sequence on the projectivization $P_{Z}\left(\Sigma^{*}\right)=G_{Z}(4, \Sigma)$. Taking the dual, we obtain the natural surjection $\mathrm{P}_{\mathbf{Z}}\left(\Sigma^{*}\right) \times \Sigma^{*} \rightarrow \tau_{4, \Sigma}^{*} \longrightarrow 0$, hence, the surjection

$$
\varepsilon:{ }_{Z}\left(\Sigma^{*}\right) \times s^{2} \Sigma^{*} \longrightarrow s^{2} \tau_{4, \Sigma}^{*} \longrightarrow 0 \text {. }
$$

Let now $\quad \tau: P_{Z}\left(\Sigma^{*}\right) \longrightarrow z$ be the natural projection. Taking the $\tau$-preimages of the sheaves, we lift the map $\hat{\varphi}$ onto $\mathrm{P}_{2}\left(\Sigma^{*}\right)$ :

$$
0 \rightarrow 0_{\pi}(-1) \stackrel{\hat{\varphi}}{\longrightarrow} \mathrm{P}_{\mathrm{Z}}\left(\Sigma^{*}\right) \times \mathrm{S}^{2} \Sigma^{*} \text {; }
$$

as usual the symbol $\tau^{*}$ is dropped.

$$
\text { Just as in }(7.2 .2) \text {, after multiplying by } 0_{\pi}(1) \text {, we }
$$
obtain the composite map :

$$
\begin{aligned}
& 0 \rightarrow O_{P_{Z}}\left(\Sigma^{*}\right) \stackrel{\hat{\varphi} \otimes O_{\pi}(1)}{\longrightarrow} P_{Z}\left(\Sigma^{*}\right) \times O_{\pi}(1) \otimes s^{2} \Sigma^{*} \longrightarrow \\
& \stackrel{\varepsilon \otimes O_{\pi}(1)}{\longrightarrow} 0_{\pi}(1) \otimes s^{2} \tau_{4, \Sigma}^{*},
\end{aligned}
$$

hence, we obtain a well-defined section $\mathbf{s}$ of the sheaf

$$
\begin{aligned}
& o_{\pi}(1) \otimes s^{2} \tau_{4, \Sigma}^{*} \text { over } P_{z}\left(\Sigma^{*}\right), s= \\
& =\left(\varepsilon \otimes o_{\pi}(1)\right) \cdot\left(\hat{\varphi} \otimes o_{\pi}(1)\right) .
\end{aligned}
$$

As in $\S 7.2$ we shall prove the following

(7.3.2) PROPOSITION. In the conditions of the Corollary (7.2.4), the family $\zeta^{3}$ of the rational nomal cubics on $X$ is embedded naturally in the projectivized bundle $P_{Z}\left(\Sigma^{*}\right)$ as a zero scheme of the section $s$, that is :

$$
\ell^{3} \stackrel{\sim}{\longrightarrow}(\mathrm{s}) \subset \mathrm{P}_{\mathrm{z}}\left(\Sigma^{*}\right) \text {. }
$$


Proaf. ( for more details see the proof of the Corollary $(7.2 .4))$. As in (7.2.3) by the definition of the tauthological sequence the map. $\varepsilon: P_{Z}\left(\Sigma^{*}\right) \times s^{2} \Sigma^{*} \longrightarrow s^{2} \tau_{4, \Sigma}^{*}$ sends the element $\left(\nabla_{2}, q ; Y_{4}\right)$ of the fibre $\tau^{-1}\left(V_{2}, q\right)$ to the element $\left(V_{2}, q ;\left.q\right|_{Y_{4}}\right) \in s^{2} \tau_{4, \Sigma}^{*}$ (here $\left.\mathrm{q}\right|_{\mathrm{Y}_{4}}$ is the restriction of the quadric $q \in \mathrm{S}^{2} \Sigma^{*}\left(\mathrm{v}_{2}\right)$ to the subspace $\left.Y_{4} \subset \sum\left(V_{2}\right), \operatorname{dim}\left(Y_{4}\right)=4\right)$. Consequently, the elements of the zero scheme of $s$ are defined by the condition: $Y_{4} \subseteq \operatorname{Kernel}(q)$; here the quadric $q$ is regarded as an operator from $\operatorname{Sym}\left(\sum\left(\mathrm{V}_{2}\right), \Sigma^{*}\left(\mathrm{~V}_{2}\right)\right)$. In other words, the zero scheme

$$
Z(s)=\left\{\left(V_{2}, q ; Y_{4}\right):\left(V_{2}, q\right) \in Z, Y_{4} \subset \sum\left(V_{2}\right)\right.
$$
is an element of $G\left(4, \sum\left(V_{2}\right)\right)$ such that $Y_{4} \leqslant \operatorname{KerneI}(q)$; But the symetric operator $q: \sum\left(\mathrm{V}_{2}\right) \longrightarrow \sum^{*}\left(\mathrm{~V}_{2}\right)$ : $\operatorname{dim} \sum\left(v_{2}\right)=5$, contains four-dimensional subspaces in its kernel, if and only if $\operatorname{rank}(q) \leqslant 2$. By the way, there are no quadrics $q$ of rank $=1$, since $D_{1}(\hat{\varphi})=\varnothing \quad$ ( see for example (7.2.3) - the proof of the Corollary (7.2.4)). Because of that $\operatorname{rank}(q) \leqslant 2$ iff $\operatorname{rank}(Q)=2$, that is iff $q=H . \bar{H}$, for some non-proportional linear functions ( non-coinciaent hyperplanes $) \mathrm{H}$ and $\overline{\mathrm{H}}$ of $\Sigma^{*}\left(\mathrm{~V}_{2}\right)$. But the projective hyperplanes $P(\mathrm{H})$ and $P(\bar{H})$ cut out on $\mathrm{X}$ ( in the described situation) a pair of involutive rational normal cubics $C=X \cap P(H)$ and $\bar{C}=X \cap P(\bar{H})$. Because of that the zero scheme $Z(s)$ 
describes the set of the components of the quadrics $q$ of rank $=2, q$ being the second element of the pair $\left(\nabla_{2}, q\right) \in D_{2}(\hat{\varphi}) \quad($ see Froposition (6.4.4), \& 7.1 and $\S 7.2)$. Equivalently, the zero scheme $Z(s)$ describes the set of the rational normal cubics ( the intersections of $X=X_{10}$ with the corresponaing components $P(H)$ and $P(\bar{H})$ of the quadrics $q=H \cdot \bar{H}$ of rank $=2$, as above) . The Proposition is proved. From the proof of the Proposition (7.3.2), just as for the Corollary (7.2.6) (2), we obtain the following geometrical description of the embedding $\zeta^{3} \hookrightarrow \mathrm{P}_{Z}\left(\Sigma^{*}\right)=$ $=G_{Z}(4, \Sigma):$

(7.3.3) cOROLIARY. Let $c \in \zeta^{3}$ be a rational normal cubic curve on $X=x_{10}$. Let $V_{2} \subset V$ be the subspace ( of dime $=2$ ), such that $C_{P\left(V_{2}\right)}=$ $=\sigma_{2,0}\left(P\left(V_{2}\right)\right) \cdot X=C+\bar{C}$, where $\bar{C}$ is the involutive of C (see Corollary (5.3.2) ) and let $\mathrm{H}_{\mathrm{C}}$ and $\mathrm{H}_{\overline{\mathrm{C}}} \in \Sigma^{*}\left(\mathrm{~V}_{2}\right)$ be the hyperplanes in $\sum\left(\mathrm{V}_{2}\right)$, such that $\langle\mathrm{C}\rangle=\operatorname{Span}(\mathrm{C})=\mathrm{P}\left(\mathrm{H}_{\mathrm{C}}\right)$. and $\langle\bar{C}\rangle=$ $=\operatorname{Span}(\bar{C})=P\left(\bar{H}_{\bar{B}}\right) \cdot$ Then the embedding $\varphi^{3} \longrightarrow \mathrm{P}_{\mathrm{Z}}\left(\Sigma^{*}\right)=\mathrm{G}_{\mathrm{Z}}(4, \Sigma)$ sends the element $c \in G^{3}$ to the triple $\left(v_{2}, q=H_{C} \cdot H_{\bar{C}} ; H_{C}\right) \epsilon$ $\epsilon\left(\mathrm{V}_{2}, \mathrm{q}=\mathrm{H}_{\mathrm{C}} \cdot \mathrm{H}_{\overline{\mathrm{C}}}\right) \times \mathrm{G}\left(4, \Sigma\left(\mathrm{V}_{2}\right)\right) \subset \mathrm{G}_{\mathrm{Z}}(4, \Sigma)=$ $=P_{Z}\left(\Sigma^{*}\right)$. 
§ 8. Tangent Bundle Theorem for the family of rational normal cubics $l^{3}$.

8.1. Before formulating and proving the Tangent Bundie Theorem (T.B.T.), we shall carry out some constructions, which clarify the local situation. From now on we shall suppose that the curve $c \in \ell^{3}$ is choosen sufficiently general, in particular, we can suppose that the curve $C$ and its involutive $\bar{C}$ are smooth rational normal cubics with normal bundies $N_{C} / X$ and $N_{\bar{C} / X}$, isomorphic to $0_{P} \oplus 0_{P} 1(1)$ (see Proposition 5.1), etc.

(8.1.1) Let $C$ and $\bar{C}$ be a pair of involutive raticnal normal cubics on $\mathrm{X}$, let $\mathrm{V}_{2} \subset \mathrm{V}$ be the corresponding subspace of dim. $=2$, such that $\mathrm{C}_{1}=$ $=C+\bar{C}$, where $I=P\left(\nabla_{2}\right)$ ( see Corollary (5.3.2)). Let $W=G \cdot P\left(V_{8}\right), X=X_{10}=W \cdot Q=G \cdot P\left(\nabla_{8}\right) \cdot Q$ and $s_{1}=\sigma_{2,0}(I) . W$ are defined as before.

Examine the pairs of successive embeddings:

$$
c \subset \mathbf{x} \subset \mathrm{W} \text { and } \mathrm{C} \subset \mathrm{S}_{1} \subset \mathrm{W}
$$

and the corresponding exact sequences of normal sheaves:
(1)
(2)
$\mathrm{O} \rightarrow \mathrm{N}_{\mathrm{C} / \mathrm{X}} \longrightarrow \mathrm{N}_{\mathrm{C} / \mathrm{W}} \longrightarrow \mathrm{N}_{\mathrm{X} / \mathrm{W}} \otimes \mathrm{O}_{\mathrm{C}} \longrightarrow 0$ and

$$
0 \rightarrow \mathrm{N}_{\mathrm{C} / \mathrm{S}_{I}} \rightarrow \mathrm{N}_{\mathrm{C} / \mathrm{W}} \longrightarrow \mathrm{N}_{\mathrm{S}_{I} / \mathrm{W}} \otimes \mathrm{O}_{\mathrm{C}} \longrightarrow 0 \text {. }
$$

Multiplying the sequences (1) and (2) by the sheaf $O(-1)=O_{P\left(V_{8}\right)}(-1)$ and identifying the middle members, we obtain the diagonal map:

(3) $\quad \varphi_{\mathrm{C}}: \mathbb{N}_{\mathrm{C} / \mathrm{S}_{I}} \otimes O(-1) \rightarrow \mathrm{N}_{\mathrm{C} / \mathrm{W}} \otimes O(-1) \rightarrow \mathrm{N}_{\mathrm{X} / \mathrm{W}} \otimes O(-1) \otimes O_{C}$ Since $\operatorname{deg}(C)=3$, then $0(-1) \otimes 0_{C} \simeq 0_{C}(-3)$, $C \simeq \mathrm{P}^{1}$. 
From $X=W \cdot Q$, where $Q$ is a quadric in $P^{7}=P\left(V_{8}\right)$, it follows that $I_{X / W}=O_{X}(2)$. Therefore,

$N_{X / W} \otimes O(-1) \otimes O_{C} \simeq O(1) \otimes O_{C} \simeq O_{C}(3)$.

(8.1.2) On the other hand, $N_{C} / S_{I}=0(1) \otimes 0_{C}$, since $C$ is a hyperplane section of the rat. norm. cubic scroll $S_{1} \subset \operatorname{span}\left(S_{1}\right)=P\left(\Sigma\left(V_{2}\right)\right), I=P\left(V_{2}\right) ;($ see $(5.4 .2))$. Therefore :

(3:). $\quad \varphi_{C}: O_{C} \longrightarrow O(1) \otimes O_{C}=o_{C}(3)$.

Taking into account the identifications above, the sequence

$(2) \otimes O(-1)$ takes the form:

(2'). $\quad \mathrm{O} \rightarrow \mathrm{O}_{\mathrm{C}} \rightarrow \mathrm{N}_{\mathrm{C} / \mathrm{W}} \otimes \mathrm{O}(-1) \longrightarrow \mathrm{N}_{\mathrm{S}_{1} / \mathrm{W}} \otimes \mathrm{O}(-1) \otimes \mathrm{O}_{\mathrm{C}} \rightarrow \mathrm{C}$ (8.1.3) IEMTA.

Let $c \in l^{3}(x)$ be a general $r$. n. cubic. Then $\mathrm{N}_{\mathrm{S}_{I} / \mathrm{W}} \otimes \mathrm{O}_{\mathrm{C}} \simeq \mathrm{O}_{\mathrm{C}}(2) \oplus \mathrm{O}_{\mathrm{C}}(2)$.

The proof of the lemma will be given later on (see $(8.1 .10))$.

(8.1.4) From (8.1.3) we derive the identification:

(4) $\quad N_{S_{I} / W} \otimes O(-1) \otimes O_{C} \simeq O_{C}(-1) \oplus O_{C}(-1)$, therefore the long exact sequence of cohomologies, associated to the sequence ( $\left.2^{\prime}\right)$, defines the natural isomorphisms :

(5) $\quad \mathrm{H}^{\mathrm{i}}\left(\mathrm{C}, \mathrm{O}_{\mathrm{C}}\right) \stackrel{\sim}{\longrightarrow} \mathrm{H}^{i}\left(C, \mathrm{~N}_{\mathrm{C} / \mathrm{W}} \otimes \mathrm{O}(-1)\right), i=0,1, \ldots$

In fact, all the cohomology groups of the sheaf $\mathrm{N}_{\mathrm{S}_{I} / \mathrm{W}} \otimes O(-1) \otimes \mathrm{O}_{\mathrm{C}} \quad$ vanish.

(8.1.5) Let now look at the sequence (1) $\otimes O(-1)$. Since $\quad N_{C / X} \otimes O(-1) \simeq O_{C}(-3) \oplus O_{C}(-2)$, then $\mathrm{H}^{0}\left(C, \mathrm{~N}_{\mathrm{C} / \mathrm{X}} \otimes O(-1)\right)=0$. 
From $\quad H^{1}\left(C, N_{C / X}\right)=H^{1}\left(C, O_{C} \oplus O_{C}(1)\right)=0$, we obtain that the tangent space $T_{\mathrm{G}^{3}}{ }^{(\mathrm{C})}$ is isomorphic to $\mathrm{H}^{\mathrm{O}}\left(\mathrm{C}, \mathrm{N}_{\mathrm{C} / \mathrm{X}}\right)$.

Let $\Re_{l^{3}}=T_{l^{3}}^{*}$ be tine cotangent sheaf of

and let $\omega_{X}$ be the sheaf of hinger differential forms on $\mathrm{X}$ ( the canonical sheaf of $\mathrm{X}$ ). Using the Serre duality, we can write the following sequence of identities:

$$
\begin{aligned}
& \mathbb{H}_{C^{3}(C)=} T_{C^{3}(C)=H^{\circ}\left(C, N_{C / X}\right)^{*}=}^{H^{*}}= \\
= & H^{-1}\left(C, N_{C / X}^{*} \otimes W_{C}\right)=H^{1}\left(C, N_{C / X} \otimes \operatorname{det} \mathbb{N}_{C / X}^{*} \otimes \omega_{C}\right)= \\
= & H^{1}\left(C, N_{C / X} \otimes O_{C}(-1) \otimes \omega_{C}\right)=H^{1}\left(C, N_{C / X} \otimes O(-1)\right)= \\
= & H^{1}\left(C, N_{C / X} \otimes \omega_{X}\right), \text { since } \quad \omega_{X}=O(-1) \otimes O_{X} .
\end{aligned}
$$

From the last we obtain that the long exact cohomology sequence, associated to the exact sequence $(1) \otimes O(-1)$ takes the form:

$$
\begin{aligned}
& \text { (1') } \quad 0 \longrightarrow\left(\mathrm{H}^{0}\left(\mathrm{C}, \mathrm{N}_{\mathrm{C} / \mathrm{X}} \otimes \omega_{\mathrm{X}}\right)=0\right) \rightarrow \mathrm{H}^{0}\left(\mathrm{C}, \mathrm{N}_{\mathrm{C} / \mathrm{W}} \otimes O(-1)\right) \rightarrow \\
& \longrightarrow H^{\circ}\left(C, O(1) \otimes O_{C}\right) \longrightarrow\left(H^{1}\left(C, N_{C / X} \otimes \omega_{X}\right)=\mathscr{H}_{G^{3}}(c)\right)- \\
& \longrightarrow \mathrm{H}^{1}\left(\mathrm{C}, \mathrm{N}_{\mathrm{C} / \mathrm{W}} \otimes \mathrm{O}(-1)\right) \longrightarrow \quad \cdots \quad \text {. }
\end{aligned}
$$

Using (5) we obtain the sequence ( see also (8.1.2) ( $\left.3^{\prime}\right)$ ): (1") $0 \longrightarrow \mathrm{H}^{\circ}\left(\mathrm{C}, \mathrm{O}_{\mathrm{C}}\right) \stackrel{\mathrm{H}^{\circ}\left(\varphi_{C}\right)}{\longrightarrow} \mathrm{H}^{\circ}\left(\mathrm{C}, \mathrm{O}(1) \otimes \mathrm{O}_{\mathrm{C}}\right) \longrightarrow$ $\longrightarrow \mathscr{P}_{\mathrm{e}^{3}}(\mathrm{C}) \longrightarrow\left(\mathrm{H}^{1}\left(\mathrm{C}, \mathrm{O}_{\mathrm{C}}\right)=0\right)$

(8.1.6) If we suppose for a while that we have performed a globalization of the constructions above, simultaneously for all the elements $C$ of the family of rational normal cubics $l^{3}=C^{3}(x)$, then the members of the secuence 
(11) will be induced by restriction on the fibers from sheaves, cefined globally on the family $t^{3}$ ( as the fiber $\left.\oint_{e^{3}}(c)\right)$.

(o) kavinur. In fact, the aiagonal map $\varphi_{C}: O_{C} \longrightarrow O(1) \otimes O_{C}$ (see (3')) corresponds to. the choice of the divisor $\mathrm{C} \cap \overline{\mathrm{C}}$ on $\mathrm{C}$; the last divisor represents the hyperplane section of $\mathrm{C}$ in the projective space $\langle\mathrm{C}\rangle=$ $=\operatorname{span}(C) \subset P^{3}(\operatorname{see}(5.4 .1)$ and $(5.4 .2))$. (8.1.7) On the other hand, as we know, the family $\mathrm{l}^{3}$ is embedded in $P_{Z}\left(\Sigma^{*}\right)=G_{Z}(4, \Sigma)$ as a zero scheme of the section $\mathbf{s}$, and the factorfamily $\zeta_{0}^{3}=\zeta^{3} / \sigma$ is embedied in $G_{Z}(3, \Sigma)$ as a zero scheme of the section $s_{0}^{*}($ see Cor. $(7.2 .6)$, Prop. $(7.3 .2)$ and Cor. $(\% 3.3))$. From the last we conclude, that the tauthological sheaves $\tau_{3, \Sigma}$ and $\tau_{2, \Sigma^{*}}^{*}$ from the stanciard tauthological sequence :

(a) $0 \longrightarrow \tau_{3, \Sigma} \longrightarrow G_{2}(3, \Sigma) \times \Sigma \longrightarrow \tau_{2, \Sigma^{*}}^{*} \longrightarrow 0$ are well-defined on the family $\zeta_{0}^{3}$, and the tauthological sheaves $\tau_{4, \Sigma}$ and $\tau_{1, \Sigma^{*}}^{*}$ from the sequence: (b) $0 \longrightarrow \tau_{4, \Sigma} \longrightarrow \mathrm{P}_{2}\left(\Sigma^{*}\right) \times \Sigma \longrightarrow \tau_{1, \Sigma}^{*} \longrightarrow 0$ are well-defined on the family $e^{3}$.

But the existence of the natural 2-sheeted covering $\pi: \zeta^{3} \longrightarrow l_{0}^{3}$, induced from the involution $\sigma: \zeta^{3} \longrightarrow \zeta^{3}\left(\sigma(c)=\bar{c}, \sigma^{2}=\right.$ id $)$ shows that the $(\pi$-preimages of the $)$ sheaves $\tau_{3, \Sigma}$ and $\tau_{2, \Sigma^{*}}^{*}$ are defined also on the family $\ell^{3}$; 
as usual we write $\tau_{3, \Sigma}$ and $\tau_{2, \Sigma^{*}}^{*}$ instead of $\pi^{*} \tau_{3, \Sigma}$ and $\pi^{*} \tau_{2, \Sigma^{*}}$. Then, the (lifted by $\pi$ ) exact sequence (a) on, together with the exact sequence (b), define an embedaing j: $\tau_{3, \Sigma} \subset \tau_{4, \Sigma}$ of sheaves on $\zeta^{3}$ (see also $(7.2 .6),(7.3 .2)$ and $(7.2 .6)$ ).

Let $R=\tau_{4, \Sigma} / \tau_{3, \Sigma}$ be the iactor-sheaf of the embeding $j$. Hence, we obtain the exact sequence: (c) $0 \longrightarrow \tau_{3, \Sigma} \longrightarrow \frac{j}{\longrightarrow} \tau_{4, \Sigma} \stackrel{\beta}{\longrightarrow} \longrightarrow 0$ of sheaves on the family $\ell^{3}$.

(8.1.8) From the cited corollaries and proposition we derive also that the embedding. j: $\tau_{3, \Sigma} \subset \tau_{4, \Sigma}$ corresponds to the embeddings $\quad \mathrm{H}_{\mathrm{C}} \cap \mathrm{H}_{\overline{\mathrm{C}}} \longrightarrow \mathrm{H}_{\mathrm{C}}$ of subspaces of $\sum\left(\mathrm{V}_{2}\right)$; remember that $\mathrm{P}\left(\mathrm{H}_{\mathrm{C}}\right)=\operatorname{Span}(\mathrm{C}) C$ $C P\left(\sum\left(\mathrm{V}_{2}\right)\right), P\left(\mathrm{H}_{\overline{\mathrm{C}}}\right)=\operatorname{Span}(\overline{\mathrm{C}}) \subset \mathrm{P}\left(\sum\left(\mathrm{V}_{2}\right)\right)$, and $c+\bar{c}=c_{1}=\sigma_{2,0}(I) \cdot x_{10}<\operatorname{span}\left(c_{1}\right)=$ $=P\left(\sum\left(V_{2}\right)\right)$, where $\left.I=P\left(V_{2}\right)\right)$. ( ibid. $)$.

But the last means exactly that the epimorphism $\beta: \tau_{4, \Sigma} \longrightarrow R=\tau_{4, \Sigma} / \tau_{3, \Sigma}$ coincides with the dual of the map

$$
\begin{aligned}
\mathrm{H}^{0}\left(\varphi_{\mathrm{C}}\right): \mathrm{H}^{0}\left(\mathrm{C}, \mathrm{O}_{\mathrm{C}}\right) & \longrightarrow \mathrm{H}^{0}\left(\mathrm{C}, \mathrm{O}(1) \otimes \mathrm{O}_{\mathrm{C}}\right) \simeq \\
& \simeq \mathrm{H}^{0}\left(\mathrm{C}, \mathrm{O}_{\mathrm{P}\left(\sum\left(\mathrm{V}_{2}\right)\right)}(1) \otimes \mathrm{O}_{\mathrm{C}}\right)
\end{aligned}
$$

(see the sequence (1") and tine description of the map $\varphi_{C}$ in the beginning of the Remark). In fact, the description of the epimorpinism $\beta: \tau_{4, \Sigma} \longrightarrow R$ corresponds to the description of the global sections of 
the linear system $\left|\mathrm{P}\left(\mathrm{H}_{\mathrm{C}} \cap \mathrm{H}_{\overline{\mathrm{C}}}\right)\right|_{\mathrm{C}}|=| \mathrm{C} \cap \overline{\mathrm{C}} \mid$ on $\mathrm{C}$, defined by the map $\varphi_{C}$. Therefore, the exact sequence (1") takes the form:

$$
\begin{aligned}
& \text { (1'i) } 0 \longrightarrow\left(\tau_{4, \Sigma} / \tau_{3, \Sigma}\right)^{*}(\mathrm{C}) \stackrel{\mathrm{H}^{\circ}\left(\varphi_{\mathrm{C}}\right)=\beta^{*}}{\longrightarrow} \tau_{4, \Sigma} \text { (c)- } \\
& \longrightarrow \mathbb{P}_{e^{3}}(\mathrm{c}) \longrightarrow 0 \text {, }
\end{aligned}
$$

therefore

$$
\mathbb{R}_{e^{3}(c)} \simeq \tau_{3, \Sigma^{*}}(c) \text {. }
$$

So, we formulate the following

(8.1.9) PROPOSITION ( The open Tangent Bundle Theorem for the family $l^{3}$ of rational normal cubic curves on $x$ ). Let $U=\left\{c \in \ell^{3}: c\right.$ and $\bar{c}$ are smooth rational normal cubic curves on $X=x_{10}=G \cap P\left(V_{8}\right) \cap Q$, such that $N_{C / X}$ and $\mathbb{N}_{\bar{C} / X}$ are isomorphic to $0_{P^{1}} \oplus 0_{P^{1}}(1)$ ( see $\S 5.1$ ), and also, such that the surfaces $S_{Z}$ and $S_{C}$ are rational normal cubic scrolls ( see (2.2.4) (1) and $\S 5.2$ ) and $\left.N_{S_{1} / W} \otimes O_{C} \simeq N_{S_{1} / W} \otimes o_{\bar{C}} \simeq 0_{P^{1}}(2) \oplus 0_{P^{1}}(2)\right\} \subset l^{3}$. Then, there exists a naturally defined isomorphism

$$
\left.\left.\tau_{3, \Sigma}\right|_{0} \longrightarrow \mathbb{H}_{e^{3}}\right|_{0}
$$

To prove the Tangent Bundle Theorem (8.1.9) we need: (1). to prove Lemma $(8.1 .3)$, and (2). to globalize the local constructions, leading to the sequence ( $\left.1^{\prime \prime \prime}\right)$. 


\section{(8.1.10) Proof of Lemma (8.1.3).}

We shall suppose in addition that the surface $S_{C}=\left(U I: I-a\right.$ line in $\left.P^{4} \& I \in C\right)$ is a rational normal cubic scroll ( see (5.2.1); by the way, the surface $S_{C}$ cannot be a cone (see Corollary (5.2.3) ) ). Though this superfluous restriction will be not used in the proof the author supposes that the normal sheaves $\mathrm{N}_{C / Y}$ for the curves $C$ of the type $(5.2 .1)(2 . a)$ ( see aiso Corollary $(5.2 .3))$, where $Y=$ $=Y_{5}$ is the threefold, defined below ( see (1), and (7) ), are isomorphic to $0_{P^{1}}(1) \oplus 0_{P^{1}}(3)$. In any case, the last does not prevent us from proving $(8.1 .3)$.

(1) It is well known ( see $[F,(B .5 .8)]$ ) that the tangent bundle $T(G(2, \nabla))$ is isomorphic to the bundle of homomorphisms $\operatorname{Hom}\left(\tau_{2, \nabla}, \tau_{3, V^{*}}^{*}\right)=\tau_{2, V}^{*} \otimes \tau_{3, V^{*}}^{*}$. Let $\mathrm{P}^{3}=\mathrm{P}\left(\nabla_{4}\right)$, where $0 \subset \nabla_{4} \subset \mathrm{V}$. Then, the embedded cycle $\sigma_{1,1}\left(P^{3}\right)$ carries out, in practice, an embedding of the Grassmannian $G\left(2, \nabla_{4}\right)$ into $G(2, V)$; in particular, we have the exact sequence:

$$
\begin{aligned}
0 \rightarrow \operatorname{Hom} & \left(\tau_{2, \nabla_{4}}, \tau_{2, V_{4}^{*}}^{*}\right) \rightarrow \operatorname{Hom}\left(\tau_{2, V}, \tau_{3, V^{*}}^{*} \otimes 0 \sigma_{1,1}\left(P^{3}\right)^{-}\right. \\
& \rightarrow N\left(\sigma_{1,1}\left(P^{3}\right), G\right) \longrightarrow 0 .
\end{aligned}
$$

We can derive from here that the normal sheaf $N\left(\sigma_{1,1}\left(P^{3}\right), G\right)$ equals to the restriction

$$
\tau_{2, \mathrm{~V}}^{*} \otimes 0_{\sigma_{1,1}\left(P^{3}\right)}
$$


Let now $Y=Y_{5}=G \cap P^{6}$, and let $P^{3} \subset P^{4}$ is choosen in such a way that $\sigma_{1,1}\left(P^{3}\right)$ intersects $\mathbf{Y}$ transversely; we shall suppose also that $Y$ is choosen to be a smooth interection. The intersection $q=\sigma_{1,1}\left(P^{3}\right) \cap Y$ is a conic; we shall suppose in addition that the surface $s_{q}=\left(\bigcup_{1}: 1\right.$ - a line in $\left.p^{4} \& I \in q\right)$ is a smooth quadric ( see $\S 4.3$ (1)) . Then we shall have: $N_{q / Y}=: N(q, Y)=N\left(\sigma_{1,1}\left(p^{3}\right) \cap P^{6}, G \cap P^{6}\right)=$
$=N\left(\sigma_{1,1}\left(P^{3}\right), G\right) \otimes O_{q}=\tau_{2, V}^{*} \otimes o_{q}=o_{q}(1) \oplus o_{q}(1)$.

(2) Iet now $I$ be a line Iying on $Y=Y_{5}$. It is easy to see that $N_{1 / Y}$ is one of the sheaves $O_{1} \oplus O_{1}$ or $O_{1}(-1) \oplus O_{1}(1)$. In $\left[I_{1},(5.2)\right]$ ( see aiso $[I 4,(7.2)]$ ) was proven that $N_{1 / Y}=O_{1} \oplus 0_{1}$ for the general line $I \subset Y_{5}$. ( see also $[F N]$ ).

(3) Let now $q$ and $I$ be a conic and a line, lying on $Y=Y_{5}$ and let $q$ and $I$ intersect in a point. Let $H=P^{5} \subset P^{6}$ be a sufficiently general hyperplane through $q \cup 1$, such that the surface $S=S_{5}=Y \cap H$ is smooth. The surface $S$ is a Del Pezzo surface of degree 5 in $P^{5}=H \subset P^{6}$. The surface $S=S_{5}$ is obtained from the projective plane $F^{2}$ after blowing-up of four points $x_{1}, x_{2}, x_{3}$ and $x_{4} \in P^{2}$. As $S_{5}$ is choosen to be smooth, then no three of the points $x_{1}, x_{2}, x_{3}$ and $x_{4}$ are collinear.

(4) Iet now $z \in \mathrm{P}^{2}$ be a point, which does not lie on the union of the lines $I_{i j}=\left\langle x_{i}, x_{j}\right\rangle, 1 \leqslant i<j \leqslant 4$. 


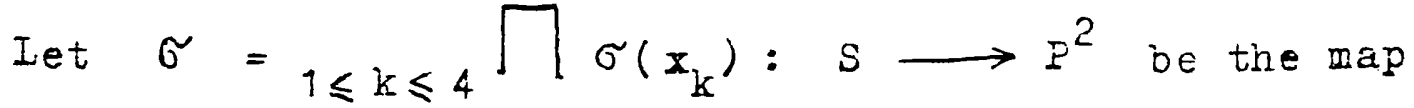
described in (3). The inverse of $\sigma$ is the rational map $\varphi$ given by the non-complete linear system

$$
\left|o_{P^{2}}\left(3-x_{1}-x_{2}-x_{3}-x_{4}\right)\right|: P^{2}-\cdots \rightarrow P^{5} \text {. }
$$

Let now $Q$ be a conic in $F^{2}$ through the points $x_{1}, x_{2}, x_{3}$ and $z$. It is easy to see that the proper $\sigma$-preimage $c \subset s=s_{5}$ of the conic $(Q=0)<P^{2}$. $Q \in H^{0}\left(O_{P^{2}}\left(2-x_{1}-x_{2}-x_{3}-z\right)\right)$ is a rational normal cubic curve.

The line 1 (see (3)) is one of the ten lines, which lie on the Del Pezzo surface $S=S_{5} \subset \mathrm{P}^{5}$. Obviously, we can suppose that the morphism $\sigma$ is choosen in such a way that $1=\sigma^{-1}\left(\mathrm{x}_{4}\right) \subset \mathrm{S}_{5}$.

Let $(t)=\left(t_{1}: t_{2}\right)$ be the homogeneous coordinates of $P^{1}=\left|0_{P^{2}}\left(2-x_{1}-x_{2}-x_{3}-z\right)\right| \quad$. Evidently, the proper preimage $C_{(t)}$ of the conic $Q_{(t)} \in \mathrm{P}^{1}=\mathrm{P}^{1}(t)$ splits iff $Q(t)$ passes through the point $x_{4}$. Let $(1)=(1: 1)$, and let $x_{4} \in Q_{(1)}$. Then $C_{(1)}=$ $=q \cup 1$, where $q$ is the conic and $I$ is the Iine $I=\sigma^{-1}\left(x_{4}\right)$; obviously, $(q, I)_{S}=1$.

(5) Let now

$$
\beta_{(t)}: Y_{(t)} \longrightarrow Y=Y_{5},(t) \neq(1) \text {, }
$$
be the blowing-up of the curve $C_{(t)}\left(\subset S_{5}\right) \subset Y=Y_{5}$; let also $S_{(t)}=\beta(t)^{-1}\left(C_{(t)}\right)$ be the ruled surface over the curve $C_{(t)}, \quad(t) \neq(1)$. It is easy to see that the normal sequence for $C_{(t)} \subset S_{5} \subset Y$ is : 


$$
0 \longrightarrow \mathrm{O}_{(t)}(1) \longrightarrow \mathrm{N}_{\mathrm{C}_{(t)} / \mathrm{Y}} \longrightarrow \mathrm{O}_{\mathrm{C}_{(t)}}(3) \longrightarrow 0 .
$$

Therefore, ${ }^{N} C_{(t)} / Y=O_{C_{(t)}}(a) \oplus O_{C}(t)(b)$, where

$$
\begin{aligned}
& a+b=4 \text { and } 1 \leqslant a \leqslant b \leqslant 3 \text {. } \\
& \text { Let } S=\overline{(t) \neq(1) \bigcup S_{(t)}} \subset \\
& \overline{(t) \neq(1) \cup Y}(t)_{\bigcup_{(t)}} \text {. }
\end{aligned}
$$

obviously, $S($ resp. $y$ ) is a bundle of rational surfaces (resp. of rational threefolds) over the projective Iine $P^{1}=P^{1}(t)$.

(6) Let the general rational normal cubic $C \subset Y_{5}$ has a normal bundle $O_{C}(1) \oplus O_{C}(3)$. Then we can choose the hyperplane $\mathrm{H}$ ( and, hence, the surface $S_{5}=Y_{5} \cap \mathrm{H}$ ) through the given degenerate rational normal cubic $c_{(1)}=q \cup 1$ ( see (3)), and the point $z \in P^{2}$ ( see (4)) in such a way that $N_{C_{(t)}} / Y$
$=O_{C}(t)$
(1) $\oplus O_{C}(t)$
(3) for any
(t) $\in P^{1}(t)$ in some neighbourhood of $(t)=(1)$. We can also suppose that the normal sheaves of $q$ and $I$ are as in (1) and (2) , i.e. $N_{q / Y}=O_{q}(1) \oplus O_{q}(1)$ and $N_{I / Y}=O_{I} \oplus O_{I}$ The exceptional surfaces $S_{(t)}=\beta_{(t)}^{-1}\left(C_{(t)}\right) \subset Y(t)$ are isomorphic to the ruled surface

$$
\mathbb{F}_{2}=P\left(N_{C(t)} / Y\right)=P(O(1) \oplus O(3)) \text {, for all }
$$
$(t) \neq(1)$, around
(1) The surface
$S(1)$

$=e \backslash[(t) \neq(1)$

U

$S(t)$

is a degeneration of $\mathbb{F}_{2}$; on the other hand, $S(1)$ is an union of the two quadrics the quadric $A$ over $q$ and the quadric $B$ over $I$. 
The smooth quadics A and B intersect along the commongenerator $A \cap B$, because $(q, I)_{S}=1$. But then the union $A \cup B$ cannot be a degeneration of the family of $\mathbb{F}_{2}$ 's described above. Consequertiy, the general rational normal cubic curve $C \subset Y=Y_{5}$ has a normal bundle $N_{C / Y}=o_{C}(2) \oplus 0_{C}(2) .(\operatorname{see}(5))$.

(7) Let now $C \subset X=X_{10}$ be a general rational normal cubic curve. In particular, $C$ is embedded in the rational normal cubic scroll $S_{I} \subset W$ as a hyperplane section (see Prop. $(2.2 .4)(1)$ and Cor. (5.3.2)).

Let $H=P^{6} \subset P^{7}$ be a sufficiently general hyperplane through the curve $C$, such that the intersection $Y=Y_{5}=W \cap H$ is smooth. In particular, let $\mathrm{H}$ intersects $S_{1}$ transversely along the curve $C$. Then $N_{S_{I}} / W \otimes O_{C}=N_{\left(S_{I} \cap H\right) /(W \cap H)} \otimes O_{C}=N_{C / Y}$. Clearly, we can also suppose that the curve C is sufficiently general in $Y=Y_{5}$, i.e. $\mathrm{N}_{\mathrm{C} / \mathrm{Y}}=$ $=O_{C}(2) \oplus O_{C}(2)$. But then $N_{S_{I}} / W \otimes O_{C}=N_{C / Y}=$ $=o_{C}(2) \oplus O_{C}(2)$. Lemma $(8.1 .3)$ is proved. 
8.2. Proof of the Tangent Bundle Theorem (Prop. (8.1.9)). (8.2.1) The proof of the T.B.T. consists in the repeating of the local constructions from the beginning globally over the whole family $\epsilon^{3}=\zeta^{3}(x)$. For this purpose we introcuce the following subvarieties of the projectivized bunale $P_{C^{3}}(\Sigma)$ on the places of tine curve $C \subset \mathbb{X}=$ $=\mathrm{X}_{10}$ and of the surface $\mathrm{S}_{1} \subset \mathrm{W}$ :

$$
\begin{aligned}
& D_{e^{3}}=\left\{(C, x) \in C^{3} \times x: x \in C\right\} \\
& D_{e^{3}}(G)=\left\{(C, x) \in l^{3} \times w: x \in S_{1}\right\} ;
\end{aligned}
$$

remind that $C+\bar{C}=C_{1}=S_{1} \cdot Q \subset S_{1} \subset P\left(\Sigma\left(\nabla_{2}\right)\right)$ where $I=P\left(V_{2}\right)$.

(8.2.2) The pairs of successive embedaings

$$
D_{b^{3}} \subset e^{3} \times x \subset l^{3} \times w \quad \text { and }
$$

$$
D_{G^{3}} \subset D_{G^{3}}(G) \subset C^{3} \times w
$$

globalize respectively the pairs of embedings $C<\mathrm{X} \subset \mathrm{W}$ and $C \subset S_{1} \subset W$. Correspondingly to the normal sequences $(8.1 .1)(1)$ and $(8.1 .1)$ (2) they induce the sequences of normal sheaves :

$$
\begin{aligned}
& (1, l) \quad 0 \rightarrow N\left(D l_{l^{3}}, l^{3} \times x\right) \longrightarrow N\left(D_{l^{3}}, l^{3} \times w\right) \longrightarrow \\
& \longrightarrow \mathbb{N}\left(l^{3} \times \mathrm{X}, \zeta^{3} \times w\right) \otimes \mathrm{O}_{\mathrm{C}^{3}} \longrightarrow 0 \text { and } \\
& (2, \zeta) \quad 0 \rightarrow N\left(D_{G^{3}}, D_{G^{3}}(G)\right) \rightarrow N\left(D_{b^{3}}, \ell^{3} \times w\right) \longrightarrow
\end{aligned}
$$$$
\longrightarrow \mathbb{N}\left(D_{l^{3}}(G), C^{3} \times W\right) \otimes O_{D_{l^{3}}} \longrightarrow 0 \text {. }
$$

(8.2.3) Let $O(1)$ be the antitauthological sheaf on the projective bunãle $P_{G(2, \nabla)}(\Sigma) \longrightarrow G=G(2, V)$. 
According to the definition of the bundle $\sum$ (see (6.3.3)), the sheaf $\sum$ on $G$ is embedied in a natural way in the shear $V_{8} \otimes O_{G}$ (corresponding to the constant bundie $G \times V_{8}$ over $\left.G=G(2, V)\right)$. In fact, for every $v_{2} \in G=G(2, V)$, the embeding of the corresponding fibers $\sum\left(\mathrm{V}_{2}\right) \subset\left(\mathrm{V}_{8} \otimes \mathrm{O}_{\mathrm{G}}\right)\left(\mathrm{V}_{2}\right) \quad$ coincides with the natural embeading $\quad\left(\nabla_{2} \wedge \mathrm{V}\right) \cap \nabla_{8} \subset \nabla_{8}$. Thetefore, the sheaf $O$ (1) coincides with the restriction of the sheaf $O_{P\left(\nabla_{8}\right)}(1)=O_{P\left(\Lambda^{2} \nabla\right)} \otimes O_{P\left(\nabla_{8}\right)}$ on the subvariety $P_{G}\left(\sum\right) \subset P_{G}\left(\nabla_{8} \otimes O_{G}\right)=G \times P\left(\nabla_{8}\right),($ the nold" o(1), see above ).

(8.2.4) As in the local case we multiply the sequence $(2, \zeta)$ by the sheaf $O(-1)$ :

$$
\left(2^{\prime}, G\right) \quad 0 \rightarrow N\left(D_{C^{3}}(G)\right) \otimes O(-1) \longrightarrow
$$

$\longrightarrow N\left(D_{C^{3}}, l^{3} \times w\right) \otimes O(-1) \rightarrow N\left(D_{e^{3}}(G), C^{3} \times w\right) \otimes O_{b^{3}} \otimes O(-1) \longrightarrow 0$ Similarly, we obtain the sequence $(1, l) \otimes O(-1):$

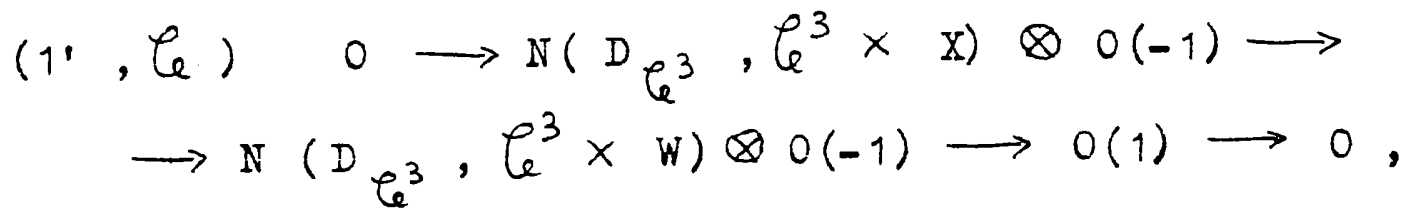
since $\mathbb{N}\left(e^{3} \times \mathrm{x}, e_{e}^{3} \times w\right)=0(2)$.

(8.2.5) Let, for the brevity, cenote by $B$ the sheaf $\mathbb{N}\left(D_{C^{3}}, 6^{3} \times w\right) \otimes O(-1)$ and let $\alpha$ and $\beta$ are the natural projections for the Fano femily $D C^{3}$ : 


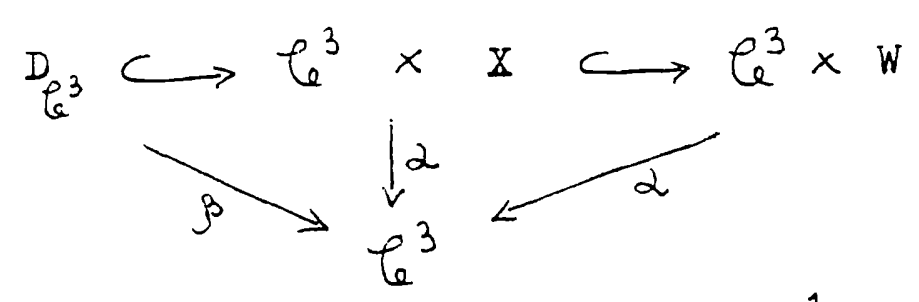

As before, using the equality $R_{\beta}^{1} N\left(D l^{3}, l^{3} \times x\right)=0$ which can be obtained on the level of fibers from the local equality $\mathrm{H}^{1}\left(\mathrm{C}, \mathrm{N}_{\mathrm{C} / \mathrm{X}}\right)=0$, and also the relative duality of Serre, we obtain :

$$
\Theta_{l^{3}}=R_{\beta}^{1}\left(N\left(D_{l^{3}}, \zeta^{3} \times x\right) \otimes O(-1)\right)
$$

(here $R^{i}$. is the operation of taking the $i^{\text {th }}$ direct image ).

(8.2.6) In a similar way, using the local equalities:

$$
\mathrm{H}^{i}\left(\mathrm{~N}_{\mathrm{S}_{1} / \mathrm{W}} \otimes \mathrm{O}_{\mathrm{C}} \otimes \mathrm{O}(-1)\right)=0, \quad i=1,2, \ldots
$$

we derive the identities :

$$
\begin{aligned}
& (5, G) R_{\beta}^{1}\left(N\left(D_{l^{3}}(G), \ell^{3} \times W\right)\right) \otimes O_{D_{l^{3}}} \otimes O(-1)=0, \\
& i=1,2, \ldots
\end{aligned}
$$

(8.2.7) As is already clear, $N\left(\ell^{3} \times \mathbb{Z}, \ell^{3} \times w\right)=0(2)$. Using the obtained just above and taking the direct $\beta$-images of the sequences $\left(1^{\prime}, C\right)$ and $\left(2^{\prime}, l\right)$, we obtain :

$$
\begin{aligned}
\left(2^{\prime \prime}, C_{e}\right) \quad 0 & \rightarrow \beta_{*}\left(N\left(D_{l^{3}}, D_{l^{3}}(G)\right) \otimes O(-1)\right) \longrightarrow \beta_{*} B \longrightarrow C \\
0 & \longrightarrow R_{\beta}^{1}\left(N\left(D_{l^{3}}, D e^{3}(G)\right) \otimes O(-1)\right) \longrightarrow R_{\beta}^{1} B \rightarrow 0
\end{aligned}
$$

and :

$$
(1 ", l) \quad 0 \longrightarrow \beta_{*}\left(N\left(D l_{l^{3}}, \zeta^{3} \times x\right) \otimes 0(-1)\right) \longrightarrow \beta_{*} B \longrightarrow
$$$$
\longrightarrow \beta_{*} O(1) \longrightarrow \mathscr{U}_{e^{3}} \longrightarrow R_{\beta}^{1} B \longrightarrow R_{\beta}^{1} O(1) \longrightarrow 0 \text {, }
$$

where $\beta_{*}=R_{\beta}^{0}$. 
(8.2.8) Now, we introduce tine variety $D_{e^{3}}(\langle\cdot\rangle)=\left\{(c, x): c \in \ell^{3}, x \in\langle c\rangle=\operatorname{span}(c)\right\}$. As $\operatorname{Span}(C) \subset P\left(\sum(C)\right)=P\left(\sum\left(V_{2}\right)\right)$, then

$D_{e^{3}}(\langle\cdot\rangle)$ is embedded naturally in the projectivized bundle $\mathrm{P}_{e^{3}}\left(\sum\right)$. In fact, the bundle $\sum$ is defined, originally, on the Grassmannian $G=G(2, V)$, but it is defined also on the subsets of $G(2, V)$ and on the preimages of these suosets under morphisms; as usual, we drop the symbols of the maps. From the Remark $(6)$ (see $(8.1 .5)),(8.1 .7)$ and $(8.1 .8)$, we conclude that

$$
D_{\ell^{3}}(\langle\cdot\rangle)=P_{\ell^{3}}\left(\tau_{4, \Sigma}\right),
$$

( see also the Corollary $(7.3 .3)$ ).

The Fano family $D_{e_{e}^{3}}$ is embedded identically in the projectivizated bundle $D_{e^{3}}(\langle\cdot\rangle)=P_{e^{3}}\left(\tau_{4, \Sigma}\right)$; in particular, the projection $\beta: D_{\varphi^{3}} \longrightarrow C^{3}$ is a restriction of the projection $\alpha: D \ell_{l^{3}}(\langle\cdot\rangle)=$ $=P_{\epsilon^{3}}\left(\tau_{4, \Sigma}\right) \longrightarrow \zeta^{3}$. From the properties of the antitauthological sheaf $O(1)$ on $P_{e^{3}}\left(\tau_{4, \Sigma}\right)$ we have:

$$
\beta_{*} O(1)=\tau_{4, \Sigma}^{*} \text { and } R_{\beta}^{1} O(1)=0 \text {. }
$$

(8.2.9) Let we denote the sheaf $N\left(D_{C_{e}^{3}}, D_{l_{e}^{3}}(G)\right) \otimes O(-1)$ by $A$. Taking into account the identity

$$
\beta_{*}\left(N\left(D \epsilon_{e^{3}}, \epsilon^{3} \times x\right) \otimes 0(-1)\right)=0 \text {, }
$$

which follows from the corresponding local equality, we conclude, just as in the local case, that the exact sequence 
(1", $\left.l_{e}\right)$ can be written in the form:

$\left(1^{\prime \prime \prime}, \ell^{\prime}\right) \circ \longrightarrow \beta_{*} A \longrightarrow \tau_{4, \Sigma}^{*} \longrightarrow \mathscr{H}_{\ell^{3}} \longrightarrow \mathrm{R}_{\beta}^{1} \Delta \longrightarrow 0$, (see the"local"sequences $(8.1 .5)(1 ")$ and $(8.1 .8)$ (1"1)) . According to the comments in the Remark (8.1.6) (6), the sheaf $\beta_{*} A$ coincides with the factorsheaf $R=$ $=\left(\tau_{4, \Sigma} / \tau_{3, \Sigma}\right)^{*}$. If, moreover, the curve $C$ ( $c \in C^{3}$ ) is general ( in the sense of the conditions of Proposition (8.1.9)), then the first direct image $R_{\beta}^{1} A$ vanishes over $C$, since the fiber of $R_{\beta}^{1} A$ over $c \in 0<e^{3}$ is $\mathrm{H}^{1}\left(\mathrm{~N}_{\mathrm{C} / \mathrm{S}_{1}} \otimes \mathrm{O}(-1)\right)=\mathrm{H}^{1}\left(\mathrm{C}, \mathrm{O}_{\mathrm{C}}\right)=0$ $\left(C \simeq P^{1}\right)$. The open T.B.T. (Proposition $(8.1 .9)$ ) is provea (8.2.10) REVARK.

The correct formulation of the T.B.T. must take into account a joint geometrical interpretation of the sequences $\left(1, l_{e}\right)$ and $\left(2, l_{l}\right)$, taking into consideration also the disposition of the locus $\zeta_{\text {ex }}^{3} \subset \ell^{3}$, where the local constructions fail; it is already clear that $\ell_{e x}^{3} \subseteq \ell^{3} \backslash \sigma$ $(\operatorname{see}(8.1 .4)(5)$ and $(8.1 .3))$.

$(8.2 .11)$ The geometry of the Fano threefold $\mathrm{X}=\mathrm{X}_{10}$ can provide an additional useful information by means of the geometrical analogue of the just proven open T.B.T. First, the exact sequence ( 1 "', $\zeta$ ) induces a natural isomorphism $\psi:\left.\tau_{3, \Sigma}^{*}\right|_{U} \longrightarrow \Psi_{\left.e^{3}\right|_{U}}$ over the open subset $u \subset e^{3}$. The definition of $\mathrm{H}^{0}\left(\varphi_{\mathrm{C}}\right)$ in (8.1.5) (1.") shows that the fibre $\tau_{3, \Sigma}$ (C) coincides with the 
embedded piane $\mathbb{H}_{\mathrm{C}} \cap \mathrm{H}_{\bar{C}} \subset \sum(\mathrm{C})\left(=\sum\left(\mathrm{V}_{2}\right)=\right.$ $\left.=\sum(\overline{\mathrm{C}})\right)$; see also the Corollary $(7.2 .6)$ (2). inere, as Usual, $\mathrm{C}+\mathrm{C}=\mathrm{C}_{\mathrm{P}\left(\mathrm{V}_{2}\right)}, \mathrm{P}\left(\mathrm{H}_{\mathrm{C}}\right)=\langle\mathrm{C}\rangle, \mathrm{P}\left(\mathrm{H}_{\overline{\mathrm{C}}}\right)=\langle\overline{\mathrm{C}}\rangle$ The dual map $\Psi^{*}$ defires the isomorphism :

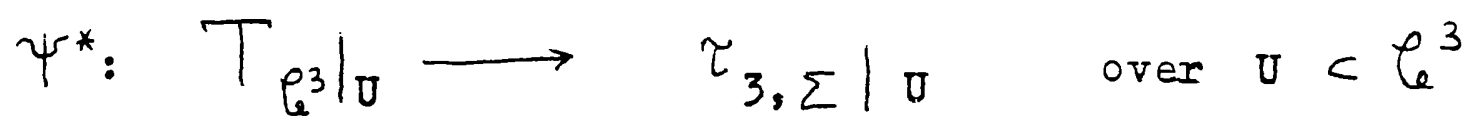

(nere $T_{\ell^{3}}=\mathscr{H}_{\ell^{3}}^{*}$ is the tangent sheaf of the famiIy $\left.e^{3}\right)$.

So, we can formulate the following :

(8.2.12) THEOREM (Geometric (open) T.B.T. for $\ell^{3}$ ).

There is a naturally defined isomorphism (see (8.1.9)) :

$$
\psi^{*}:\left.T_{\left.e^{3}\right|_{0}} \longrightarrow \tau_{3, \Sigma}\right|_{0} \text {, }
$$

such that $\Psi^{*}$ coincides with the natural map

$$
\psi^{*}(\mathrm{C}): T_{e^{3}}(\mathrm{C}) \stackrel{\sim}{\longrightarrow} \mathrm{H}_{\mathrm{C}} \cap \mathrm{H}_{\overline{\mathrm{C}}} \subset \sum\left(\mathrm{V}_{2}\right) \subset \mathrm{V}_{8}
$$

on the fiber $T_{e^{3}}(c) ;($ see Corollary (5.3.2) and Corollary (7.2.6) for the definitions of $\mathrm{V}_{2}, \overline{\mathrm{C}}, \mathrm{H}_{\mathrm{C}}$ and $\left.\mathrm{H}_{\overline{\mathrm{C}}}\right)$.

$\S$ 9. Tangent Bundle Theorem for the family of conics on the Fano threefold $X=x_{10}$.

9.1. In the present section we shall follow some results of the Thesig of $D$. Logachev $\left[\mathrm{I}_{1}\right]$ ( see also [I2] and $[\mathrm{P}]$ ) on the family of conics on $X=X_{10}=G \cap P\left(V_{8}\right) \cap Q$. The original idea of representing the family of conics on $\mathrm{X}_{10}$ as a degeneration Iocus, resp. as a zero scheme of a section of sone vector bunde, and the corresponding statement of the tangent bundle theorem, belongs to Iogachev. 
The mair observations in the present work are : (1). The use of the simpie fact that the conics and the ratiorai nomal cubics on $x_{10}$ correspond to the standard splittings of the codim. 2 -cycles on $x_{10}$ of the types $\sigma_{1,1} \cap x_{10}$ and $\sigma_{2,0} \cap x_{10}$ respectively; the last generate the subring of the ring of cycles of codimension 2 on $X=X_{10}$, which are induced from cycies on the Grassmannian $G(2, V)$;

(2). The possibility to perform the constructions of Logacher also to the family of the rational normal cubic curves on $\mathrm{x}_{10}$. The two marked families are, probably, the only families of curves on $\mathrm{x}_{10}$, for which the mentioned methods and representations are adequate.

In particular, (1), together with the geometrical interpretations of the tangent bundle theorems for both families

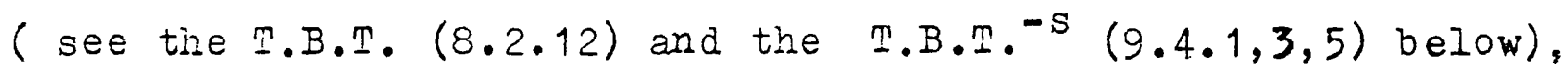
grounds us to carry out a geometrical approach to the Abel-Jacobi maps for some families of curves, naturally arising on $\mathbb{Z}($ see for ex. [CG]). Let, for example:

$$
\ell_{1}^{5}=\left\{c \text { - a curve : c c x, } p_{a}(c)=1, \operatorname{aeg}(c)=5\right\}
$$

be the family of the linearly nomal elliptic quintics on $\mathbf{X}$; the last corresponds to the set of components of the aegenerate canonical curves of degree 10 on $\mathrm{x}_{10}$ into pairs of eliiptic curves. The T.B.T. ${ }^{-S}$ for the families $F=$ $=\left\{\right.$ conics on $\left.x_{10}\right\}$ and $\ell^{3}=\left\{\right.$ rat.norm. cubics on $\left.x_{10}\right\}$, enables us to perform a geometrical approach to the Abel-Jacobi map of the family $W=l^{3}+F=$ 


$$
\begin{aligned}
& =\left\{c+q: c \in l^{3}, q \in F\right\} . \\
& \quad \text { Let } l_{e}=l_{1}^{5} \cap W=\left\{c+q: c \in l^{3}, q \in F,(c, q)=2\right\}
\end{aligned}
$$

be the threedimensional family, the elements of which are, by definition, degenerate eiliptic quintics on $x$, which split into a sum of a rat.nom. cubic and a conic, intersecting between them in two points. The results in the paper [V] of Claire Voisin on the Quartic Double Solids give us a hope to suppose that the general points $z$ of the two-dimensional subvariety $z$ of the Intermediate Jacobian $J\left(x_{10}\right)$ of $x_{10}$, which is the Abel-Jacobl image of the "diagonal" family $\ell_{e}$, are double points of the theta- divisor (4) $\left(x_{10}\right) \subset J\left(x_{10}\right)$. The author's suggest is that the tangent cones $\mathbb{T C}_{\mathbb{Z}}(\Theta)$ to the theta divisor $(4)$ at the singular points $z$ of $H$, are quadrics of rank $=6$. The last will provide us with a geometrical proof of the non-rationality of the Fano threefold $\mathrm{X}=$ $=X_{10}=G(2,5) \cap P^{7} \cap Q(\sec [V, \S 4])$.

9.2. In (6.2.1) was defined the bundle $\mathrm{Pf} \longrightarrow P\left(\nabla^{*}\right)$; the fiber $\operatorname{Pf}\left(\mathrm{V}_{4}\right)$ over the element $\nabla_{4} \in P\left(V^{*}\right)$ was defined to be the Plücker quadric, corresponding to the 4-space $\mathrm{V}_{4}$ ( for more details, see $\S 6.2$ ). It was proven that the corresponding invertible sheaf $P f$ over $P\left(V^{*}\right)$ is isomorphic to the sheaf (det $\left.\tau_{4, \nabla}\right)^{-1}=O_{P\left(V^{*}\right)}(1)$.

Iet now $V_{8}, V_{8} \subset V_{10}=\Lambda^{2} \mathrm{~V}$ be choosen sufficiently general, such that: 
(i) the variety $X=G \cap P\left(V_{8}\right) \cap Q$ is smooth ;

(ii) aim $\left(v_{8} \cap\left(\wedge^{2} v_{4}\right)\right)=4$, for every $v_{4} \in F\left(V^{*}\right)=$ $=G(4, V)$.

Then we can define correctly a bundle $M$ over $P(V)$ with 4-aimensional fibers $M\left(V_{4}\right)$. such that

$$
M\left(\nabla_{4}\right)=\Lambda^{2} \nabla_{4} \cap V_{8}, \nabla_{4} \in P\left(V^{*}\right)=G(4, \nabla) .
$$

On the other hand, the restriction of the Plücker bundle $P f \longrightarrow P\left(V^{*}\right)$ (regarded as a bundle of quadrics on $\Lambda^{2} \tau_{4, V} \longrightarrow P\left(\nabla^{*}\right)$ ) to the subbundie $M=\Lambda^{2} \tau_{4, V} \cap \nabla_{8} \subset \Lambda^{2} \tau_{4, \nabla}$, represents the bundle $P f$ as a subbundle of $S^{2} M^{*} \longrightarrow P\left(V^{*}\right)$; moreover Pf has fibers

$$
\operatorname{Pf}\left(\nabla_{4}\right)=H^{\circ}\left(P\left(\Lambda^{2} \nabla_{4} \cap \nabla_{8}\right), O\left(2-\left(\sigma_{1,1}\left(P\left(V_{4}\right)\right) \cap P\left(V_{8}\right)\right)\right)\right.
$$

(see Corollary $(6.2 .3))$.

We can also define an analogue to the bundle $Q \rightarrow G(2, V)$ which was regarded as a subbundle of $s^{2} \Sigma^{*} \longrightarrow G(2, V)$, namely :

Let $X=G \cap P\left(\nabla_{8}\right) \cap Q$, where $Q$ is a quadric. We define the bundle (the invertible sheaf) $Q$ over $P(V)$ such that :

(1) $Q$ is a subbundle of $s^{2}\left(\Lambda^{2} \tau_{4, V}\right)^{*}$;

(2) $Q\left(\mathrm{~V}_{4}\right)=\left.\mathbb{C} \cdot \mathrm{Q}\right|_{\mathrm{P}\left(\wedge^{2} \mathrm{~V}_{4}\right)} \subset \mathrm{H}^{\mathrm{O}}\left(\mathrm{P}\left(\wedge^{2} \mathrm{~V}_{4}\right), \mathrm{O}(2)\right)$, ¿.e. $\because\left(\mathrm{V}_{4}\right)$ is the restriction of the equation of the quadric $Q$, etc. 
From the conditions (i) and (ii) we derive that we can look at the bundle $Q \longrightarrow P\left(V^{*}\right)$ also as a bundle of quadrics ( a "quaciric") on the bundle $M$. Moreover, it is evident that the corresponaing sheaf $Q$ is isomorphic to the structure sheaf $0_{P\left(V^{*}\right)}$. Just as in $\S 6.3$

( see Prop. (6.3.2) ) we can prove the following :

(9.2.1) PROFOSITION (IOgachev, [I1]) .

Let $\quad \mathrm{Z} \subset \mathrm{P}_{\mathrm{P}\left(\mathrm{V}^{*}\right)}\left(S^{2}\left(\Lambda^{2} \tau_{4, \mathrm{~V}}\right)^{*}\right)$ be the set : $Z=P\left\{\left(V_{4}, q\right): V_{4} \in P\left(V^{*}\right), q \in H^{0}\left(P\left(\Lambda^{2} V_{4}\right), O\left(2-Q \cap \sigma_{1,1}\left(P\left(V_{4}\right)\right)\right.\right.\right.$ Then $Z$ is naturally isomorphic to the projectivized bunale $\quad P_{P\left(\nabla^{*}\right)}\left(0_{P\left(\nabla^{*}\right)}(1) \oplus 0_{P\left(\nabla^{*}\right)}\right)$.

Poreover, from (i) and (ii) (see above), follows, tha $Z=P\left\{\left(V_{4}, q\right): \nabla_{4} \in P\left(V^{*}\right), Q \in H^{0}\left(P\left(\wedge^{2} V_{4} \cap V_{8}\right), O\left(2-\sigma_{1,1}\left(I\left(V_{4}\right)\right) \cap P\left(V_{8}\right) \cap G\right.\right.\right.$ $=P\left\{\left(\nabla_{4}, q\right): \nabla_{4} \in P\left(\nabla^{*}\right), q \in H^{\circ}\left(P\left(M\left(\nabla_{4}\right)\right), O\left(2-\sigma_{1,1}\left(P\left(\nabla_{4}\right)\right) \cap \mathbb{X}\right)\right)\right\} \subset$ $C P_{P\left(V^{*}\right)}\left(S^{2} M^{*}\right) \quad($ see Proposition $(6.3 .2 \gamma)$. (9.2.2) From the definition of the variety $z$ follows that the fiber $\eta^{-1}\left(\mathrm{~V}_{4}\right)=\mathrm{Z}\left(\mathrm{V}_{4}\right)$ of the projective bundle $\quad \eta: Z \longrightarrow P\left(\nabla^{*}\right)$ is isomorphic to the projective Iine $\left\langle\operatorname{Pf}\left(\mathrm{V}_{4}\right), Q\left(\mathrm{~V}_{4}\right)\right\rangle=\operatorname{Span}\left\{\operatorname{Pf}\left(\mathrm{V}_{4}\right) \cup Q\left(\mathrm{~V}_{4}\right)\right\}$ in the projective space $P\left(S^{2} M^{*}\left(V_{4}\right)\right)$ of quadrics in $P\left(M\left(V_{4}\right)\right)$. 
The surface $F_{c}$ of the geometrical conics on $x=x_{10}$, the surface $F$ ( $0 f^{\prime}$ "conics on $X "$ ) and the factorsurface $F_{0}=F / i=\left\{\left(q, \bar{q} ; \nabla_{4}\right): q \in F_{c},\left(\bar{q}, \nabla_{4}\right)=i\left(q, \nabla_{4}\right)\right\}$ were defined in $\hat{\S} 4.4$ and in $\hat{\S} 4.5$ (here $i$ is the involution, wirich is correctly defined on $\vec{F}$ ). The non-correctness of the defining the rational map $(-)^{-1}: F_{c} \longrightarrow F_{c}$ arises from the fact that the Iinear span of the set $s_{q_{0}}=\left(\bigcup_{I}: I \subset P(v)-a\right.$ line, $I \in q_{c}$ where $q_{0}$ is the unique $\rho$-conic on $x$, is contained in an one-dimensional family of $\mathrm{P}^{3} \mathrm{is}^{\mathrm{s}}$; the last coincides with the line $P\left((V / U)^{*}\right)=$ $=\left\{\mathrm{P}^{3}: \mathrm{P}^{3}<\mathrm{P}^{4}=\mathrm{P}(\mathrm{V})\right.$ \& (the plane) $\left.\mathrm{P}(\mathrm{U})=\mathrm{S}_{\mathrm{q}_{0}} \subset \mathrm{P}^{3}\right\}$ $($ see $\S 4.2$ and $\S 4.3)$.

(9.2.3) Let now $\nabla_{4} \in P\left(V^{*}\right)$ and let $W$ denotes, as usual, the intersection $W=G \cap P\left(\nabla_{8}\right) \subset P\left(\nabla_{8}\right) \subset P\left(\wedge^{2} \nabla\right.$. As we know (see Proposition (2.2.3) (2)) the cycle $\mathrm{Q}\left(\mathrm{P}\left(\mathrm{V}_{4}\right)\right)=\sigma_{1,1}\left(\mathrm{P}\left(\mathrm{V}_{4}\right)\right) \cap \mathrm{P}\left(\mathrm{V}_{8}\right) \subset \mathrm{W}$ is a two-dimensional quadric. It follows from the definition of the bundle $M \longrightarrow P\left(V^{*}\right)$ ( see the introduction of $\S 9.2$ ) that $\left\langle Q\left(P\left(V_{4}\right)\right)\right\rangle=\operatorname{Span}\left(Q\left(P\left(V_{4}\right)\right)\right)=P\left(M\left(V_{4}\right)\right)$. By definition, $W \cap Q=X$. Hence, the elliptic quartic $C\left(P\left(V_{4}\right)\right.$ : ( see Corollary (3.3.3)), which is, by definition, an intersection of the cycle: $\sigma_{1,1}\left(P\left(\nabla_{4}\right)\right)$ with $X$, coincides with the intersection of tine quadrics $Q\left(P\left(V_{4}\right)\right)$ and $Q$ 
in the space $P\left(N_{i}\left(V_{4}\right)\right)=P\left(\Lambda^{2} V_{4} \cap V_{8}\right)$.

The quadric $Q\left(P\left(V_{4}\right)\right)$ defines a section of the Ffaff bundle $P f \longrightarrow P\left(V^{*}\right)$; the restrictions of the quaciric $Q$ on the fibers of the bundle $M \longrightarrow P\left(T^{*}\right)$ define a section of the bundle $Q \rightarrow P(V)$, defined above. Therefore, the condition of splitting of the curve $\mathrm{C}\left(\mathrm{P}\left(\mathrm{V}_{4}\right)\right)$ into a pair of involutive conics is equivalent to the existence of a quadric of rank $\leqslant 2$ on the line $\left\langle P f\left(\nabla_{4}\right) \cup Q\left(V_{4}\right)\right\rangle$ in the space of quadrics in $P\left(M\left(\nabla_{4}\right)\right)$. The last arguments are correct on the subset $F_{C} \backslash\left\{q_{C}\right\} \subset F_{c}\left(q_{0}\right.$ is the unique $\rho$-conic on $x)$, but it is not hard to see that the "exceptional" quadrics of $F$, which lie on the exceptional line $r_{F}^{-1}\left(q_{0}\right)($ see $(4.5 .1))$ correspond to degenerate quadrics in the bundle $P f \oplus Q$ ( see for ex. $\S 4.3$, $\S 4.4$, Claim $(4,2.1)$ and $(4.5 .1))$. The involution $i$ on $F$ identifies the elements of the exceptional curve $r^{-1}\left(q_{0}\right)$ with the elements of the curve of the $\sigma$-conics on $X .($ ibid.).

(9.2.4) The tauthological sequence :

$0 \longrightarrow 0_{\eta}(-1) \longrightarrow \eta^{*}\left(0_{P\left(V^{*}\right)}(1) \oplus 0_{P\left(V^{*}\right)}\right) \rightarrow 0_{Z}(1) \rightarrow 1$

on the projective bundle

$$
\eta: Z=P\left(O_{P\left(V^{*}\right)}(1) \oplus O_{P(V)}\right) \longrightarrow P\left(V^{*}\right)
$$

defines, just as in (6.4.4) (see also (5.4.2)), a net of quadrics on $Z$. It follows from the properties of the tatirological sequence that the projectivization of the embeading 
$0 \longrightarrow 0_{\eta}(-1) \stackrel{j}{\longrightarrow} \eta^{*}\left(O_{F\left(V^{*}\right)}(1) \oplus O_{F\left(V^{*}\right)}\right)$ represents the "embedded" points of the fibers of the Erojective bunile $Z$; these points are interpreted also as quadrics in the corresponaing spaces $P\left(M\left(V_{4}\right)\right)$. Becaise of that, as in $\S 6$, Froposition (6.4.4), we can conclude the following :

(9.2.5) CIAIr ( Iogachev, $\left[I_{1}\right]$ ) . The factorfamily (of pairs of involutive conics) $F_{0}=F / i$ is embedied naturally in the projective bunde.

$$
Z=P_{P\left(V^{*}\right)}\left(O_{P\left(V^{*}\right)}(1) \oplus O_{P\left(V^{*}\right)}\right)
$$

as a second determinantal of the net of quadrics :

$$
\begin{aligned}
& 0 \longrightarrow 0_{\eta}(-1) \stackrel{j}{\longrightarrow} \eta^{*}\left(O_{P\left(V^{*}\right)}(1) \oplus 0_{P\left(V^{*}\right)} \stackrel{i}{\longrightarrow} \eta^{*}\left(S^{2} M^{*}\right)\right. \\
& \text { i.e. } \quad F_{0}=D_{2}(\hat{\varphi}) \text {, where } \hat{\varphi}=i \cdot j .
\end{aligned}
$$

9.3. The remainding constructions and results are carried out parailel to the corresponaing ones for the family of the rational normal cubics on $X$ ( see $\S 7$ ).

$$
\text { (9.3.1) Let } z \text { and } \hat{\varphi}: o_{\eta}(-1) \longrightarrow \eta^{*}\left(S^{2} M^{*}\right)
$$
be as above. vie define :

$$
\begin{aligned}
& \tilde{D}_{k}=\tilde{D}_{k}(\hat{\varphi})=\left\{\left(z ; U_{k}\right)=\left(V_{4}, q ; U_{k}\right) \in G G_{Z}\left(k, M^{*}\right):\right. \\
& \left.: I m(q) \subseteq U_{k}\right\} \subset G_{Z}\left(k, M^{*}\right), 0 \leqslant k \leqslant 4 .
\end{aligned}
$$

(9.3.2) It is not hard to prove that the first determinantal $D_{1}(\hat{\varphi}) \subset z$ vanishes for the general intersection $X=G \cap P\left(V_{8}\right) \cap Q$, hence, the natural projection

$$
\pi_{2}: \tilde{D}_{2}(\hat{\varphi}) \longrightarrow D_{2}(\varphi)(\text { see }(7.1 .1)) \text { is an }
$$

isomorphism for the general X (see Remark (7.1.2)). 
(9.3.3) From Corollany (3.3.3) (2) we have that the curve $\quad C\left(P\left(\nabla_{4}\right)\right) \subset \mathbb{X}$ is a linearly normal curve of arithmetical genus 1 and of degree 4 ; the Iinear normality follows froil the fact that the curve $C\left(F\left(V_{4}\right)\right)$ is a component of the canonical curve $\mathrm{C}\left(\mathrm{P}\left(\mathrm{V}_{4}\right)\right)+\mathrm{C}_{I}$, $I=P\left(V_{2}\right)($ see $(3.3 .2)$ and Cor. $(3.3 .3))$, where $\nabla_{2} \in G(2, V)$ is choosen in such a way that $\nabla_{2}<\nabla_{4}$. In particular, in the case, when $C\left(P\left(V_{4}\right)\right)$ splits into a sum of two plane sections: $C\left(P\left(\mathrm{~V}_{4}\right)\right)=q+\bar{q}$, we obtain that $(q, \bar{q})=2$, hence, the intersection $\langle q \cap \bar{q}\rangle$ of the spaces $\langle q\rangle=\operatorname{span}(q)$ and $\langle\bar{q}\rangle=$ $=\operatorname{Span}(\bar{q})$ is a line in $P\left(\nabla_{8}\right)$. In the case when the two points of intersection of $q$ and $\bar{q}$ coincide, the line $\langle q \cap \bar{q}\rangle$ is equal, by definition, to their common tangent line ( see also (5.4.1) and (5.4.2)).

(9.3.4) The tauthological sequence on $G_{Z}(2, M)$, together with the injection $\hat{\varphi}: O_{\eta}(-1) \smile \eta^{*} s^{2} M^{*}$, define, as in $(7.2 .2)$, a section $s_{0}$ of the sheaf $O_{\eta}(1) \otimes \operatorname{Coker}(2)$ over $G_{Z}(2, M)$, where Coker $(2)=$ Coker $\left(s^{2} \tau_{2, N_{1}^{*}} \longrightarrow \eta^{*} s^{2} M^{*}\right)$.

(9.3.5) The same arguments, as in Corollary (7.2.4), give us a ground to impose the following open conditions on the subspace $\nabla_{8} \subset \nabla_{10}=\Lambda^{2} \nabla\left(\operatorname{dim}\left(\nabla_{8}\right)=8\right)$ and on the quadric $Q$, where $X=G \cap P\left(V_{8}\right) \cap Q$ ( see also Corollary (6.3.5) and Remark $(7 \cdot 1.2)$ ): 
(i) $X=G \cap P\left(V_{8}\right) \cap Q$ is smooth;

(ii) $\operatorname{dim}\left(v_{8} \cap\left(\Lambda^{2} \nabla_{4}\right)\right)=4$, for every $v_{4} \in P\left(V^{*}\right)=$ $=G(4, V)$;

(iii) $D_{1}(\hat{\varphi})=\varnothing$.

(9.3.6) CIAIN ( Logacher, $\left.\left[I_{1}\right]\right)$. Let $x=G \cap P\left(V_{8}\right) \cap 6$ where the subspace $\nabla_{8} \subset \Lambda^{2} \nabla$ and the quadric $Q$ fulfil the open conditions (i), (ii) and (iii). Let $Z, M$, $\hat{\varphi}, s_{0}$ and $F_{0}=F / 1$, where $F$ is the surface of conics on $X$ (see Definition $(4.4 .2)$ ), be as above. Then $F_{0}$ is embedded in $G_{2}\left(2, M^{*}\right)$ as a zero scheme of the section $s_{0}$, i.e.

$$
F_{0} \stackrel{\sim}{\longrightarrow}\left(s_{0}\right) \subset G_{Z}\left(2, M^{*}\right) \text { : }
$$

(9.3.7) The section $s_{0}$, together with the duality $G_{Z}\left(2, M^{*}\right) \simeq G_{Z}(2, M)$ define a section $s_{0}^{*}$ with zeros on $G_{Z}(2, M)$ ( see $\left.(7.2 .5)\right)$. So we can formulate (9.3.8) COROLIARY. In the conditions of the Claim (9.3.6) the"dual" natural embedding $F_{0} C_{Z}(2, M)$ represents the isomorphic image of $F_{a}$ in $G_{Z}(2, M)$ as a zero scheme of the section $s_{0}^{*}$, i.e.

$$
F_{0} \stackrel{\sim}{\longrightarrow}\left(\mathrm{s}_{0}^{*}\right) \subset G_{Z}(2, M) \text {. }
$$

Moreover, this embedding can be described as follows: Let $\left(q, v_{4}\right)$ and $\left(\bar{q}, v_{4}\right)=i\left(q, v_{4}\right)$ be a pair of involutive elements ( conics) of $F$. Let $\mathrm{H}_{q}$ and $\mathrm{H}_{\overline{\mathrm{q}}} \in \mathrm{M}^{*}\left(\mathrm{~V}_{4}\right)$ be the hyperplanes in $\mathrm{M}\left(\mathrm{V}_{4}\right)$, such that $\langle q\rangle=\operatorname{Span}(q)=P\left(H_{q}\right)$ and $\langle\bar{q}\rangle=\operatorname{Span}(\bar{q})=P\left(H_{\bar{q}}\right)$. 
Then the embedding $F_{0} C G_{Z}(2, M)$ sends the pair $\left(\left(q, v_{4}\right),\left(\bar{q}, \nabla_{4}\right)\right)$ to the triple $\left(V_{4}, H_{q} \cdot H_{\bar{q}} ; H_{q} \cap H_{\bar{q}}\right) \in G_{Z}(2, M)$. (9.3.9) RENARK. Note that $\mathrm{H}_{\mathrm{q}} \cap \mathrm{H}_{\overline{\mathrm{q}}}$ is always a plane since $P\left(\mathrm{H}_{\mathrm{q}} \cap \mathrm{H}_{\bar{q}}\right)$ coincides with the projective line $\langle q \cap \bar{q}\rangle \quad(\operatorname{see}(9.3 .3))$.

Similariy, as in the "rational normal cubic" case ( $\operatorname{see}(7.3 .1))$, the tauthological sequence on $\mathrm{P}_{Z}\left(M^{*}\right)$ together with the injection $\hat{\varphi}$ define a section $s$ of the sheaf $O_{\eta}(1) \otimes S^{2} \tau_{4, M}^{*}$ over $P_{Z}\left(M^{*}\right)$. The same arguments, as in $(7.3 .1)$ and $(7 \cdot 3.2)$, give

(9.3.10) CIAIM ( Iogachev, $\left[\mathrm{I}_{1}\right]$ ). In the conditions of the Claim (9.3.6), the family $F$ of the conics for the Fano threefold $X=x_{10}=G(2, V) \cap P\left(V_{8}\right) \cap Q$ is embedded naturally in the projective bundle $P_{Z}\left(M^{*}\right)$ as a zero scheme of the section $\mathbf{s}$, defined just above, that is:

$$
\mathrm{F} \longrightarrow \mathrm{Z}(\mathrm{s}) \subset \mathrm{P}_{\mathrm{Z}}\left(\mathrm{M}^{*}\right) \text {. }
$$

Using the duality $\mathrm{P}_{\mathrm{Z}}\left(\mathrm{M}^{*}\right) \simeq \mathrm{G}_{\mathrm{Z}}(3, \mathrm{M})$, we can formulate the following :

(9.3.11) COROLLARY. The embedding $F \hookrightarrow P_{Z}\left(M^{*}\right) \simeq G_{Z}\left(3, \mathbb{N}_{*}\right)$ sends the element $\left(q, \nabla_{4}\right) \in \mathbb{F}$ to the triple $\left(\mathrm{V}_{4}, \mathrm{H}_{\mathrm{q}} \cdot \mathrm{H}_{\overline{\mathrm{q}}} ; \mathrm{H}_{\mathrm{q}}\right) \in \mathrm{G}_{Z}(3, \mathrm{M})$ (the hyperplanes $\mathrm{H}_{\mathrm{q}}$ and $\mathrm{H}_{\overline{\mathrm{q}}}$ are defined as in the Corollary (9.3.8)) . 
9.4. Let now $\underline{P}_{F}: F \longrightarrow F_{0}=F / i$ be the two-sheeted covering, which conresponas to the involution $i: F \rightarrow P$ $(\sec (4.5 .1)$ and $(4.5 .2))$. The embedings $F_{0} \hookrightarrow G_{Z}(2, N)$ anò $F \hookrightarrow P_{Z}\left(\mathbb{N}^{*}\right)=G_{Z}(3, M)$, iogether witin $P_{F}$ jefine natural maps $F \rightarrow F_{0} \rightarrow G_{Z}(2, M)$ and $F \rightarrow G_{Z}\left(3, M_{1}\right)$. Therefore, the standard tauthological bundles $\tau_{2, M}$ on $G_{Z}(2, M)$ and $\tau_{3, M}$ on $G_{Z}(3, M)=P_{Z}\left(M^{*}\right)$ can be lifted on the family $F$. Just as in the "rational normal cubic" case, there is a representation of the tangent bundle $T_{F}$ by means of some bundle on $F$, which can be described in geometrical terms.

(9.4.1) CIAIM ( Logachev) The open Tangent Bundle Theorem for the family. $F$ of "conics" for $X$; see $\left[L_{1}\right]$ ). Let $I_{\sigma}=\left\{\left(q, V_{4}\right): q\right.$ is a $\sigma$-conic on $\left.x\right\} \subset F$ be the smooth rational curve of the $\sigma$-conics in the family of conics $F($ see $\S 4.2)$; let also $Z, M$, $G_{Z}(2, M), P_{Z}\left(M^{*}\right)$ and the bundles $\tau_{2, M}$ and $\tau_{3, M}$ be as abcre. Let $\mathscr{T}_{F}$ be the cotangent sheaf ( resp., the cotangent bundle) of $\mathbb{P}$.

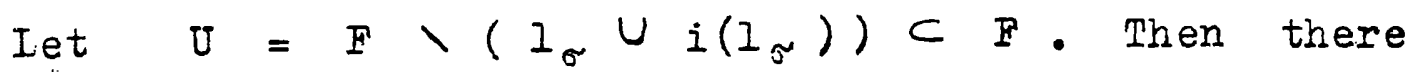
exists a naturally defined isomorphism of sheaves over the oper subset $\quad \cup F$ :

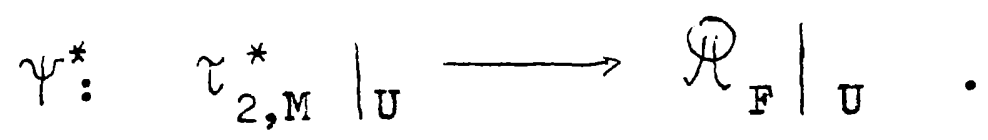

(9.4.2) REMARK. The curve $i\left(I_{\sigma}\right)$ coincides with the 
exceptional curve $r_{F}^{-1}\left(q_{0}\right)$ ( the curve of the exceptional "conics" of $F$ ( see $\S 4.2$, or the comments (9.2.2)). The curves $I_{\sigma}$ and $i_{(} I_{\sigma}$ ) are mutually disjoint (ibid.j Moreover, there is an exact global version of the T.B.T. for the family of conics, proven by Logachev:

(9.4.3) THEOREM ( Logachev, [ $\left.\mathrm{I}_{1}\right]$ ). There is a naturally definea exact sequence of sheaves over the surface of conics F for $\mathrm{X}$ :

$0 \longrightarrow \tau_{2, M}^{*} \longrightarrow r^{*} \mathscr{H}_{I_{\mathrm{m}}} \longrightarrow 0_{I_{\sigma}} \vee 0_{i\left(I_{\sigma}\right)} \longrightarrow 0$

where $r: F \longrightarrow F_{m}$ is the morphism onto tine minimal model $F_{m}$ of $F$. In fact, the morphism $r$ is a composition of $r_{F}: F \longrightarrow F_{C}$ (which blows-down the curve $r_{F}^{-1}\left(q_{0}\right)$ of the exceptional conics in $F$ ), and of the blowing-down of the $r_{F}$-image of its involutive curve (the curve of the $\sigma$-conics on $X$ in $\left.F_{c}\right)$. The both contractions blow-down (-1)-curves to (non-singular) points of the smooth minimal surface $F_{\mathrm{m}}$ ( see also $[\mathrm{P}]$ ).

(9.4.4) COMMENTS.

(1) Note that the statement of the global T.B.T. (9.4.3) is, in fact, a correct treatment of the analogue of the sequence $\left(1^{\prime \prime \prime}, l e\right)$ for the family $F$ (see (8.2.9), seq. $\left(1^{\prime \prime \prime}, \epsilon\right)$ and Remark $\left.(8.2 \cdot 10)\right)$.

(2) The factor-sheaf $O_{I_{\sigma}} \vee O_{i\left(I_{\sigma}\right)}$ has a support on the closet subset $I_{\sigma} U i\left(I_{\sigma}\right)$ of codim. $=1$ in $F$. (9.4.5) COROIIARY ( see $(8.2 .12))$. Iet

$$
\psi^{*}:\left.\left.T_{F}\right|_{U} \longrightarrow \tau_{2, M}\right|_{U} \text { be the }
$$


dual of $\Psi$ (see $\operatorname{claim}(9.4 .1))$. Then $\Psi^{*}$ coincides with the natural map

$$
\psi^{*}\left(q, V_{4}\right): T_{F}\left(q, V_{4}\right) \longrightarrow H_{q} \cap H_{\bar{q}} \subset M\left(V_{4}\right) \subset V_{8}
$$

on the fiber $T_{F}\left(c_{1}, v_{4}\right)$, where $\left(q, v_{4}\right) \in U$ and the hyperplanes $\mathrm{H}_{\mathrm{q}}$ and $\mathrm{H}_{\overline{\mathrm{q}}}$ are defined as in Corollary $(9.3 .8)$.

In the end I would like to express my gratitude to prof. V. A. Iskovskikh, V. Kaner and K. Ranestad for lending their comprehensive support. 


\section{REFERENCES}

[B1] A. Beauville, Les singularités du aiviseur tineta de la Jacobienne intermédiaire de l'hypersurface cubique dans $\mathrm{P}^{4}$, Iect. Notes in Nath. 947 , Springer-Verlag (1982), 190-208.

[B2] A. Beauville, Variétés de Prym et jacobienne intermédiaires, Ann. de I'ENS $4^{\text {eme }}$ série 10 (1977), 309-391.

[B3] Complex AIgebraic Surfaces, Iondon Niath. Soc. Lect. Note Series : 68 , Cambriage Univ. Press, Cambridge (1983).

[C] C. H. Clemens, Double Solids, S.dv. in Math. $47: 2$ (1983), , 107-230.

[CG] C. H. Clemens \& Ph. A. Griffiths, The intermediate Jacobian of the cubic threefold. Ann. of Math. 95 (1972), $281-356$.

[D] 0. Debarre, Le théoreme de Torelii pour les intersections de trois quadriques, Invent. Nath. 95: 3 (1989), 507-528.

[F] W. Fulton, Intersection Theory, Ergebn. der Math. und ihrer Grenzgebiete : 3 Folge. Band 2, A Series of Modern. Surv. in Math., Springer-Verlag, BerlinHeidelberg (1984).

[F1] G. Fano, Sulle varietà a tre dimensioni a curve-sezioni canoniche, Mem. R. Acad. d'Italia 8 (1937), $27-37$ 
[F2] G. Fano, Su alcune varietà algebriche a tre dimensioni razionali e aventi curve-sezioni canoniche, Comm. Math. Helvetici , 14 (1942), 202-211.

[FN] M. Furushima \& N. Nakayama, The family of lines on the Fano threefold $V_{5}$, Nagoya Math.J.,116(1989), 111-122.

[G] N.P. Gushel', On Fano varieties of genus 6 , Izv. A.N. SSSR Ser.Mat. 46:6(1982), 1.159-1174 (russian); English Transl, in Niath. USSR Izrestija 21 (1983), 445-459.

[GH] Ph.A. Griffiths \& J. Harris, Principles of Algebraic Geometry, John Willey \& Sons, New York (1978).

[H] S,I. Hashin, Birational automorphisms of the double Veronese cone of dimension three, Vest.Mosk.Univ. Ser.I, No 1 (1984), 13-16 (russian); English Transl. in Moscow Univ. Math. Bull. 39:1 (1984)

[I1] V.A. Iskorskikh, Fano threefolds I, Izv. A.N. SSSR Ser. Mat. 41:3 (1977) , 516-562 (russian) ; English Transl. in Math. USSR Izvestija 11:3 (1977), 485-527.

[I2] V.A. Iskovskikh, Pano threefolds II, Izv. A.N. SSSR Ser. Mat. 42:3 (1978) , 506-549 (russian); English Transl. in Math. USSR Izvestija 12:3 (1978), 469-506.

[I3] V.A. Iskovskikh, Birational automorphisms of three-dimensional algebraic varieties, Itogi Nauki i Tekhniki: Sovr. Probl. Mat. - Vol. 12 , VINITI, Moseow (1979), 159-236 (russian); English Transl. in Journ. Soviet Math. Vol. 13 No 6 (1980), 815-868. 
[I4] V. A. Iskovskikh, Lectures on algebraic tinreefolàs: Fano threefolds, Noscow Univ. Publ., Noscow (1988).

[IM] V. A. Iskovskikh \& Yu. I. Nanin, Three-dimensionai quartics and counterexamples to the Iüroth problem, Nat. Sbornik 86 (1971), 140-166 (russian); English Transl. in Nath. USSR Sb. 15 (1971), 14t-166.

[I] M. Letizia, The Abel-Jacobi mapping for the quartic threefold, Invent. Math. 75 (1984), 477-492.

[I1] Dm. Yu. Logachev, Abel-Jacobi isogeny for the Fano threefold of genus 6 , Yaroslavi' Gos. Ped. Inst. K. D. Ushinskij (1982) - Thesis (russian).

[I2] Im. Yu. Iogachev, Abel-Jacoti isogeny for the Fano threefola of genus 6 , sb. Constructive Algebraic Geometry, Yaroslavl' , Vol. 200 (1982) , 67-76.

[M] Dm. G. Narkushevich, Numerical invariants of the families of lines of some Fano varieties, Nat. Sbornik $116: 2$ (1981) , 265-288 (russian); English Transl, in Mati. USSR Sb. 45 (1981)

[MM1] S. Mori \& S. Mukai, Classification of Fano threefolds with $B_{2} \geqslant 2$, I - Algebraic and Topological Theories, Tokyo and Amsterdam : Kinokuniya Company ITD (1985), 496-548.

[MM2] S. Mori \& S. Mukai, Classification of Fano threefolds with $B_{2} \geqslant 2$, Manuscr. Niath. $36: 2$ (1981), 147-162.

[P] P.J. Puts, on some Fano threefolds that are sections of Grassmannians, Indagationes Math. 44 (1982), 77-90. 
[R] I. Rotin, Sulle $V_{3}$ algebriche su cui l'aggiunzione si estingue, Rend. Acc. Iincei(8) 9 (1950), 246-249.

[T1] Al. S. Tikhomirov, Geometry of the Fano surface of the double space of $\mathrm{F}^{3}$ with ramification on a quartic, Izv. A.N. SSSR Ser. Mat. 44:2 (1980), 415-442 (russian); English Transl. in Math. USSR Izv. 16 (1981)

[T2] AI. S. Tikhomirov, The Abei-Jacobi map of sextics of genus 3 on double spaces of $P^{3}$ of index two, Dokl. A.N. SSSR 286:4 (1986), 82i-824 (russian); English Transl. in Soviet Math. Dokl. $33: 1$ (1986), 204-206.

[T3] Al. S. Tikhomirov, The Fano surface of a double Veronese cone, Izv. A.N. SSSR Ser. Mat. 45:5 (1981), 1121-1197 (russian) ; Englisi Transl. in Math. USSR Izv. 19 (1982)

[TN] A. N. Tjurin, on the intersection of quadrics - I, II, Usp. Mat. Nauk $30: 6$ (1975) , 51-99 (russian) ;English Transl. in Russian Math. Surveys 30:6(1975), 51-105.

[V] C. Voisin, Sur la jacobienne intermédiaire du double solide d'indice deux, Duke Math. Journal $57: 2$ (1988), 629-646.

[W] G. E. Welters, Abel-Jacobi isogenies for certain types of Fano threefolds, Math. Centre Tracts 141 , Math. Centrum, Amsterdam (1981) - The'sis. 
